Victoria Serhiyenko, Alexandr Serhiyenko

\title{
DIABETIC CARDIOVASCULAR NEUROPATHY
}


UDK 616.379-008.64

BBK 54.15

S 49

\section{Consultant:}

Man'kovskiyBN., Professor, Corresponding Member of the NAMS, Head of the Diabetology Department of the P.L. Shupik National Medical Academy of Postgraduate Education, Kyiv, Ukraine

\section{S 49}

\section{Victoria Serhiyenko, Alexandr Serhiyenko}

Diabetic cardiovascular neuropathy. Stavropol: Logos Publishers, 2018. $111 \mathrm{p}$.

ISBN 978-5-905519-86-4

DOI: $10.18411 /$ dia012018.49

Cardiac autonomic neuropathy (CAN) is a serious complication of diabetes mellitus, that is strongly associated with increased risk of cardiovascular mortality. Although it is common complication, the significance of CAN has not been fully appreciated and there are no unified treatment algorithms for today. In this review we have analyzed the existing data about the known risk factors, screening and diagnostic algorithm, staging of CAN and possible treatment, including effectiveness of lifestyle modification, intensive glycemic control; treatment of diabetic dyslipidemia; antioxidants; vitamins; correction of vascular endothelial dysfunction; prevention and treatment of thrombosis; treatment of orthostatic hypotension. 


\section{CONTENTS}

\begin{tabular}{|c|c|c|}
\hline & About the authors & 7 \\
\hline & Preface & 8 \\
\hline & Introduction & 9 \\
\hline 1. & $\begin{array}{l}\text { Risk factors for the diabetic cardiovascular autonomic } \\
\text { neuropathy }\end{array}$ & 10 \\
\hline 2. & $\begin{array}{l}\text { Morbidity and mortality in diabetic cardiovascular autonomic } \\
\text { neuropathy }\end{array}$ & 12 \\
\hline 3. & Classification of diabetic autonomic neuropathies & 13 \\
\hline 3.1. & Classification of diabetic cardiovascular autonomic neuropathy & 14 \\
\hline 4. & Screening and diagnosis & 14 \\
\hline 5. & $\begin{array}{l}\text { Clinical impact of diabetic cardiovascular autonomic } \\
\text { neuropathy }\end{array}$ & 15 \\
\hline 5.1. & Clinical manifestations of cardiovascular autonomic neuropathy & 15 \\
\hline 6. & Cardiovascular autonomic neuropathy assessment & 17 \\
\hline 6.1. & Assessment of symptoms & 17 \\
\hline 6.2 . & Assessment of signs & 17 \\
\hline 6.2.1. & Resting tachycardia & 17 \\
\hline 6.2.2. & Exercise intolerance & 17 \\
\hline 6.2.3. & Orthostatic hypotension & 18 \\
\hline 6.2.4. & Orthostatic tachycardia and bradycardia syndromes & 18 \\
\hline 6.2.5. & QTi prolongation & 19 \\
\hline 6.2.6. & Impaired heart rate variability & 19 \\
\hline 6.2.7. & Non-dipping and reverse dipping & 20 \\
\hline 6.2.8. & "Silent" myocardial ischemia/cardiac denervation dyndrome & 20 \\
\hline 6.3. & Cardiovascular autonomic neuropathy and sudden death & 21 \\
\hline 6.4. & Intraoperative cardiovascular lability & 21 \\
\hline 7. & Diagnostic testing for cardiovascular autonomic neuropathy & 23 \\
\hline 7.1. & Cardiovascular autonomic reflex tests & 23 \\
\hline 7.2. & Short-term ECG & 26 \\
\hline 7.3. & Heart rate variability & 26 \\
\hline 7.4. & Heart rate turbulence & 28 \\
\hline 7.5. & Baroreflex sensitivity & 28 \\
\hline
\end{tabular}


Victoria Serhiyenko, Alexandr Serhiyenko

\begin{tabular}{|c|c|c|}
\hline 7.6. & Muscle sympathetic nerve activity & 29 \\
\hline 7.7 . & Catecholamine assessment and cardiovascular sympathetic tests & 30 \\
\hline 7.8. & Heart sympathetic imaging and heart function tests & 31 \\
\hline 7.9 . & Diagnostic testing for orthostatic symptoms & 33 \\
\hline 7.10 & $\begin{array}{l}\text { Diagnostic algorithm for diabetic cardiovascular autonomic } \\
\text { neuropathy }\end{array}$ & 33 \\
\hline 7.11 & Differential diagnosis of diabetic neuropathies & 33 \\
\hline 7.12. & Neuropathy end points for research and clinical practice & 33 \\
\hline 7.13. & Diagnostic criteria for cardiovascular autonomic neuropathy & 34 \\
\hline 7.14 & Staging of cardiovascular autonomic neuropathy & 36 \\
\hline 8. & $\begin{array}{l}\text { Prevention of the diabetic cardiovascular autonomic } \\
\text { neuropathy }\end{array}$ & 37 \\
\hline 9. & $\begin{array}{l}\text { Treatment of the diabetic cardiovascular autonomic } \\
\text { neuropathy }\end{array}$ & 38 \\
\hline 9.1. & Intensive glucose control & 38 \\
\hline 9.2 . & Lifestyle modifications & 38 \\
\hline 9.3 . & Glucose control & 40 \\
\hline 9.4 . & Drugs for treatment of hypercholesterolemia & 42 \\
\hline 9.4.1. & $\begin{array}{l}\text { The 3-hydroxy-3-methylglutaryl-coenzyme (HMG-CoA) } \\
\text { reductase inhibitors }\end{array}$ & 42 \\
\hline 9.4.2. & Cholesterol absorbion (ezetimibe) & 45 \\
\hline 9.4.3. & $\begin{array}{l}\text { PCSK9 inhibitors (Proprotein convertase subtilisin/kexin type 9) } \\
\text { inhibitors (alirocumab, evolocumab) }\end{array}$ & 45 \\
\hline 9.4.4. & Fibric acid derivatives (gemfibrozil, fenofibrate, fenofibric acid) & 47 \\
\hline 9.4.5. & Niacin (nicotinic acid) & 48 \\
\hline 9.4.6. & Bile acid sequestrants & 49 \\
\hline 9.4.7. & Inhibitors of microsomal triglycerides transfer protein & 51 \\
\hline 9.4 .8 & $\begin{array}{l}\text { Antisense apolipoprotein B oligonucleotide (mipomersen via } \\
\text { subQ injection) }\end{array}$ & 52 \\
\hline 9.4.9. & Omega-3 fatty acids & 53 \\
\hline 9.5 . & $\begin{array}{l}\text { Specific features of dyslipidaemia in insulin resistance and type } 2 \\
\text { diabetes mellitus }\end{array}$ & 56 \\
\hline 9.6. & Evidence for low-density lipoprotein-lowering therapy & 56 \\
\hline 9.7 . & Triglycerides and high-density lipoprotein cholesterol & 56 \\
\hline 9.8 . & $\begin{array}{l}\text { Treatment strategies for patients with type } 2 \text { diabetes mellitus and } \\
\text { metabolic syndrome }\end{array}$ & 57 \\
\hline
\end{tabular}




\begin{tabular}{|c|c|c|}
\hline 9.9 . & $\begin{array}{l}\text { Fatty acids metabolism disorders ( } \gamma \text {-linolenic acid, acetyl } \\
\text { L-carnitine) }\end{array}$ & 57 \\
\hline 9.10 & Antioxidant therapy & 58 \\
\hline 9.10 .1 & $\begin{array}{l}\text { Strategies targeted directly against reactive oxygen species and } \\
\text { reactive nitrogen species }\end{array}$ & 59 \\
\hline 9.10.1.1. & Alpha-lipoic acid & 59 \\
\hline 9.10.1.2. & Vitamins $\mathrm{A}, \mathrm{B}_{1}, \mathrm{~B}_{3}, \mathrm{C}$ and $\mathrm{E}$ & 60 \\
\hline 9.10.1.2.1. & Vitamin A & 60 \\
\hline 9.10.1.2.2. & Vitamin $\mathrm{B}_{1}$ & 60 \\
\hline 9.10.1.2.3. & Nicotinamide (Vitamin $\mathrm{B}_{3}$ ) & 61 \\
\hline 9.10.1.2.4. & Vitamin C & 61 \\
\hline 9.10.1.2.5. & Vitamin E & 61 \\
\hline 9.10.2. & Strategies targeted against individual oxidative stress pathways & 64 \\
\hline 9.10.2.1. & Aldose reductase inhibitors & 64 \\
\hline 9.10.2.2. & Protein kinase $\mathrm{C}$ inhibitors & 65 \\
\hline 9.10 .3 & Anti-advanced glucose end-products agents & 65 \\
\hline 9.10.3.1. & Benfotiamine & 65 \\
\hline 9.10.3.2. & Aminoguanidine & 78 \\
\hline 9.10 .4 & Aspirin & 78 \\
\hline 9.10 .5 & Strategies targeted at mitochondria & 80 \\
\hline 9.10.5.1. & Coenzyme Q & 80 \\
\hline 9.10.5.2. & Telmisartan & 81 \\
\hline 9.10.5.3. & Metformin & 81 \\
\hline 9.10.5.4. & Pioglitazone & 82 \\
\hline 9.10.6. & Triple antioxidant therapy & 82 \\
\hline 9.11. & Correction of vascular endothelial dysfunction & 82 \\
\hline 9.11.1. & Trimetazidine & 82 \\
\hline 9.11.2. & Perhexiline & 83 \\
\hline 9.11.3. & Ranolazine & 83 \\
\hline 9.12. & Beta blockers & 83 \\
\hline 9.13. & Dihydropyridine calcium channel blockers & 84 \\
\hline 9.14. & Phosphodiesterase-5 inhibitors & 84 \\
\hline
\end{tabular}




\begin{tabular}{|c|l|c|}
\hline 9.15. & Ivabradine, the cardiac pacemaker "funny" $\left.\left(\mathrm{I}_{\mathrm{f}}\right)\right)$ inhibitor & 85 \\
\hline 9.16. & Thrombosis prevention and treatment & 85 \\
\hline 9.17. & Treatment of orthostatic hypotension & 86 \\
\hline & Conclusion & 88 \\
\hline & References & 89 \\
\hline & Abbreviations list & 108 \\
\hline
\end{tabular}




\section{ABOUT THE AUTHORS}

Victoria A Serhiyenko, MD, PhD in Medicine Sciences, DMSc, Associate Professor of the Department of Endocrinology, Lviv National Medical University named by Danylo Halitsky, Lviv, Ukraine. At the moment she is working on the problems concerted with the revelation of the correlating connections between activities of some metabolic, hormonal and other parameter, and state of the proand anti-inflammatory factors in blood in patients with diabetes mellitus which depends on the character of diabetic complications. The second part of his scientific work is the research of the effectively of the benfotiamine in treatment and prevention of diabetic complications in clinical practice.

Victoria Serhiyenko has published 238 scientific papers. These works are devoted to the problems of pathogenesis, diagnosis and treatment, prevention of the diabetic cardiovascular autonomic neuropathy.

Alexandr A Serhiyenko, MD, PhD in Medicine Sciences, DMSc, Professor of the Department of Endocrinology, Lviv National Medical University named by Danylo Halitsky, Lviv, Ukraine. At the moment he is working on the problems concerted with the revelation of the correlating connections between activities of some metabolic parameters in patients with diabetes mellitus which depends on the character of diabetic complications. The second part of her scientific work is the research of the effectively of the benfotiamine in treatment and prevention of diabetic complications in clinical practice.

Alexandr Serhiyenko has published 613 scientific papers. These works are devoted to the problems of pathogenesis, diagnosis and treatment, prevention of the diabetic complications. 


\section{PREFACE}

Cardiovascular autonomic neuropathy (CAN) is a serious complication of diabetes mellitus (DM), that is strongly associated with approximately five-fold increased risk of cardiovascular mortality. CAN manifests in a spectrum of things, ranging from resting tachycardia and fixed heart rate to development of "silent" myocardial infarction. Clinical correlates or risk markers for CAN are age, DM duration, glycemic control, hypertension, and dyslipidemia (DLP), development of other microvascular complications. Established risk factors for CAN are poor glycemic control in type $1 \mathrm{DM}$ and a combination of hypertension, DLP, obesity, and unsatisfactory glycemic control in type $2 \mathrm{DM}$. Symptomatic manifestations of CAN include sinus tachycardia, exercise intolerance, orthostatic hypotension $(\mathrm{OH})$, abnormal blood pressure (BP) regulation, dizziness, presyncope and syncope, intraoperative cardiovascular instability, asymptomatic myocardial ischemia and infarction. Methods of CAN assessment in clinical practice include assessment of symptoms and signs, cardiovascular reflex tests based on heart rate (HR) and BP, short-term electrocardiography (ECG), QT interval prolongation, HR variability (24-h, classic $24 \mathrm{~h}$ Holter ECG), ambulatory BP monitoring, HR turbulence, baroreflex sensitivity, muscle sympathetic nerve activity, catecholamine assessment and cardiovascular sympathetic tests, heart sympathetic imaging. Although it is common complication, the significance of CAN has not been fully appreciated and there are no unified treatment algorithms for today. Treatment is based on early diagnosis, life style changes, optimization of glycemic control and management of cardiovascular risk factors. Pathogenetic treatment of CAN includes: balanced diet and physical activity; optimization of glycemic control; treatment of DLP; antioxidants, first of all $\alpha$-lipoic acid (ALA), aldose reductase inhibitors, acetyl-Lcarnitine; vitamins, first of all fat-soluble vitamin B1; correction of vascular endothelial dysfunction; prevention and treatment of thrombosis; in severe casestreatment of orthostatic hypotension. The promising methods include prescription of prostacyclin analogues, thromboxane $\mathrm{A}_{2}$ blockers and drugs that contribute into strengthening and/or normalization of $\mathrm{Na}^{+}, \mathrm{K}^{+}$-ATPase (phosphodiesterase inhibitor), ALA, dihomo- $\gamma$-linolenic acid (DGLA), $\omega-3$ polyunsaturated fatty acids ( $\omega-3$ PUFAs), and the simultaneous prescription of ALA, $\omega-3$ PUFAs and DGLA, but the future investigations are needed. Development of $\mathrm{OH}$ is associated with severe or advanced CAN and prescription of nonpharmacological and pharmacological, in the foreground midodrine and fludrocortisone acetate, treatment methods are necessary. 


\section{INTRODUCTION}

The number of people with type 2 diabetes mellitus (T2DM) has been increasing worldwide. It was estimated that there were 415 million people with diabetes aged 20-79 years in 2015, and the number was predicted to rise to 642 million by 2040 [1].

The majority of patients with long-term course of diabetes mellitus (DM) (mainly T2DM) are diagnosed with coronary heart disease (CHD) due to coronary vessels arterial sclerotic disease. Often the course of CHD is complicated by combination of hypertension, specific kidney arterial involvement, eyes and lower limbs affection and metabolic alterations in the myocardium that are combined with early coronary atherosclerosis.

The term "diabetic heart or diabetic cardiomyopathy" is associated with coronary vessels affection, myocardium changes, diabetic cardiovascular autonomic neuropathy (CAN) and arterial sclerotic disease among middle age and elderly patients with prolonged duration of DM. Conditionally, there are two main forms of heart disease in case of DM: diabetic cardiomyopathy (non-coronary genesis) and ischemic heart disease. There is a metabolic stage (actual cardiomyopathy); metabolic-ischemic stage-ischemic heart disease; myocardial infarction (MI); dystrophic coronary cardiosclerosis; CAN [2, 3].

Cardiac autonomic neuropathy among T2DM patients, is characterized by lesion of nerve fibers in the sympathetic and parasympathetic divisions of the autonomic nervous system (ANS), is diagnosed unsatisfactorily and may be accompanied by severe ortosthatic hypotension $(\mathrm{OH})$, decreased tolerance to the physical loadings, and cause of the cardiac arrhythmias, ischemia of coronary vessels, "silent" MI, sudden death syndrome $[4,5,6]$. CAN, especially at the early stages, can be subclinical and thus as the disease progresses, it becomes clinically evident $[7,8,9]$.

Based on the CAN Subcommittee of the Toronto Consensus Panel on Diabetic Neuropathy [5] and the American Diabetes Association (ADA) [3], CAN is defined as the impairment of cardiovascular autonomic control among patients with established DM following the exclusion of other causes. CAN is usually documented by using several cardiovascular autonomic reflex tests (CARTs) $[5,10]$.

Therefore, the problem of effective diagnosis and treatment of CAN is particularly relevant. Pathogenetic treatment of CAN includes: balanced diet and physical activity; reducing insulin resistance (IR); intensive glycemic control; treatment of diabetic dyslipidemia (DLP); antioxidants; vitamins; correction of metabolic abnormalities in myocardium; prevention and treatment of thrombosis; treatment of $\mathrm{OH}$; symptomatic treatment of concomitant diseases and syndromes (hypertension, CHD, heart failure and arrhythmias) and others [11-19]. The aim of this book is to review the latest evidence and own data about the risk factors, prophylaxis, early diagnosis, treatment, and treatment perspectives of patients with $\mathrm{DM}$ and CAN. 


\section{RISK FACTORS FOR THE DIABETIC CARDIOVASCULAR AUTONOMIC NEUROPATHY}

The risk of developing autonomic dysfunction in DM depends on several factors. However, two of them are common to both type 1 DM (T1DM) and T2DM: duration of disease and the degree of glycemic control. In both DM types, poor blood glucose control plays an important role both in the initial pathophysiology [oxidative stress (OS), microcirculation dysfunction due to nitric oxide (NO) loss and Schwann cell lesion due to accumulation of free radicals] as well as in its progression (axonal degeneration and neuronal apoptosis) [20, 21].

Although diabetic neuropathies are classified among DM microangiopathic complications, the pathophysiological mechanism is multifactorial, and there is enough evidence that small-fiber diabetic polyneuropathy (DPN) and even CAN may precede DM [peripheral neuropathies (PNP)] related to glucose intolerance or prediabetes) [22]. The presence of CAN, however, is not only associated with the duration and degree of hyperglycemia. Several studies consistently showed that cardiovascular risk factors, such as systolic blood pressure (BP), body mass index (BMI), triglycerides (TGs), and smoking, play an important role in the development of CAN. It is noteworthy that these factors share at least two points in common: they are potentially modifiable and are associated with IR, once again suggesting that CAN may arise with a metabolic syndrome [21].

Even more important, however, were the results of the Intensified Multifactorial Intervension in Patients With Type 2 Diabetes and Microalbuminunia (Steno 2) study, in which the intensified multifactorial intervention (hyperglycemia, hypertension, DLP and microalbuminuria) in patients with T2DM reduced the risk of CAN progression by $68 \%[23,24]$. The role of intensive control in preventing and slowing the progression of CAN in patients with T1DM is also well-known: in the classical the Diabetes Control and Complications Trial (DCCT), its prevalence was reduced by $53 \%[21,25]$.

The main predictors for the development of CAN in patients with T2DM are age, gender, ethnicity and presence of microvascular complications (nephropathy, retinopathy, and PNP) [6]. In a cohort of 1.000 patients with T2DM, the incidence of CAN 7.5 years of follow-up was correlated with older age and the presence of microvascular disease [26]. In terms of gender, in a multicenter study of 3.250 patients with DM, there was no difference in the prevalence of CAN between men and women (men 35\% and women 37\%) [27]. However, the Action to Control Cardiovascular Risk in Diabetes (ACCORD) study which involved more than 8.000 T2DM patients, CAN was more prevalent in women $(2.6 \%$ in men and $4.7 \%$ in women for moderate to severe and $1.4 \%$ in men and $2.2 \%$ in women for severe) [28].

In addition to age and diabetes duration, other diabetes-related clinical correlates or predictors of CAN (as documented in cross-sectional or longitudinal studies) were glycemic control, the presence of DPN, diabetic retinopathy, microalbuminuria or diabetic nephropathy, and renal failure $[5,19,22,29,30]$. The role of several cardiovascular risk factors has also been increasingly reported: BP or hypertension, smoking (only in cross-sectional studies), low-density lipoprotein cholesterol (LDL-C), high-density lipoprotein cholesterol (HDL-C), TGs, body weight or obesity in T2DM (with some controversy), waist circumference, insulin levels in T2DM, 
cardiovascular disease, and use of anti-hypertensive drugs [5, 19, 29-31].

Current data that differentiate CAN in T1DM and in T2DM in terms of risk factors and natural history are summarized in Table 1 [21].

Table 1 Cardiovascular autonomic neuropathy in Type 1 and Type 2 diabetes mellitus: differences in relation to risk factors and natural history [21]

\begin{tabular}{|c|c|c|}
\hline \multicolumn{3}{|c|}{ Diabetes mellitus } \\
\hline Risk factors & $\begin{array}{c}\text { Type } 1 \text { diabetes } \\
\text { mellitus }\end{array}$ & Type 2 diabetes mellitus \\
\hline Age & + & + \\
\hline Gender (female) & + & - \\
\hline Obesity & - & + \\
\hline Hyperinsulinemia & NA & + \\
\hline Duration of DM & ++ & ++ \\
\hline Smoking & + & + \\
\hline $\mathrm{HbA}_{1 \mathrm{c}}$ & ++ & ++ \\
\hline Hypertension & ++ & + \\
\hline Retinopathy & ++ & + \\
\hline Hypertriglyceridemia & + & + \\
\hline Classical DPN & ++ & ++ \\
\hline Microalbuminuria & ++ & ++ \\
\hline $\begin{array}{l}\text { Dyslipoproteinemia ( }>\text { LDL and }< \\
\text { HDL }\end{array}$ & + & $(+)$ \\
\hline Prevalence at diagnosis of DM & $7.7 \%$ & $5 \%$ \\
\hline Prevalence after 10 years & $38 \%$ & $65 \%$ \\
\hline Prevalence (random) & $25 \%$ & $34 \%$ \\
\hline
\end{tabular}

++ Strong association; + - Moderate association; - Not found; (+) Controversial; NA - Not applicable.

Possible factors associated with high mortality and sudden death due to autonomic neuropathy are [27]:

- silent myocardial ischaemia (SMI)/infarction;

- cardiorespiratory arrest/increased perioperative and peri-intubation risk;

- resting tachycardia;

- ventricular arrhythmias/prolongation of the QT interval (QTi);

- hypertension;

- orthostatic hypotension;

- flattening of the nocturnal reduction of BP and heart rate ("non-dipper" phenomenon); 
- exaggerated BP responses with supine position and exercise;

- abnormal diastolic/systolic left ventricular function;

- poor exercise tolerance;

- impaired cardiovascular responsiveness;

- heat intolerance due to defective sympathetic thermoregulation;

- susceptibility to foot ulcers and amputations due to arteriovenous shunting and sudomotor dysfunction;

- hypoglycaemia unawareness;

- increased risk of severe hypoglycaemia;

- obstructive sleep apnoea syndrome.

\section{MORBIDITY AND MORTALITY IN DIABETIC CARDIOVASCULAR AUTONOMIC NEUROPATHY}

CAN is a significant cause of morbidity and mortality associated with a high risk of cardiac arrhythmias and sudden death, possibly related to SMI. Cardiovascular disease remains the main cause of excess mortality among patients with T1DM and T2DM. Reduced heart rate variability (HRV) as a marker of autonomic dysfunction has been shown to have dire consequences in terms of morbidity (e.g. progression of coronary atherosclerosis) and mortality independent of cardiovascular risk factors in various populations, including those with prediabetes and DM [32]. In T1DM patients, there is a fourfold increase risk of death [33,34]. CAN is significantly associated with overall mortality $[5,35,36]$, and in some but not all studies with morbidity, such as SMI, coronary artery disease (CAD), stroke, diabetic nephropathy progression and perioperative morbidity. In the Detection of Ischemia in Asymptomatic Diabetic Subjects (DIAD) study, a diminished Valsalva heart rate (HR) ratio (a measure of CAN) was strongly associated with SMI, independent of more traditional risk factors including sex, age, hypertension and smoking [5,37]. In the European Epidemiology and Prevention of Diabetes (EURODIAB) study, autonomic dysfunction was present in one-third of T1DM patients, and was strongly associated with coexisting cardiovascular disease (CVD) after adjustment to age, glycated hemoglobin $\left(\mathrm{HbA}_{1 \mathrm{c}}\right)$ and duration of diabetes [32]. Results from the ACCORD trial again confirmed the association of CAN and mortality. These investigations showed that the individuals in this trial with baseline CAN were 1.55-2.14 times as likely to die as individuals without CAN [5, 28]. Furthermore, CAN in the presence of DPN was the highest predictor of CVD mortality. Indeed, combining indexes of autonomic dysfunction have been shown to be associated with the higher risk of mortality $[5,34,35]$. There is also strong evidence, based on studies in patients with T1DM and patients with T2DM with a mean follow-up of 9.2 years, that QTi prolongation is an independent predictor of mortality for all-cause and cardiovascular deaths [5, 8, 34, 35, 38]. Thus, CAN assessment can be used for cardiovascular risk stratification in patients with and without established $\mathrm{CVD}$; as a marker for patients requiring more intensive monitoring during the perioperative period and other physiological stresses; and as an indicator for more intensive pharmacotherapeutic and lifestyle management of comorbid conditions. 
There is definitive evidence for a predictive value of CAN on overall mortality (class I) and some evidence of a predictive value of CAN on morbidity (class II).

$\mathrm{OH}$, when due to advanced $\mathrm{CAN}$, is associated with an additional increase in mortality risk over that driven by HRV abnormalities (class III).

Some cardiovascular abnormalities, closely linked to CAN, are associated with increased mortality: tachycardia (class II), QTi prolongation (class II), and nondipping status (class III) [5].

CAN is a risk marker of mortality (level A) as well as a risk marker and likely a risk factor for cardiovascular morbidity (level B), and possibly a progression promoter of diabetic nephropathy (level C).

C).

$\mathrm{OH}$ is associated with a worse prognosis than cardiovagal neuropathy (level

QTi prolongation has prognostic value in diabetes (level B).

Non-dipping status is associated with an adverse cardiovascular prognosis in diabetes (level C). Non-dipping status predicts the progression from micro- and macroalbuminuria to renal failure in T2DM (level C) [5].

Conclusions. The prevalence of confirmed CAN in unselected patients with $\mathrm{T} 1 \mathrm{DM}$ and $\mathrm{T} 2 \mathrm{DM}$ is around $20 \%$, but figures as high as $65 \%$ are reported with increasing age and diabetes duration. Because many studies were hospital based, referral bias cannot be excluded (classes II and III).

Clinical correlates or risk markers for CAN are age, diabetes duration, glycemic control, microvascular complications (DPN, retinopathy, and nephropathy), hypertension, and DLP (classes I and II).

Established risk factors for CAN are glycemic control in T1DM (class I), and a combination of hypertension, DLP, obesity, and glycemic control in T2DM (class II) [5].

\section{CLASSIFICATION OF DIABETIC AUTONOMIC NEUROPATHIES [39]}

Cardiovascular

- reduced HRV;

- resting tachycardia;

- orthostatic hypotension;

- sudden death (malignant arrhythmia).

Gastrointestinal

- diabetic gastroparesis (gastropathy);

- diabetic enteropathy (diarrhea);

- colonic hypomotility (constipation).

Urogenital

- diabetic cystopathy (neurogenic bladder);

- erectile dysfunction;

- female sexual dysfunction.

Sudomotor dysfunction

- distal hypohydrosis/anhidrosis;

- gustatory sweating.

Hypoglycemia unawareness 
Abnormal pupillary function.

\subsection{Classification of diabetic cardiovascular autonomic neuropathy [5]}

Subclinical phase

- decreased heart rates variability.

Early phase

- resting tachycardia.

Advanced stage

- excercise intolerance;

- cardiomyopathy with left ventricular dysfunction;

- orthostatic hypotension;

- silent myocardial ischaemia.

\section{SCREENING AND DIAGNOSIS}

Among the various forms of diabetic autonomic neuropathies, particularly CAN, is by far the most studied [40, 41]. Although screening for rarer atypical forms of diabetic neuropathy may be warranted, and autonomic neuropathies are the most common forms encountered in practice.

Recommendations. Suitable candidates for CAN screening are asymptomatic T2DM patients at diagnosis and T1DM patients after 5 years of disease, in particular those at greater risk for CAN due to a history of poor glycemic control $\left(\mathrm{HbA}_{1 \mathrm{c}}\right.$ $>7 \%$ ), or the presence of one major CVD risk factor (among hypertension, DLP, and smoking), or the presence of macro- or microangiopathic complications (level B).

CAN screening may be also required in asymptomatic patients for pre-operative risk assessment before major surgical procedures (level C) [5].

Symptoms and signs of autonomic neuropathy should be assessed in patients with microvascular and neuropathic complications (level E).

In the presence of symptoms or signs of CAN, tests excluding other comorbidities or drug effects/interactions that could mimic CAN should be performed (level E).

Consider assessing symptoms and signs of CAN in patients with hypoglycemia unawareness (level C) [2, 39].

CAN assessment can be used for cardiovascular risk stratification in patients with and without established CVD; as a marker for patients requiring more intensive monitoring during the perioperative period and other physiological stresses; and as an indicator for more intensive pharmacotherapeutic and lifestyle management of comorbid conditions. 


\section{CLINICAL IMPACT OF DIABETIC CARDIOVASCULAR AUTONOMIC NEUROPATHY}

\subsection{Clinical manifestations of cardiovascular autonomic neuropathy}

Symptomatic manifestations of CAN include sinus tachycardia, exercise intolerance, and $\mathrm{OH}$. Orthostatic hypotension was present in $6-32 \%$ of diabetic patients depending on diagnostic cut-offs for fall in systolic BP $(20$ or $30 \mathrm{mmHg})$ and the diabetic populations studied [5, 21, 42]. Symptoms of orthostatic intolerance were present in $4-18 \%$ of diabetic patients $[5,22]$. Orthostatic symptoms, such as light-headedness, dizziness, blurred vision, fainting, or pain in the neck or shoulder when standing, may be worse in the early morning, after meals, during a rise in core temperature, during prolonged standing, or with physical activity $[5,43,44]$. Symptoms may be disabling, are often a barrier to an effective antihypertensive treatment, and may lead to falls in the elderly.

Table 2 Abnormalities associated with cardiovascular autonomic neuropathy at the level of cardiovascular system and peripheral vascular function $[5,45,46]$

\begin{tabular}{ll}
\hline Cardiovascular system & Peripheral vascular function \\
Perioperative unstability & $\uparrow$ Peripheral blood flow and warm skin \\
Resting tachycardia & $\uparrow$ Arteriovenous shunting and swollen veins \\
Loss of reflex heart rate variations & $\uparrow$ Venous pressure \\
Hypertension & Leg and foot oedema \\
Exercise intolerance & $\begin{array}{l}\text { Loss of protective cutaneous vasomotor } \\
\text { reflexes }\end{array}$ \\
Orthostatic hypotension & $\begin{array}{l}\text { Loss of venoarteriolar reflex with } \\
\text { microvascular damage }\end{array}$ \\
Postprandial hypotension & $\uparrow$ Transcapillary leakage of macromolecules \\
Silent myocardial ischaemia & $\uparrow$ Medial arterial calcification \\
Left ventricular dysfunction and & - \\
hypertrophy & - \\
QT interval prolongation & - \\
Impaired baroreflex sensitivity & - \\
Non-dipping, reverse dipping & - \\
Sympathovagal imbalance & - \\
Dysregulation of cerebral circulation & - \\
$\downarrow$ Sympathetically mediated vasodilation of & - \\
$\uparrow$ coronary vessels & - \\
\hline Arterial stiffness &
\end{tabular}

A number of other cardiovascular abnormalities were found in association with CAN [8]. These may play a role in excess mortality and morbidity and contribute to the burden associated with CAN (Table 2). 
The most common symptoms of CAN occur upon standing and include lightheadedness, weakness, palpitations, faintness, and syncope $[5,45,46]$ (Table 2). The patient should be asked about these symptoms when a medical history is taken in the office, although the correlation of symptoms with overall autonomic deficits is weak [30, 39]. However, these symptoms may occur quite late in the disease course. It may be appropriate to screen patients with hypoglycemia unawareness, as this may be associated with CAN. [30, 39, 45, 47-50].

In its early stages, CAN may be completely asymptomatic and detected only by decreased HRV with deep breathing [5, 43, 44]. Testing HRV may be done in the office by either 1) taking an electrocardiogram (ECG) recording after standing 2) taking an ECG recording during 1-2 min of deep breathing with calculation of HRV [5, 43, 46]. In more advanced cases, patients may present with resting tachycardia $(>100 \mathrm{bpm})$ and exercise intolerance.

Table 3 Symptoms and signs associated with diabetic cardiovascular autonomic neuropathy [39]

\section{CAN}

\begin{tabular}{ll}
\hline Resting tachycardia & \\
Abnormal blood pressure regulation & Nondipping \\
& Reverse dipping \\
Orthostatic hypotension (all with standing) & Light-headedness \\
& Weakness \\
& Faintness \\
& Visual impairment \\
& Syncope \\
& Light-headedness \\
Orthostatic tachycardia or bradycardia & Weakness \\
and chronotropic incompetence (all with & Faintness \\
standing) & Dizziness \\
& Visual impairment \\
& Syncope
\end{tabular}

Exercise intolerance

Advanced disease may also be associated with $\mathrm{OH}$ (a fall in systolic or diastolic BP by $>20 \mathrm{mmHg}$ or $>10 \mathrm{mmHg}$, respectively, upon standing without an appropriate increase in HR). $\mathrm{OH}$ is usually easy to document in the office. In most cases of CAN, there is no compensatory increase in the HR, despite hypotension $[5,39,46,51]$. The diagnosis includes documentation of symptoms (Table 3) and signs of CAN, which include impaired HRV, higher resting HR, and presence of $\mathrm{OH}$. In a symptomatic patient presenting with resting tachycardia, with a history of poor glucose control, or when the diagnosis of CAN is likely, clinicians may not need to perform additional tests given costs and burden [5].

Exclusion of other comorbidities or drug effects/interactions that may present with the symptoms or signs of CAN and that mimic CAN may be needed [39, $49,52]$. In addition, polypharmacy may also directly or indirectly impact CAN [39]. 
Recommendations. Symptoms and signs of autonomic neuropathy should be assessed in patients with microvascular and neuropathic complications (level E).

In the presence of symptoms or signs of CAN, tests excluding other comorbidities or drug effects/interactions that could mimic CAN should be performed (level E) [39].

\section{CARDIOVASCULAR AUTONOMIC NEUROPATHY ASSESSMENT}

Methods of CAN assessment in clinical practice include assessment of symptoms and signs, CARTs based on HR, BP, and ambulatory blood pressure monitoring (ABPM).

\subsection{Assessment of symptoms}

Questionnaires have been developed to investigate orthostatic symptoms and their severity in dysautonomic conditions, although they have not been specifically validated for CAN and validated translations in different languages are lacking. In the Rochester Diabetic Neuropathy Study the correlation between the autonomic symptoms and the autonomic deficits was weak in T1DM and absent in T2DM patients [5, 43, 44]. Orthostatic symptoms were poorly related to fall in systolic BP on standing. For their clinical impact, orthostatic symptoms should be looked for regularly together with other dysautonomic symptoms in diabetic patients [5].

\subsection{Assessment of signs}

\subsubsection{Resting tachycardia}

Whereas abnormalities in HRV are early findings of CAN, resting tachycardia and a fixed HR are characteristic late findings in diabetic patients with vagal impairment. Resting HR of 90-100 bpm and occasional HR increments up to 130 bpm occur. The highest resting HR have been found in patients with parasympathetic damage, occurring earlier in the course of CAN than sympathetic nerve dysfunction; in those with evidence for combined vagal and sympathetic involvement, the rate returns toward normal, but remains elevated. A fixed HR that is unresponsive to moderate exercise, stress or sleep indicates almost complete cardiac denervation. A blunted HR response to adenosine receptor agonists was described in both patients with DM and patients with metabolic syndrome (MetS), and attributed to earlier stages of CAN $[8,32,53]$. Higher resting HR (> $78 \mathrm{bpm})$ compared with lower resting HR $(<58 \mathrm{bpm})$ and a rise in HR with time have been shown to be powerful, independent risk predictors for all-cause and CVD mortality in several prospective cohorts [32]. The prognostic value of resting HR is a useful tool for cardiovascular risk stratification and as a therapeutic target in high-risk patients $[5,32,36]$. Thus, HR may not provide a reliable diagnostic criterion of CAN in the absence of other causes unless it is increased by more than $100 \mathrm{bpm}$ [8].

\subsubsection{Exercise intolerance}

Autonomic dysfunction impairs exercise tolerance, reduces response in HR and BP, and blunts increases in cardiac output in response to exercise. Diabetic patients who are likely to have CAN should be tested for cardiac stress before 
undertaking an exercise program. Patients with CAN need to rely on their perceived exertion, not HR, to avoid hazardous levels of intensity of exercise. Presently, there is inadequate evidence to recommend routine screening of asymptomatic diabetic patients with an exercise ECG test. Emerging data support the utility of stress imaging testing in identifying diabetic patients with preclinical CAD, particularly patients with high-risk features, and comorbidities such as long-standing disease, CAN, multiple chronic renal failures, resting ECG abnormalities, and peripheral artery disease. The severity of CAN correlated inversely with maximal HR increase during exercise, suggesting CAN contribution to diminished exercise tolerance $[5,8,32]$.

\subsubsection{Orthostatic hypotension}

$\mathrm{OH}$ is defined as a fall in $\mathrm{BP}$ (ie, $>20 \mathrm{mmHg}$ systolic or $>10 \mathrm{mmHg}$ diastolic BP) within $3 \mathrm{~min}$ of standing and a fall in systolic BP of $30 \mathrm{mmHg}$ in response to a postural change from supine to standing. Symptoms include weakness, faintness, dizziness, visual impairment, and even syncope after a change from a lying to a standing posture. OH may become disabling, but the BP fall may also be asymptomatic. Orthostatic symptoms can be misjudged as hypoglycemia and can be aggravated by a number of drugs, including vasodilators, diuretics, phenothiazines, and particularly tricyclic antidepressants and insulin. A change from lying to standing normally results in activation of a baroreceptor-initiated, centrally mediated sympathetic reflex, resulting in an increase in peripheral vascular resistance and cardiac acceleration. In patients with $\mathrm{DM}, \mathrm{OH}$ is usually attributable to damage to the efferent sympathetic vasomotor fibers, particularly in the splanchnic vasculature. In addition, a decrease in total vascular resistance contributes to pathogenesis of this disorder [5, 43, 51].

In individuals with $\mathrm{OH}$, there may be a reduced norepinephrine response relative to the fall in BP. Reduced cardiac acceleration and cardiac output may also be important, as well as low blood volume or reduced red cell mass. Other factors such as postprandial blood pooling, the hypotensive role of insulin, and treatment of kidney or heart failure with diuretics, leading to volume depletion, could aggravate orthostatic symptoms $[5,8,32]$.

\subsubsection{Orthostatic tachycardia and bradycardia syndromes}

Symptoms compatible with orthostasis, such as feeling faint or dizzy, circumoral paresthesia, and headache, may occur on changes from a supine to an erect position and may be caused by postural tachycardia syndrome (POTS), inappropriate sinus tachycardia, neurocardiogenic syncope, or abnormalities in baroreceptor function. The hallmark of these abnormalities is the absence of a fall in BP with standing, but a tachycardia or bradycardia with the change in posture. The pathogenesis of POTS is obscure. Some patients have defective peripheral vasoconstriction and an increase in calf blood flow, whereas others have increased peripheral arterial resistance and decreased blood flow. POTS is associated with a selective defect in intraepidermal nerve fiber in the skin. Norepinephrine concentrations have been significantly related to the estimate of the severity of autonomic neuropathy, and loss of peripheral sympathetic $\mathrm{C}$ fiber tone seems to translate to inadequate cardiac venous return with thoracic hypovolemia. POTS patients have paradoxically 
unchanged plasma renin activity and low aldosterone, given their marked reduction in plasma volume. These patients also have a significant reduction in plasma erythropoietin, suggesting that the kidney may play a role in the pathogenesis of this condition. POTS patients have exaggerated muscle sympathetic nerve activity (MSNA) with baroreceptor-reflex challenges. A cadre of POTS patients have shown normal peripheral resistance and blood volume in the supine position but thoracic hypovolemia and splanchnic pooling in the upright position. Selective and maintained orthostatic pooling in the splanchnic bed occurs in low-flow POTS despite marked peripheral vasoconstriction in these patients. Local splanchnic vasoregulatory factors may counteract the vasoconstriction in these patients.In addition to these syndromes, there are selected patients with orthostatic symptoms who have a paradoxical bradycardia on standing; the symptoms closely mimic those of hypotension. It is important to recognize these differences because each is amenable to simple intervention $[5,8,32]$.

\subsubsection{QTi prolongation}

QTi prolongation has been defined as a QTc (corrected QT for HR) $\geq 460$ $\mathrm{ms}$ in women and $\geq 450 \mathrm{~ms}$ in men, although in most studies less strict criteria were used. The pathogenesis of QTi prolongation is multifactorial and includes imbalance in cardiac sympathetic innervation, intrinsic metabolic and electrolytic myocardial changes, left ventricular (LV) hypertrophy, CAD, and genetic factors could lead to QTi prolongation [38]. The day-night modulation of the QT/relative risk relation-on 24-h ECG recordings-was altered in CAN patients free of CAD, LV dysfunction, or hypertrophy, with a reversed day-night pattern and an increased nocturnal QT rate dependence [54]. Reversible QTi prolongation may be induced by hyperinsulinemia in healthy subjects, by hyperglycemia and by acute hypoglycemia in both healthy and diabetic subjects $[38,55,56]$. In T1DM patients, prolonged QTc was shown to occur frequently during overnight hypoglycemia and was associated with cardiac rate/rhythm disturbances. These findings support an arrhythmic basis for the "dead in bed" syndrome and possibly a provocative role of hypoglycemia-induced sympathetic activation in cardiovascular events [5, 57]. In a meta-analysis of 17 studies including 4584 diabetic patients, QTc prolongation ( $>441 \mathrm{~ms}$ ) was a specific (86\%) albeit insensitive (28\%) index of CAN [5].

\subsubsection{Impaired heart rate variability}

The earliest clinical indicator of CAN is a decrease in HRV. Variability in the instantaneous beat-to-beat $\mathrm{R}-\mathrm{R}$ intervals is a function of sympathetic and parasympathetic activity that regulates the cardiac functional response to the body's level of metabolic activity. In normal individuals the HR has a high degree of beat-to-beat variability and HRV fluctuates increasing with inspiration and decreasing with expiration. Initially, clinical relevance of HRV was identified through observations that fetal distress is preceded by alterations in beat-to-beat intervals before any appreciable change occurs in HR itself. The serious implications of abnormal HRV became apparent only in the late 1980s, when it was confirmed that HRV was a strong, independent predictor of mortality after acute MI [8, 46]. 


\subsubsection{Non-dipping and reverse dipping}

At night, health subjects exhibit a predominance of vagal tone and decreased sympathetic tone, associated with reduction in nocturnal BP. In diabetic CAN this pattern is altered, resulting in sympathetic predominance during sleep and subsequent nocturnal hypertension. This is associated with a higher frequency of LV hypertrophy and both fatal and severe nonfatal cardiovascular events in diabetic CAN subjects. [5, 46].

ABPM is a standard tool in hypertension research and management with regard to diagnostic, prognostic, and therapeutic issues [58]. It allows the assessment of the diurnal BP pattern, which is mainly regulated by sleep-awake changes in the autonomic cardiovascular function. ABPM may be used for research purposes to $[58]$ :

- evaluate the circadian BP pattern and its abnormalities (e.g. non-dipping, nocturnal hypertension, extreme dipping, morning surge);

- study its relationship with autonomic dysfunction, sleep disturbances, and kidney function;

- assess the 24-h BP response to treatment;

- evaluate the longer term prognostic implications of circadian BP abnormalities.

Non-dipping and reverse dipping patterns were associated with CAN, which was the major determinant of the circadian variation in BP. Several observations in both diabetic and non-diabetic patients linked non-dipping to a disruption of the circadian variation in sympathovagal activity, i.e. a diminished increase in vagal activity and a sympathetic predominance during the night. The day-night difference in systolic BP was a moderately accurate diagnostic tool for CAN, and reverse dipping as a specific (95\%)-albeit insensitive (25\%)-marker of CAN [5]. In clinical practice, ABPM in the general population is useful for diagnostic purposes and provides unique and additional information for risk stratification with regard to hypertension-related organ damage and cardiovascular events, and for the extent of BP response to treatment $[5,58]$. The European Society of Hypertension acknowledges that ABPM may improve predictions of cardiovascular risk in hypertensive patients and recommends that 24-h ABPM should be considered in the presence of either noticeable variability of office BP values or a marked discrepancy between office and home BP values, and in case of resistance to drug treatment or suspected hypotensive episodes $[5,58]$. Thus, in patients with CAN, ABPM may be particularly useful in detecting non-dipping or reverse dipping conditions, daytime postural BP changes, and postprandial hypotension, and in achieving BP control for the whole 24-h period. Conversely, in clinical practice, the presence of reverse dipping in ABPM may suggest the presence of CAN and thus requires CAN testing [5].

\subsection{8. "Silent" myocardial ischemia/cardiac denervation syndrome}

The presence of both symptomatic and asymptomatic CAD is increased in diabetic patients, and subclinical neuropathy is an important cause of SMI in patients with diabetes. Five of the 12 studies showed a statistically significant increased frequency of SMI in those with CAN compared with those without CAN [5]. "Silent" ischemia in diabetic patients can either result from CAN, from autonomic dysfunction attributable to $\mathrm{CAD}$ itself, or from both. The mechanisms 
of painless myocardial ischemia are, however, complex and not fully understood. Altered pain thresholds, subthreshold by ischemia not sufficient to induce pain and dysfunction of the afferent cardiac autonomic nerve fibers have all been suggested as possible mechanisms [32, 59]. Features of a MI in patients with CAN are silence, cough, nausea and vomiting, dyspnoe, tiredness, and ECG changes. Reduced appreciation for ischemic pain can impair timely recognition of myocardial ischemia or MI, thereby delaying appropriate therapy. Thus, patients with CAN warrant more careful attention and cardiovascular autonomic function testing might be an important component in the risk assessment of diabetic patients with CAD [32].

Features of an MI in patients with CAN are [8]:

- silence;

- cough;

- nausea and vomiting;

- dyspnea;

- tiredness;

- ECG changes.

\subsection{Cardiovascular autonomic neuropathy and sudden death}

Sudden, unexpected deaths occur among subjects with CAN. One potential cause may be severe but asymptomatic ischemia, which can induce lethal arrhythmias. QT prolongation may also predispose individuals to life-threatening cardiac arrhythmias and sudden death. Results from the European Diabetes InsulinDependent Diabetes Mellitus (IDDM) Complications Study showed that male patients with impaired HRV had a higher corrected QT prolongation than males without this complication [60]. Imaging of myocardial sympathetic innervation with various radiotracers (e.g., metaiodobenzylguanidine) has shown that predisposition to arrhythmias and an association with mortality may also be related to intracardiac sympathetic imbalance [61].

The significance of CAN as an independent cause of sudden death has, however, been questioned recently. In the Rochester Diabetic Neuropathy Study [61-63], the investigators found that all cases of sudden death in individuals with and without DM had severe CAD or LV dysfunction. They suggested that although CAN could be a contributing factor, it was not a significant independent cause of sudden death. Heart failure is, however, common in individuals with DM; it is identified in these patients by the presence of neuropathy, even in those without evidence of CAD or LV dysfunction [8]. The association of CAN in the absence of CAD and cardiomyopathy requires further study.

\subsection{Intraoperative cardiovascular lability}

Perioperative cardiovascular morbidity and mortality are increased two- to threefold in patients with diabetes. Compared with non-diabetic subjects, diabetic patients undergoing general anesthesia might experience a greater degree of decline in HR and BP during induction of anesthesia, and less of an increase after tracheal intubation and extubation. Vasopressor support is required more often in diabetic individuals with CAN than in those without CAN [32, 64]. The normal autonomic response of vasoconstriction and tachycardia does not completely compensate for the vasodilating effects of anesthesia. There is an association between CAN and 
more severe intraoperative hypothermia that can result in decreased drug metabolism and impaired wound healing. Reduced hypoxic-induced ventilatory drive [32] requires preoperative CAN screening for loss of HRV. Preoperative cardiovascular autonomic screening might provide useful information for anesthesiologists planning the anesthetic management of diabetic patients, and identify those at greater risk of intraoperative complications [8,32].

Conclusions. Thus, resting HR is not a specific sign of CAN (class IV).

After exclusion of other causes $\mathrm{OH}$ suggests an advanced CAN that should be confirmed by CARTs (class I). Orthostatic hypotension (class III), QTi prolongation (class II), and reverse dipping on ABPM are specific but insensitive indices of CAN (class III) [32].

Recommendations. In terms of recommendations, it may be advised that the presence of symptoms and/or signs is not a sufficient criterion for CAN diagnosis but should provide the motivation to perform CAN testing to get a definite diagnosis (level B). B).

Screening of orthostatic symptoms is advisable in any diabetic patient (level

Regardless of the presence of orthostatic symptoms, the $\mathrm{OH}$ test is recommended yearly, in particular in patients over the age of 50 and in hypertensive diabetic patients (level B).

CAN testing offers a useful tool to identify patients with potentially poor exercise performance and to prevent adverse outcomes when patients are introduced to exercise training programs (level C) [5, 64].

Diabetic patients with unexplained tachycardia should undergo CAN testing (level C). (level C).

Resting HR may be used in clinical practice for cardiovascular risk stratification

QTi prolongation alone is an insufficient measure of CAN but should prompt further testing (level B).

QTc may be used for cardiovascular risk stratification (level B) [5, 64].

ABPM should not be routinely employed for the diagnosis of CAN (level C).

However, it is a reliable research tool to explore 24-h BP patterns in different conditions (level B). C).

In the presence of reverse dipping, referral for CAN testing is advisable (level

ABPM may be useful in patients with CAN to detect non-dipping, to determine risk stratification for cardiovascular mortality and nephropathy progression, and to adjust antihypertensive treatment (level C) [32]. 


\section{DIAGNOSTIC TESTING FOR THE CARDIOVASCULAR AUTONOMIC NEUROPATHY}

\subsection{Cardiovascular autonomic reflex tests}

Autonomic balance involves complex interactions with several physiological mechanisms that act to maintain HR and BP within normal limits. Recent investigations have suggested that autonomic dysfunction (e.g. heightened activity of the sympathetic nervous system and suppressed activity of the parasympathetic nervous system) impairs the ability of the ANS to regulate the cardiovascular system. Thus, autonomic imbalance might be a key component involved in both the etiology and the clinical course of CVD. What is also emerging is that one needs to distinguish the difference between autonomic imbalance and clear evidence of autonomic neuropathy. Autonomic imbalance produces a number of interesting and trying clinical situations, such as orthostatic tachycardia, orthostatic bradycardia and $\mathrm{OH}$, and can be responsible for predisposition to arrhythmias and "sudden" death $[32,36]$.

CARTs assess cardiovascular autonomic function through time-domain HR response to deep breathing, Valsalva maneuver and postural change, and by measuring the end-organ response; that is, HR and BP changes. Although indirect autonomic measures, they are considered the "gold" standard in autonomic testing. Heart rate variations during deep breathing, Valsalva maneuver and lying-to-standing (HR tests) are indices mainly of parasympathetic function; whereas the OHand sustained isometric muscular strain provide indices of sympathetic function. These tests are non-invasive, safe, clinically relevant (they correlate with tests of peripheral nervous system function), easy to carry out, sensitive, specific, reproducible and standardized, and therefore they are considered consolidated, gold-standard measures of autonomic function [32].

Diagnostic tests of CAN are summarized in Table 4.

The normal, borderline and abnormal values in tests of cardiovascular autonomic functionare summarized in Table 5.

According to CAN subcommittee in the Toronto Diabetic Neuropathy Consensus Panel, CAN diagnostic criteria are divided as follows: a positive one test is early diagnosis of CAN; the presence of two or three positive tests is required for definitive diagnosis of $\mathrm{CAN}$; the presence of $\mathrm{OH}$ combined with one of the previous criteria is defined as severe CAN [5].

Table 4. Cardiovascular autonomic reflex tests [29, 42]

\begin{tabular}{cll}
\hline \multicolumn{1}{c}{ Test } & \multicolumn{1}{c}{ Technique } & Normal response and values \\
\hline Beat-to-beat HRV & $\begin{array}{ll}\text { With the patient at rest } \\
\text { and supine, heart rate is }\end{array}$ & $\begin{array}{l}\text { A difference in HR of }>15 \\
\text { beats per minute is normal }\end{array}$ \\
monitored by ECG while the & and $<10$ beats per minute is \\
patient breathes in and out at & abnormal. The lowest normal \\
6 breaths per minute, paced & value for the expiration-to \\
by a metronome or similar & inspiration ratio of the R-R \\
device & interval decreases with age: \\
& age 20-24 yr, 1.17;25-29, \\
& $1.15 ; 30-34,1.13 ; 35-39$,
\end{tabular}


Heart rate response to standing

Heart rate response to the Valsalva maneuver

Systolic blood pressure response to standing

Diastolic blood pressure response to isometric exercise
During continuous ECG monitoring, the R-R interval is measured at beats 15 and 30 after standing

The subject forcibly exhales into the mouthpiece of a manometer to $40 \mathrm{mmHg}$ for $15 \mathrm{~s}$ during ECG monitoring

$1.12 ; 40-44,1.10 ; 45-49$,

$1.08 ; 50-54,1.07 ; 55-59$, 1.06 ; 60-64, 1.04; 65-69, 1.03; and 70-75, 1.02

Normally, a tachycardia is followed by reflex bradycardia. The 30:15 ratio should be $>1.03$, borderline 1.01-1.03

Healthy subjects develop tachycardia and peripheral vasoconstriction during strain and an overshoot bradycardia and rise in BP with release. The normal ratio of longest $\mathrm{R}-\mathrm{R}$ to shortest $\mathrm{R}-\mathrm{R}$ is $>1.2$, borderline 1.11-1.2

Systolic BP is measured in the supine subject. The patient stands and the systolic BP is measured after $2 \min$

The subject squeezes a handgrip dynamometer to establish a maximum. Grip is then squeezed at $30 \%$ maximum for $5 \mathrm{~min}$
Normal response is a fall of $<10 \mathrm{mmHg}$, borderline fall is a fall of $10-29 \mathrm{mmHg}$ and abnormal fall is a decrease of $>30 \mathrm{mmHg}$

The normal response for diastolic BP is a rise of $>$ $16 \mathrm{mmHg}$ in the other arm, borderline $11-15 \mathrm{mmHg}$

Table 5 Normal, borderline and abnormal values in tests of cardiovascular autonomic function [27]

\begin{tabular}{|c|c|c|c|}
\hline & Normal & Borderline & Abnormal \\
\hline \multicolumn{4}{|l|}{$\begin{array}{l}\text { Tests reflecting mainly } \\
\text { parasympathetic } \\
\text { function }\end{array}$} \\
\hline $\begin{array}{l}\text { Heart rate response to } \\
\text { Valsalva Manoeuvre } \\
\text { (Valsalva ratio) }\end{array}$ & $\geq 1.21$ & $1.11-1.20$ & $\leq 1.10$ \\
\hline $\begin{array}{l}\text { Heart rate (R-R } \\
\text { interval) variation }\end{array}$ & $\geq 15$ beats $/ \mathrm{min}$ & $11-14$ beats/min & $\leq 10$ beats $/ \mathrm{min}$ \\
\hline $\begin{array}{l}\text { During deep breathing } \\
\text { (maximum-minimum } \\
\text { heart rate) immediate } \\
\text { heart rate response to } \\
\text { standing ( } 30: 15 \text { ratio) }\end{array}$ & $\geq 1.04$ & $1.01-1.03$ & $\leq 1.00$ \\
\hline
\end{tabular}


Tests reflecting mainly

sympathetic function

Blood pressure
response to standing
(fall in systolic blood
$\mathrm{mmHg} \mathrm{mmHg} \mathrm{mmHg}$
pressure)

Blood pressure
response to sustained

$\leq 10$

$11-29$

$\geq 30$

handgrip (increase

in diastolic blood

pressure

The Toronto Diabetic Neuropathy Consensus Panel [5] have concluded the following regarding diagnosis of CAN:

- the following CARTs are the "gold" standard for clinical autonomic testing: HR response to deep breathing, standing and Valsalva maneuver, and BP response to standing (class II evidence);

- these CARTs are sensitive, specific, reproducible, easy to carry out, safe and standardized (classes II and III);

- the Valsalva maneuver is not advisable in the presence of proliferative retinopathy and when there is an increased risk of retinal hemorrhage (class IV); III);

- CARTs are subject to a number of confounding or interfering factors (class

- age is the most relevant factor affecting HR tests (class I);

- a definite diagnosis of CAN and CAN staging requires more than one HR test and the $\mathrm{OH}$ test (class III).

The main clinical indications of the autonomic reflex tests $[5,52,65]$ :

- diagnosis and staging of CAN in T2DM patients (at diagnosis and annually thereafter);

- diagnosis and staging of CAN in T1DM patients (5 years after diagnosis and annually thereafter);

- dtratification of cardiovascular risk: in pre-operatory testing, pre-physical activity, indication of selective beta-blocker, and suspected silent ischemia;

- differential diagnosis of other manifestations of CAN (regardless of DM duration): assess whether gastroparesis, erectile dysfunction, $\mathrm{OH}$, dizziness, syncope, or tachycardia in diabetic persons are due to dysautonomia;

- evaluate the progression of autonomic failure and monitor response to therapy (e.g., continuous infusion of insulin, post-transplants, and use of antioxidants);

- differential diagnosis of other causes of neuropathy such as autoimmune autonomic neuropathy (chronic inflammatory demyelinating polyneuropathy, celiac disease, amyotrophy) or toxic-infectious neuropathy (alcohol, primary neuritic Hansen's disease, human immunodeficiency virus) as well as in cases where the presence of autonomic neuropathy is disproportionate to the sensory-motor neuropathy. 
The most sensitive and specific diagnostic tests currently available to evaluate CAN in clinical research are: (1) HRV; (2) baroreflex sensitivity (BRS); (3) MSNA; (4) plasma catecholamines, and (5) heart sympathetic imaging [50, 66].

\subsection{Short-term ECG}

The short-term ECG recordings can be analyzed by dedicated software in the frequency domain. This method usually uses the Fourier method, which transform R-R intervals into waves with three basis components: very low frequency $\leq 0.04$ $\mathrm{Hz}$ (VLF); low frequency $0.04-0.15 \mathrm{~Hz}$ (LF) and high frequency 0.15-0.4 Hz (HF). HF represents vagal activity, whereas LF combines the effect of sympathetic and parasympathetic influence. A decrease in HF is a sign of parasympathetic dysfunction, in the early stages of autonomic dysfunction in DM, when sympathetic predominance is observed it leads to an increase in $\mathrm{LF} / \mathrm{HF}$ [67, 68]. It is not clear if classical Ewing's tests or time-domain methods are better for diagnosis of CAN. However, Ewing's tests are simpler and can be more easily implemented during routine clinical use.

\subsection{Heart rate variability}

Heart rate is never completely stable. Continuous tonic, phasic, and transient external and internal stimuli of multiple origins affect HR to a variable but measurable extent. Five different mechanisms have been described:

(1) sympathetic and parasympathetic efferences to the sinus node;

(2) neurohumoral influences (e.g. catecholamines, thyroid hormones);

(3) stretch of the sinus node;

(4) changes in local temperature;

(5) ionic changes in the sinus node.

Under resting conditions, it can be assumed that the short-term HRV is essentially determined by the first and third factors. The sympathetic and parasympathetic stimuli directly influence HR and are responsible for a physiologic variation in the HR, or HRV. The HRV can be evaluated in the time and frequency domains $[5,50,66]$.

Time domain measures of the normal R-R intervals include the difference between the longest and shortest R-R intervals, the standard deviation of 5-min average of normal R-R intervals (SDANN), and the root-mean square of the difference of successive R-R intervals (RMSSD). Longer recordings (e.g. 24-h, classic $24 \mathrm{~h}$ Holter ECG) allow the calculation of additional indices, as the number of instances per hour in which two consecutive R-R intervals differ by more than $50 \mathrm{~ms}$ over $24 \mathrm{~h}$ (pNN50). Essentially, all these indices explore the parasympathetic activity [67].

In the frequency domain, the use of spectral analysis of R-R interval (and other cardiovascular and respiratory signals) allows a precise description of the different fluctuations. The components of the HRV obtained by spectral analysis provide information about both the sympathetic and parasympathetic influences on the heart [5]. Based on studies using acceptable techniques, there is evidence of reduced parasympathetic modulation of HR in diabetes and also reduced modulation of systolic BP in the low-frequency region $[5,68,69]$ particularly after sympathetic 
stimulation in response to tilting, or in the microcirculation. As most of the CARTs essentially explore the parasympathetic activity, there is no other simple test of sympathetic activity capable of identifying early (functional or anatomic) autonomic sympathetic abnormality [66]. CARTs are considered the "gold" standard for CAN testing. Impaired HRV time- and frequency-domain indices have been reported in diabetic patients before CARTs abnormalities arise. However, the few studies that assessed the diagnostic accuracy against the reference standard of CARTs found only fair results. Time- and frequency-domain analysis of 24-h ECG recordings has documented an abnormal nocturnal sympathetic predominance in diabetic patients that was linked to BP non-dipping. In obese patients weight loss was associated with an improvement in global HRV and in parasympathetic HRV indices $[35,66]$.

In this way, HRV testing is a clinically relevant measure in addition to CARTs and provides key information about autonomic-parasympathetic and sympatheticmodulation of the cardiovascular system. Analysis of HRV can be done using statistical indices in the time and frequency domains. Time-domain indices of global HRV and total spectral power of HRV represent the index of parasympathetic activity, as well as the HRV spectral power in the HF region, while the relative proportion (not the absolute power) in the LF of HRV provides a relative measure of sympathetic modulation. This interpretation should be made with cautions if respiratory artifacts (slow breaths) cannot be excluded. Application of the technique is critically dependent upon understanding of the underlying physiology, the mathematical analyses used, and the many confounders and possible technical artefacts [50, 67-69].

In this way, misinterpretation of power spectrum takes place due to irregular respiratory pattern and verbalization during breathing, creating artefactual LF and false "sympathetic overactivity". Lack of spectral decomposition algorithm when using autoregressive methodology. Use of the absolute power of R-R interval LF spectral data as evidence of sympathetic activity. In case of very low HRV (2-4\% of total variability found in healthy subjects) the interpretation of spectral components is affected by the presence of non-autonomic components in the respiratory range. Other confounding factors (such as drugs) similar as those reported for CARTs [50]. It is obvious that reduction in HRV is associated with CAN, but this method has no standard values for diagnosis CAN [70]. Also during $24 \mathrm{~h}$ recording many factors can have an influence on HRV parameters, such as concomitant illness, use of medication, and lifestyle factors (exercise, stress, smoking etc.).

Recommendations. The best approach to HRV testing involves the analysis of ECG recordings in conjunction with respiration and beat-to-beat BP recordings (level C).

When respiration cannot be recorded, breathing rate should be controlled (15 breaths/min), and hyperventilation or slow deep breathing avoided (level B).

The subjects must not speak during recordings (level $\mathbf{C}$ ).

The optimal recording time is $4-5$ min during well controlled rest. Longer times $(7 \mathrm{~min})$ may be preferable if fast Fourier transform methods are used and if frequent ectopics are to be edited. Long uncontrolled recording times should be avoided (level C). 
When testing is done under stable conditions, autoregressive or fast Fourier transform methods can be used. When fast changes are to be expected (e.g. during interventions) autoregressive algorithms are preferred, or alternatively special timevarying techniques; age-related reference curve should be obtained for the healthy population in the same environment and using the methodology adopted, construct 95\% confidence limits (level B); other recommendations on confounding factors are similar as those reported for CARTs; used with the appropriate methodology HRV has an increasingly important role in clinical research and therapeutic trials [50].

During 24-h recordings: if the goal is to define the circadian pattern of autonomic activity, long-duration spectra (e.g. $1 \mathrm{~h}$ ) and autoregressive algorithms are preferable; if the goal is to define relatively faster modifications, shorter time windows (e.g. $5 \mathrm{~min}$ ) are preferable. Special time-varying techniques can provide beat-to-beat autonomic changes [50].

\subsection{Heart rate turbulence}

Another Holter-based technique for evaluating CAN is the HR turbulence (HRT). HRT refers to sinus rhythm cycle length fluctuations following isolated premature ventricular beats. After an initial acceleration, the sinus rate decelerates after a premature ventricular beat. There are 2 components of HRT; turbulence onset and turbulence slope. A transient vagal inhibition triggers the mentioned initial acceleration in HR as a response to the missed baroreflex afferent input due to hemodynamically ineffective ventricular contraction. The successive deceleration in HR is caused by a sympathetically mediated overshoot of arterial pressure through vagal recruitment. HRT evaluation can be used in the risk assessment after acute $\mathrm{MI}$ and in the monitoring of disease progression in heart failure and CAN [71,72]. A turbulence slope of below $3.32 \mathrm{msec} / \mathrm{R}-\mathrm{R}$ is $97 \%$ sensitive and $71 \%$ specific for the diagnosis of CAN as detected by the CARTs in patients with T2DM [72].

\subsection{Baroreflex sensitivity}

The BRS is an interesting approach as it combines information derived from both HR and BP. The measurement of the cardiac-vagal arm BRS can be done with several methods: drugs or physical manoeuvres can be applied to modify BP; alternatively, spontaneous BP variations can be used. In all cases the response in HR to the changes in BP is quantified. None of the BRS tests available todaybased on drug-induced or physically induced changes in BP, spontaneous BP fluctuations with the sequences technique or spectral analysis-have shown so far a definite advantage over the others, or a clinically relevant difference [73].

Longitudinal studies have demonstrated that BRS has important independent prognostic value in cardiac patients $[50,66,73]$ and in diabetic patients. Although some observations in diabetic patients support an early impairment of BRS before CARTs abnormalities, very few studies have evaluated so far the diagnostic accuracy of BRS measures as compared with the reference standard of CARTs with inconsistent results. Thus, no definite conclusion is possible on the diagnostic characteristics for CAN of BRS assessment, in particular on its sensitivity. In patients without CAN an early stage of functional BRS abnormalities [50, 74] still 
responsive to life-style intervention - physical training or dietary improvement and weight reduction has been documented. BRS assessment may warrant use for identifying subjects at risk for CAN and also in clinical trials [50].

In this way, cardiac vagal BRS assessment is an important component of autonomic testing as it combines information derived from both HR and BP. Cardiac vagal BRS is a widely recognised independent prognostic index for cardiovascular mortality and morbidity in the general-mainly cardiac and the diabetic population (class II).

No definite conclusion is possible on the diagnostic characteristics of BRS assessment (classes III-IV).

The presence of early abnormalities with respect to CARTs and their reversibility with appropriate treatments warrant the clinical use of BRS in identifying subjects at risk for CAN and to test potential therapeutic approaches (classes II-III).

Pharmacological methods allow assessment of BRS across a range of physiologically relevant $\mathrm{BP}$ and when used with microneurography-measurement of the sympathetic baroreflex. But this invasive technique is limited to research purposes. The methodology of BRS (in particular spontaneous BRS) is simple and fast. All BRS techniques require a dedicated beat-to-beat non-invasive BP monitor. None of the BRS tests today available have shown a definite advantage over the others, nor a clinically relevant difference (class II) [50]. Fluctuations induced by drifts of the non-invasive BP monitors. Most methods need a large number of arbitrary constraints imposed by the calculations that may affect the results. Respiratory pattern: although BRS measures in general do not need a strict control of respiratory pattern, slow breathing increases BRS and reduces sympathetic efferent drive; therefore, some feedback from respiration is necessary to correctly interpret the results. Age-related reduction in BRS. Other confounding factors (e.g. drugs) are similar as those for CARTs [50].

If the spontaneous approach is adopted, it is suggested to use a battery of methods based on the simplest single 5 min recording procedure (spontaneous BRS) and present the results in terms of a central measure (average or median) (level C) [50]: recording should be performed during spontaneous breathing for 4-5 min, under monitored respiration, or during controlled breathing at 15 breaths/ min (level C); pre-filtering of the data improves the agreement between methods and provides a more robust estimate of BRS (level C); the recording time should be kept between 4 and $5 \mathrm{~min}$ of well-controlled rest. Avoid long uncontrolled recording timesthe subjects must not speak during recordings (level C); age-related reference curves should be obtained from the healthy population of in the same environment and for the methodology adopted, and construct $95 \%$ confidence limits (level B); other recommendations on confounding factors are similar as those reported for CARTs [50].

\subsection{Muscle sympathetic nerve activity}

Increased resting MSNA and blunted responsiveness to physiological hyperinsulinaemia or glucose ingestion have been described in T2DM having neuroadrenergic autonomic dysfunction, and resembles insulin-resistant states and obesity. MSNA abnormalities in these conditions reverse with weight loss [50,66]. 
In contrast, T1DM is associated with a significant decrease in the number of bursts, by about half [75]. Although reproducibility is similar to non-diabetic subjects, obtaining good quality recordings is much more difficult in patients with DPN than in non-diabetic subjects $[50,66]$, presumably as a result of a reduction in the conducting sympathetic nerve fibres.In this way, the MSNA is the only method allowing direct and continuous measurement of sympathetic nerve traffic (class I). MSNA is the only method that can directly assess the sympathetic vascular arm of the arterial or cardiopulmonary baroreflex (class I). Type 1 diabetes appears to be associated with a reduction of MSNA (class IV). In early T2DM, resting MSNA might be increased, possibly due to hyperinsulinemia (class IV). The technique is difficult, invasive, time-consuming, requires specialized trained operator and cannot be repeated often in the same subject (class II) [50]. Confounders. BP variation; large inter-individual variations; food intake; age;posture; hypoxia; hydration; exercise; female reproductive hormones; arousal; sleep; mental stress; ethnicity [50].

Recommendations. MSNA should not be routinely employed for the diagnosis of CAN (level C). MSNA should be employed with standard CARTs or for specific tests aimed at measuring vascular sympathetic modifications (e.g. glycemic clamps) (level C) [50].

\subsection{Catecholamine assessment and cardiovascular sympathetic tests}

Norepinephrine plasma appearance rate is in principle the biochemical equivalent of MSNA. Norepinephrine plasma appearance rate and clearance have been determined in idiopathic autonomic neuropathy as well as in diabetic CAN. While norepinephrine clearance is low in idiopathic autonomic neuropathy, this was not the case in CAN, and accordingly in diabetic CAN no additional diagnostic power was added by the inclusion of $\left[{ }^{3} \mathrm{H}\right]$-norepinephrine kinetic studies $[50,76]$. Thus, catecholamine kinetics is an interesting technique which may give more information about catecholamine production and clearance across different regions but is unsuitable to be used as a diagnostic tool yet. Plasma dihydroxyphenylalanine (DOPA) is not related to sympathetic neuropathy and has a mixed neuronal and non-neuronal origin. Plasma 3,4-dihydroxyphenylglycol (DHPG) may be a more sensitive marker of overall sympathetic innervation than supine plasma norepinephrine [50], and simultaneous measurement of norepinephrine and DHPG yields more information than measurement of either alone. Catecholamine assessment in DM showed in general lower than normal responses to postural changes, exercise, hypoglycemia, and CARTs. A subnormal orthostatic increment in plasma norepinephrine is a specific but not sensitive index of baroreflex-sympathoneural failure or sympathetic noradrenergic denervation [50].

Clinical investigations including catecholamine determinations have contributed significantly to the understanding of the pathophysiology of CAN (class III). In the diagnostic context, the significance has been less prominent, partly due to the limited inclusion of the essays in clinical evaluations.

Plasma catecholamine concentrations can indicate sympathetic noradrenergic and adrenomedullary hormonal system activity. Because levels of catecholamines are extremely responsive to lifestyle factors such as posture, temperature, dietary intake, medications, distress, and comorbidities, the clinical diagnostic value of 
plasma levels of catecholamines depends importantly on controlling or monitoring these factors (class III). Whole-body plasma norepinephrine and epinephrine respond rather slowly (min) to different physiological manoeuvres. During turnover studies, different regional norepinephrine and epinephrine activities are "diluted" into a large plasma pool, contributing to blunted responses. Standardization of experimental conditions is to a large extent prohibitive for clinical routine purposes. In general, there is no neurochemical index that specifically assesses cardiac sympathetic innervation or function. This requires measurement of norepinephrine entry entry of into the venous drainage of the heart, in turn requiring right heart catheterization, measurement of coronary sinus blood flow, and infusion of tracerlabelled norepinephrine [50].

Confounders. Plasma norepinephrine concentrations increase with age. Thus, age matching is mandatory for comparisons. Smoking increases sympathetic nervous activity and catecholamine concentrations-24 h tobacco abstention is required for comparisons. Posture, emotional stress, and ambient temperature all affect catecholamine concentrations and should thus be standardized [50].

Recommendations. In a number of experimental conditions, plasma catecholamine measurements are mandatory. For clinical routine diagnosis and staging of CAN the usefulness of plasma catecholamine concentrations is less obvious (level C). Plasma norepinephrine, epinephrine, and DHPG concentrations should be measured when whole-body sympathetic activity is assessed together with other relevant physiological parameters (HR, BP, cardiac output, hormonal and metabolic events) [50].

\subsection{Heart sympathetic imaging and heart function tests}

Direct assessment of cardiac sympathetic innervation is possible using radiolabelled catecholamines or sympathomimetic amines that are actively taken up by sympathetic nerve terminals. Although in principle, it is possible to directly assess the integrity of both the parasympathetic as well as the sympathetic nervous system, there has been a paucity of research on parasympathetic imaging of the heart. Cardiac sympathetic neuroimaging, before and after administration of particular pharmacologic probes, can assess specific aspects of neuronal function. This combination has rarely been used [50].

Four tracers have been utilized to visualize the sympathetic nervous innervation of the heart: $\left[{ }^{123} \mathrm{I}\right]$-meta-iodobenzylguanidine (MIBG), $\left[{ }^{11} \mathrm{C}\right]$-meta-hydroxyephedrine (HED), 6- $\left[{ }^{18} \mathrm{~F}\right]$ dopamine, and $\left[{ }^{11} \mathrm{C}\right]$-epinephrine [50, 77-79].

The washout rates from the myocardium of $\left[{ }^{11} \mathrm{C}\right]$-epinephrine or $6-\left[{ }^{18} \mathrm{~F}\right]$ dopamine can give information on vesicular integrity. In subjects with T1DM and $\mathrm{CAN}$, the washout rates of $\left[{ }^{11} \mathrm{C}\right]$-epinephrine parallels those of $\left[{ }^{11} \mathrm{C}\right]-\mathrm{HED}$, suggesting regional differences in vesicular uptake or retention. Causes of defective tracer uptake or increased washout from the heart are a matter of current research [50, $80]$.

The interpretation of findings using sympathetic neurotransmitter analogues is complicated by the fact that alterations in sympathetic nervous system tone may also affect the retention of these tracers, and this fact is often not considered as an explanation for the clinical findings. In the isolated rat heart model, elevated norepinephrine concentrations in the perfusion increased neuronal HED clearance 
rates consistent with the concept that neuronal "recycling" of HED can be disrupted by increased synaptic norepinephrine levels. Alternatively at high norepinephrine concentrations, non-neuronal uptake of HED into myocardial cells and impaired retention may be an interfering factor [50, 80].

Additionally, interpretation of early myocardial [ $\left.{ }^{123} \mathrm{I}\right]-\mathrm{MIBG}$ retention is complicated by increased BMI and diastolic BP which have been reported to reduce myocardial MIBG uptake. Moreover, difficulties and delays in acquisition of utilizable images can complicate the interpretation of the measurement obtained. The delivery of tracers is critically influenced by myocardial perfusion, so myocardial retention of tracers should be performed with a quantitative analysis of myocardial blood flow. This can be performed using positron emission tomography in order to derive a myocardial retention index [50,81]. However, although regional perfusion deficiencies can be excluded using single photon emission computed tomography, quantitative analysis of regional myocardial perfusion cannot be performed. Additionally, myocardial ischaemia or damage is also known to result in cardiac denervation which may occur in the absence of alterations in CARTs [33, 79], whereas CAN is associated with impaired vasodilatory capacity in response to adenosine. Anoxic ischaemia severely decreases the efficiency of vesicular sequestration and thus accelerates the loss of radioactivity, giving the false impression of denervation. LV dysfunction in DM has also been reported to reduce [23I]-MIBG retention and increased washout rate [50].

Highlights. Scintigraphic tracers directly assess the structural integrity of the sympathetic nervous system supply to the heart (class III). [ $\left.{ }^{123} \mathrm{I}\right]-\mathrm{MIBG}$ scanning and single photon emission computed tomography are widely used and available at most secondary care institutions; however, MIBG scanning is approved and reimbursed for evaluation of pheochromocytoma and so far not for evaluation of cardiac sympathetic innervation. Most data relate to the evaluation of cardiac sympathetic integrity; few studies evaluate the respiratory system. The relationships of deficits in tracer uptake/wash-out to sympathetic neuronal integrity and function are poorly understood: current tracers may not be the most optimum.

Combined neuroimaging-pharmacologic approaches are required. Scintigraphic data correlates with HRV testing, but have greater sensitivity to detect changes in sympathetic neuronal structure and/or function [50, 82] (class III).

Scintigraphic data correlate with indices of myocardial perfusion and LV dysfunction in T1DM (class III). Limited studies demonstrate that decreased "uptake" and excessive "washout" of MIBG-derived radioactivity is an adverse prognostic finding in a spectrum of conditions including DM and that scintigraphic data are affected by the quality of glucose control (class III). Cost of scintigraphic studies is considerable [50, 78, 79].

Confounders. Parasympathetic tracers are not yet generally available. $\left[{ }^{11} \mathrm{C}\right]-$ HED and $6-\left[{ }^{18} \mathrm{~F}\right]$-dopamine positron emission tomography have limited availability and are not reimbursed. Damage to the myocardium and LV dysfunction interferes with tracer uptake and washout independently of changes in CARTs. Regional myocardial [ $\left.{ }^{123} \mathrm{I}\right]-\mathrm{MIBG}$ "uptake" is semi-quantitative and not a clean index of neuronal uptake, which occurs extremely rapidly. [ $\left.{ }^{123} \mathrm{I}\right]-\mathrm{MIBG}$ retention is affected by BMI, diastolic BP, and local factors which influence the tracer uptake and retention. Delivery of tracers is critically influenced by myocardial perfusion 
(myocardial retention of tracers should be performed with quantitative analysis of myocardial blood flow) [50].

The effects of the following on the kinetics of myocardial tracer retention are poorly understood: age (except for $6-\left[{ }^{18} \mathrm{~F}\right]$-dopamine), gender, glucose, insulin, DLP, hypertension, vasoactive agents. Methodology for the assessment of sympathetic integrity is not standardized. Normative values have not been developed [50].

Recommendations. Scintigraphic studies should not be routinely employed for the diagnosis of CAN and should be utilized in concert with standard CARTs (level C); scintigraphic studies are extremely valuable in the identification of sympathetic noradrenergic denervation as a mechanism of neurogenic $\mathrm{OH}$ (level B); [ $\left.{ }^{123} \mathrm{I}\right]-\mathrm{MIBG}$ single photon emission computed tomography offers semiquantitative assessment and $\left[{ }^{11} \mathrm{C}\right]-\mathrm{HED}, 6-\left[{ }^{18} \mathrm{~F}\right]$-dopamine, and $\left[{ }^{11} \mathrm{C}\right]$-epinephrine positron emission tomography offer quantitative assessment of cardiac sympathetic integrity (level B); there is no standardized methodology for scintigraphic assessment of cardiac sympathetic integrity and only limited data on the reproducibility exist (level C); scintigraphic tracer uptake is affected by myocardial perfusion, and tracer retention is affected by available energy for the active neuronal and vesicular uptake transporters (level C); the results of scintigraphy should be compared with an appropriate control population (level C); scintigraphic studies offer good sensitivity to detect sympathetic neuronal loss in the heart (level C); scintigraphy is appropriate to explore the effects of sympathetic denervation on cardiac physiology, metabolism, and function (level C); scintigraphy is useful as a marker of cardiac sympathetic denervation in cross-sectional and longitudinal research studies (level C) [50].

\subsection{Diagnostic Testing for Orthostatic Symptoms}

A standard test for establishing the cause of postural symptoms is the headup tilt-table study. Although this is technician and physician intensive, it often resolves the cause of the symptoms. Typically, autonomic labs will combine tilttable and other studies with HRV measures obtained during deep breathing and Valsalva. Other functional syndromes may also be revealed, such as the vasoconstrictor syndrome (paradoxic orthostatic hypertensive syndrome, also known as $\mathrm{OH}$ ) and paradoxic orthostatic bradycardia syndrome [8].

\subsection{Diagnostic algorithm for diabetic cardiovascular autonomic neuropathy (Table 6)}

\subsection{Differential diagnosis of diabetic neuropathies}

Consider excluding neuropathy with causes other than diabetes (Table 7) by undertaking a family and medication history and performing relevant investigations (e.g., serum $\mathrm{B}_{12}$, folic acid, thyroid function, complete blood count, metabolic panel, and a serum protein immunoelectrophoresis) [49].

\subsection{Neuropathy end points for research and clinical practice [3, 39]}

The recommended CAN measures for clinical trials targeting either a specific intervention or for prognostic implications include:standardized CARTs that are 
simple, sensitive, specific, reproducible, and assess the changes in the R-R interval on ECG recordings in response to simple clinical maneuvers (deep breathing, Valsalva, and standing) $[5,39,49,83,84]$; indices of $\operatorname{HRV}[39,45,50,85]$; resting HR and QTc $[28,34,39,86]$; other methods such as baroreflex sensitivity, cardiac sympathetic imaging, and microneurography require sophisticated infrastructure and highly trained personnel and are quite expensive and timeconsuming $[5,39,50,87]$.

7.13. Diagnostic criteria for cardiovascular autonomic neuropathy

No unanimous criteria for diagnosis of CAN have been adopted to date. $[3,39]$

Table 6 Diagnostic algorithm for diabetic cardiovascular autonomic neuropathy

\begin{tabular}{|c|c|c|c|}
\hline & Symptoms & Signs/diagnostic tests & Differential workup \\
\hline \multirow[t]{9}{*}{$\begin{array}{l}\text { Resting } \\
\text { tachycardia }\end{array}$} & $\begin{array}{l}\text { Palpitations } \\
\text { Could be } \\
\text { asymptomatic }\end{array}$ & $\begin{array}{l}\text { Clinical exam: resting } \\
\text { heart rate }>100 \mathrm{bpm}\end{array}$ & Anemia \\
\hline & & & Hyperthyroidism \\
\hline & & & Fever \\
\hline & & & $\begin{array}{l}\text { CVD (atrial } \\
\text { fibrillation, flutter, } \\
\text { other) }\end{array}$ \\
\hline & & & Dehydration \\
\hline & & & Adrenal insufficiency \\
\hline & & & Some medications \\
\hline & & & $\begin{array}{l}\text { Smoking, alcohol, } \\
\text { caffeine }\end{array}$ \\
\hline & & & $\begin{array}{l}\text { Recreational } \\
\text { drugs (cocaine, } \\
\text { amphetamines, } \\
\text { methamphetamine, } \\
\text { mephedrone) }\end{array}$ \\
\hline \multirow[t]{5}{*}{$\begin{array}{l}\text { Orthostatic } \\
\text { hypotension }\end{array}$} & Light-headedness & $\begin{array}{l}\text { Clinical exam: a } \\
\text { reduction of }>20 \mathrm{mmHg} \\
\text { in the systolic blood } \\
\text { pressure or }>10 \mathrm{mmHg} \text { in } \\
\text { diastolic blood pressure }\end{array}$ & Adrenal insufficiency \\
\hline & Weakness & & $\begin{array}{l}\text { Intravascular volume } \\
\text { depletion }\end{array}$ \\
\hline & Faintness & & $\begin{array}{l}\text { Blood loss/acute } \\
\text { anemia }\end{array}$ \\
\hline & $\begin{array}{l}\text { Visual } \\
\text { impairment }\end{array}$ & & Dehydration \\
\hline & Syncope & & $\begin{array}{l}\text { Pregnancy/ } \\
\text { postpartum }\end{array}$ \\
\hline
\end{tabular}


CVD

Alcohol

Medication

Antiadrenergics

Antianginals

Antiarrhythmics

Anticholinergics

Diuretics

ACE inhibitors/

angiotensin receptor

blocker

Narcotics

Neuroleptics

Sedatives

Table 7 Differential diagnosis of diabetic neuropathies [39]

Metabolic disease

Systemic disease

Infectious

Inflammatory

Nutritional

Drugs

Metals
Thyroid disease (common)

Renal disease

Systemic vasculitis

Nonsystemic vasculitis

Paraproteinemia (common)

Amyloidosis

HIV

Hepatitis B

Lyme

Chronic inflammatory demyelinating polyradiculoneuropathy

B12

Postgastroplasty

Pyridoxine

Thiamine

Tocopherol

Industrial agents, drugs, and metals

Industrial agents

Acrylamide

Organophosphorous agents

Alcohol

Amiodarone

Colchicine

Dapsone

Vinka alkaloids

Platinum

Taxol

Arsenic

Mercury 
A single abnormal result among the two or three HR tests actually performed was considered a sufficient criterion for early CAN diagnosis. However, the presence of abnormalities in more than one test on several occasions was indicated as preferable for diagnosis $[5,29]$. In addition, the presence of two or three abnormal results (two for borderline, three for definite) among the seven autonomic cardiovascular indices (including the five standard CARTs and other time and frequency domain indices of $\mathrm{HRV}$ ) was recommended as a criterion for CAN diagnosis [88].

The CAN Subcommittee of the Toronto Consensus Panel on Diabetic Neuropathy established four reasons why the diagnosis of CAN is relevant to clinical practice [5]:

- for diagnosing and staging the different clinical forms of CAN: initial, definite, and advanced or severe;

- for the differential diagnosis of clinical manifestations (e.g. resting tachycardia, $\mathrm{OH}$, and dyspnea upon exercise) and their respective treatment;

- for stratifying the degree of cardiovascular risk and the risk of other diabetic complications (nephropathy, retinopathy, and SMI);

- to adapt the goal of $\mathrm{HbA}_{1 \mathrm{c}}$ in each patient: for example, those with severe CAN should have a less aggressive glycemic control due to the risk of asymptomatic hypoglycemia in these patients while patients with initial stages of CAN should have a more intensive glycemic control.

CARTs are the "gold" standard clinical tests for cardiovascular autonomic neuropathy [5]. Following the $8^{\text {th }}$ International Symposium on Diabetic Neuropathy in 2010, criteria for diagnosis and staging of CAN are defined in the CAN Subcommittee of the Toronto Consensus Panel statement $[5,6]$. Accordingly, only one abnormal CART result is sufficient to diagnose possible or early CAN; among the 7 autonomic function analysis (5 CARTs, time-domain and frequency-domain HRV tests), 2 or 3 abnormal tests indicate definite or confirmed CAN; and severe/ advanced CAN can be indicated by concurrent $\mathrm{OH}[5,72]$.

\subsection{Staging of cardiovascular autonomic neuropathy}

Ewing et al. proposed a classification based on "early involvement" (one abnormal result on HR test or two borderline results), "definite involvement" (two or more abnormal results on HR tests), and "severe involvement" (presence of $\mathrm{OH}$ [42]. An "autonomic neuropathy score" - obtained by scoring the results of CARTs has been used with the dual advantage of quantifying the progression of $\mathrm{CAN}$ and providing an overall quantitative result [5]. While an abnormal $\mathrm{OH}$ test result generally occurs late in DM and subsequent to abnormalities in the HR tests, no chronological order or a markedly different prevalence of abnormalities among the HR tests has been found [5, 88]. Considering progression from an early to an advanced involvement, instead of from parasympathetic to sympathetic neuropathy, would appear to be the most appropriate approach to CAN staging, although $\mathrm{OH}$ may on rare occasions precede abnormalities in HR tests [42]. The available information regarding the duration required to progress from an earlier 
to a later stage of CARTs impairment is scant and it is not documented that a progression to $\mathrm{OH}$ and symptomatic forms invariably occurs in all patients. The combination of CARTs with tests for sudomotor function may provide a more accurate diagnosis of diabetic autonomic neuropathy [5].

Conclusions. The following CARTs are the "gold" standard for clinical autonomic testing: HR response to deep breathing, standing, and Valsalva manoeuvre, and BP response to standing (class II); these CARTs are sensitive, specific, reproducible, easy to perform, safe and standardized (classes II and III); the Valsalva manoeuvre is not advisable in the presence of proliferative retinopathy and when there is an increased risk of retinal haemorrhage (class IV); CARTs are subject to a number of confounding or interfering factors (class III). Age is the most relevant factor affecting HR tests (class I); a definite diagnosis of CAN and CAN staging requires more than one HR test and the $\mathrm{OH}$ test (class III) [5].

Recommendations. Diagnosis of CAN is based on the use of CARTs for HR response to deep breathing, standing, Valsalva manoeuvre, and for BP response to standing (level A); for the diagnosis and monitoring of CAN, more than one HR test and the $\mathrm{OH}$ test are required (level B); performance of CARTs should be standardized and the influence of confounding variables minimized (level A); agerelated normal ranges of HR tests are strictly required (level A) [5].

CAN diagnosis and staging: (1) the presence of one abnormal cardiovagal test result identifies the condition of possible or early CAN, to be confirmed over time; (2) at least two abnormal cardiovagal results are required for a definite or confirmed diagnosis of $\mathrm{CAN}$; and (3) the presence of $\mathrm{OH}$ in addition to HR test abnormalities identifies severe or advanced CAN (level B); CARTs allow CAN staging from early to advanced involvement (level C); progressive stages of CAN are associated with increasingly worse prognosis (level B) [5].

\section{PREVENTION OF THE DIABETIC CARDIOVASCULAR AUTONOMIC NEUROPATHY}

Prevention of diabetic neuropathies focuses on glucose control and lifestyle modifications. Available evidence pertains only to CAN, and most of the large trials that have evaluated the effect of glucose control on the risk of complications have included CAN as secondary outcomes or as post hoc analyses rather than as primary outcomes. In addition, in some of these trials, the outcome measures used to evaluate neuropathy may have limited ability to detect a benefit, if present [39].

Recommendations. Optimize glucose control as early as possible to prevent or delay the development of CAN in people with T1DM (class A); consider a multifactorial approach targeting glycaemia among other risk factors to prevent CAN in people with T2DM (class C) [39]. 


\section{TREATMENT OF THE DIABETIC CARDIOVASCULAR AUTONOMIC NEUROPATHY}

Recommendations. Optimize glucose control as early as possible to prevent or delay the development of CAN in people with T1DM (class A); consider amultifactorial approach targeting glycemia among other risk factors to prevent CAN in people with T2DM (class C); consider lifestyle modifications to improve CAN in patients with prediabetes (class C). As with diabetic simmetrial sensory neuropathy (DSPN), multiple other therapies targeting various pathogenetic mechanisms have failed to reverse established CAN. CAN treatment is generally focused on alleviating symptoms and should be targeted to the specific clinical manifestation [39].

Pathogenetic treatment of CAN includes: balanced diet and physical activity; optimization of glycemic control; treatment of DLP; antioxidants, first of all $\alpha$-lipoic acid (ALA), aldose reductase inhibitors, acetyl-L-carnitine; vitamins, first of all fat-soluble vitamin $\mathrm{B}_{1}$; correction of vascular endothelial dysfunction; prevention and treatment of thrombosis; in severe cases-treatment of $\mathrm{OH}$ [89].

\subsection{Intensive glucose control}

Intensive glucose control designed to achieve near-normal glycemia reduced the risk of incident CAN during the DCCT by $45 \%$ and by $31 \%$ in its follow-up study, the Epidemiology of Diabetes Interventions and Complications (EDIC) study $[39,48]$. The highly reproducible and sensitive testing protocol, the robust definitions used for CAN, and the large sample size in DCCT/EDIC enhance the validity of the results and support the rationale for implementing and maintaining tight glucose control as early as possible in the course of T1DM. In contrast, glycemic control in T2DM has not consistently lowered the risk of CAN [39, 47]. However, a multifactorial intervention, including a lifestyle component, targeting glucose and cardiovascular disease risk factors reduced the risk of CAN by $60 \%$ in people with T2DM [24, 39].

\subsection{Lifestyle modifications}

Nutrition and physical activity. Correction of obesity. Limit salt intake to 2-4 g/d. Limit smoking, alcohol, foods that contain caffeine. It has been established that compliance with recommended lifestyle modifications (exercise, weight loss, etc.) help improve insulin sensitivity level. Sedentary lifestyle (less than $1000 \mathrm{kcal} / \mathrm{wk}$ ) is accompanied by the risk of mortality three times higher than when living an active lifestyle [90].

There is no consensus regarding dietary regimens, and although the Diabetes Prevention Program (DPP) used a low-calorie, low-fat diet, others have championed a Mediterranean diet that is moderately lower in carbohydrate $(45 \%)$ and higher in fat $(35 \%-40 \%)$, with less than $10 \%$ of saturated fat. The ADA does not recommend a specific diet over another for the diabetic patients. However, it clearly states that the total amount of fat should represent $25-35 \%$, and saturated fat of $<7 \%$ of the total calories, with monounsaturated fat as the major source of fat intake. It also points out the importance of adequate amounts of fruits, vegetables, and whole wheat cereals. On the other hand, the ADA lists three different types of 
diets (either low-carbohydrate, or low-fat calorie-restricted or Mediterranean diet) as a mean for weight loss for individuals who have or are at risk of having DM. Most of the studies showed favorable effects of Mediterranean diet on glycemic control and CVD, although a certain degree of controversy remains regarding some issues, such as obesity. Decreasing OS, inflammation and IR are all possible mechanism by which Mediterranean diet pauses as a protective dietary pattern. Concerning cardiovascular risk modification, there is some cardiovascular benefit of adhering to a Mediterranean diet in diabetic patients; although there are no studies looking at the cardiovascular outcome in diabetic patients only. This fact necessitates further studies to assess the effect of adherence to Mediterranean diet on cardiovascular risk in diabetic patients [90, 91]. Although the DPP [39, 92]. The Impaired Glucose Tolerance Neuropathy (IGTN) study [39, 93] reported benefits of lifestyle interventions on measures of CAN and DSPN, respectively, these trials did not include subjects with established diabetes. In addition, in the DPP, indices of CAN improved with the lifestyle intervention and did not change in the other arms $[39,92]$.

The best models to date regarding parameters for an evidence-based, intensive lifestyle intervention come from the DPP, the Steno-2 Study, the Italian supervised treadmill study [94], and the University of Utah T2DM study [95]. The latter study recently reported nerve fiber regeneration in patients with T2DM engaged in an exercise program compared with loss of nerve fibers in those who only followed standard of care. Overall, such an approach focuses on either exercise alone (supervised aerobic and/or resistance training) [94,95] or combined dietary modification and exercise [39].

Autonomic neuropathy can increase the risk of exercise-induced injury or adverse events through decreased cardiac responsiveness to exercise, $\mathrm{OH}$, impaired thermoregulation, impaired night vision due to impaired papillary reaction, and greater susceptibility to hypoglycemia [5]. CAN is also an independent risk factor for cardiovascular death and SMI [28]. Therefore, individuals with diabetic CAN should undergo cardiac investigation before beginning physical activity more intense than that to which they are accustomed [3].

Both peripheral and autonomic neuropathies are characterized by a progressive loss of nerve fiber function. Most peripheral neuropathy affects the extremities, particularly the lower legs and the feet, but also the hands, whereas damage to the ANS may lead to imbalances between the sympathetic and parasympathetic nerve fibers that innervate the heart and blood vessels, as well as abnormalities in HR control and vascular dynamics. To prescribe or engage in exercise that is both safe and effective, health care providers and patients with DM need to increase their understanding of the pathophysiological nature of neuropathies and the physical activity hurdles that may arise from the presence of a neuropathy. With proper care and preventative measures, patients with DM that experience either type of neuropathy can benefit from regular participation in mild to moderate aerobic, resistance, and balance activities, assuming they take any potential alterations into account to ensure that exercise is safe and effective [96, 97]. Dosed physical activity reduces hyperinsulinemia and encourages the tendency to normalize lipid metabolism in addition to body weight decrease. Physical activity is associated with higher HRV and lower HR, therefore may be a predictor of positive changes 
in HRV indices [14]. Obtaining the necessary amount of energy combined with physiologic food ration forms the dietary principles. Individuals with CAN should be screened and receive physician approval and possibly an exercise stress test before exercise initiation. Exercise intensity is best prescribed using the HRreserve method with direct measurement of maximal HR [96, 97].

Recommendations. Individuals with autonomic neuropathy (particularly CAN) should avoid high-intensity physical activities unless they have been cleared by a physician to participate: they should also avoid physical exertion in hot or cold environments since dehydration may be a risk for those who have difficulty with thermoregulation; individuals must be made aware that hypotension may occur after vigorous activities; recumbent cycling or water aerobics may be safer activities for individuals with $\mathrm{OH}$; for better accuracy, individuals should monitor exercise intensity using the HR reserve method using a measured maximal HR, if possible, or use perceived exertion. The results indicate that 6-mo aerobic exercise training improves the cardiac ANS function in T2DM patients. However, more favourable effects are found in T2DM patients with definite CAN. [98].

\subsection{Glucose control}

The DCCTand the follow-up observational EDIC study (DCCT/EDIC), which recently marked its 30th year, stands as the pivotal trial demonstrating clear and persistent benefits of hight glucose control for both DSPN and CAN in patients with T1DM [47, 48, 95, 99-101]. DCCT enrolled 1,441 patients with T1DM who were randomly assigned to intensive or conventional insulin therapy $[47,48,102-$ 104]. Both DSPN and CAN were uncommon at DCCT start, in part owing to the intentional exclusion of persons with symptomatic neuropathy [47, 48, 104]. After an average of 6.5 years of follow-up, $\mathrm{HbA}_{1 \mathrm{c}}$ was $7.4 \%$ in the intensive group and $9.1 \%$ in the conventional group [47, 102]. Adjusting for the presence of confirmed DSPN at baseline, the risk reduction for incident DSPN with intensive glucose control during DCCT was $64 \%[47,48,102,103]$. The prevalence of CAN almost doubled in the conventional group by DCCT-end, while remaining static in the intensive group $[47,102,105]$. The risk reduction in incident CAN with intensive therapy during DCCT was 45\% [47, 48, 102, 104, 105]. The DCCT/EDIC has furthered the understanding of the role of glucose control in the development and progression of neuropathy [47, 48, 104].

To summarize findings from the DCCT/EDIC study, the differences in the incidence and prevalence of both DSPN and CAN reflect differences in glucose control, favoring $\mathrm{HbA}_{1 \mathrm{c}}$ levels that are closer to nondiabetic levels. These findings parallel the reported long-term benefits of prior intensive glycemic control on retinopathy, nephropathy [25, 47], and CVD [47, 106], a phenomenon termed "metabolic memory" [100].

The Kumamoto trial was the first randomized controlled trial to report beneficial effects of tight glucose control $\left(\mathrm{HbA}_{1 \mathrm{c}}\right.$ of $7.4 \%$ at trial end), as compared with conventional glucose control $\left(\mathrm{HbA}_{1 \mathrm{c}} 9.4 \%\right.$ at trial end), in preventing PNP deterioration in 110 Japanese participants with T2DM followed for a mean of 6 years; no differences were observed on CAN measures [47, 107].

The United Kingdom Prospective Diabetes Study (UKPDS) trial enrolled 3.867 relatively young patients with newly diagnosed T2DM. By the end of the trial 
(10-year average), intensive glucose control $\left(\mathrm{HbA}_{1 \mathrm{c}}<7 \%\right)$ had no effect on DSPN or CAN, as compared with standard control $\left(\mathrm{HbA}_{1 \mathrm{c}}<7.9 \%\right)$, although some marginal benefit was observed in those followed for 15 years $[47,108]$. The use of insensitive DSPN or CAN measures, the rather narrow difference in the $\mathrm{HbA}_{1 \mathrm{c}}$ between arms, and potential differences in other risk factors for T2DM and DSPN could have contributed to these findings. Since no measures of neuropathy were assessed during the observational follow-up of the UKPDS, unveiling a potential long-term beneficial "legacy effect", as observed for cardiovascular outcomes [47, 109], was not possible [47, 108].

The Veteran Affairs Diabetes Trial (VADT) randomized 1,791 veterans with T2DM (baseline $\mathrm{HbA}_{1 \mathrm{c}} 9.4 \%$ ) to either intensive or standard glucose control. After approximately 5.6 years of follow-up, there were no differences in the rates of new DSPN in the intensive versus standard arm (38\% vs. $40 \%$, respectively), despite significant differences in the mean HbA1c between groups (6.9\% vs. $8.4 \%$, respectively). The study reported a nonsignificant increase in CAN in the intensive group and $8.2 \%$ versus $5.2 \%$ [47, 110]. The effects of glucose control on DSPN/ CAN development in this elderly cohort with multiple comorbidities are difficult to interpret, especially given that reliance on self-report to define neuropathy outcomes has a large potential for bias [111].

The Anglo-Danish-Dutch Study of Intensive Treatment in People with Screen Detected Diabetes in Primary Care (ADDITION) study enrolled more than 1,500 individuals with newly diagnosed T2DM. No difference in the prevalence of DSPN or CAN between the intensively treated and standard groups was observed after 6-year follow-up [112, 113]. Although the participants enrolled in the ADDITION trial were very early in their disease course and, theoretically, more susceptible to glucose control intervention, important limitations are to be considered. ADDITON did not obtain baseline evaluations for DSPN or CAN, preventing objective evaluations of change in DSPN or CAN with intervention [47, 113, 114]. Per trial design, ADDITION randomized the health-care providers and their respective practices, and not the participants, to intensive or standard treatment. At the end of the trial, it was evident that most providers intensified treatment as much as possible; as a consequence, the $\mathrm{HbA}_{1 \mathrm{c}}$ was identical in both groups $(6.4 \%)$ [47, $113,114]$.

The ACCORD trial enrolled $\sim 10,000$ patients with T2DM at high risk for $\mathrm{CVD}$ events and examined the effects of intensive glucose control (target $\mathrm{HbA}_{1 \mathrm{c}}$ $<6 \%)$ versus standard of care $\left(\mathrm{HbA}_{1 \mathrm{c}} \sim 7 \%\right)$ on $\mathrm{CVD}$ and other complications. The main DSPN measure was the Michigan Neuropathy Screening Instrument (MNSI), with individual components of the MNSI included in the prespecified analytic outcomes. Although the intensive glycemic intervention arm of ACCORD was stopped early due to an increase in all-cause mortality [47, 111] and glycemic control was relaxed in this arm, follow-up continued for the entire 5 years as originally planned. A treatment group difference in the $\mathrm{HbAlc}$ of $1 \%$ was maintained at 3.8 years, and at study end, the incidence of DSPN was significantly reduced in the intensive, as compared with the standard, treatment group. Incidence of loss of ankle reflexes was also lower in the intensive, as compared with the standard, arm; however, the loss of vibration perception threshold (the DSPN measure evaluated in UKPDS) was not significantly different [112]. Ongoing 
analyses from ACCORD (currently in submission) further evaluate the effects of glycemic control and the rest of the interventions in this cohort on DSPN and CAN [47].

The Steno-2 trial showed that intensive multifactorial intervention targeting glucose, BP, lipids, smoking, and other lifestyle factors in a European cohort of patients with T2DM had no effect on DSPN, although it reduced the progression/ development of CAN [22, 47].

DCCT/EDIC and other smaller trials strongly demonstrated that intensive control designed to achieve near-normal glycemia is essential to preventing or delaying progression of DSPN and CAN in T1DM. The highly reproducible and sensitive testing protocol repeated over time in DCCT/EDIC, the robust definitions used for DSPN and CAN, and the large sample size enhance the validity of the results $[47,48,104]$. Although the prevalence of these complications is low in young people with short T1DM duration, DSPN and CAN develop over time. DCCT/EDIC showed that implementing and maintaining tight glucose control as early as possible in the course of T1DM prevents early neuropathy development and promotes long-time protection, especially for CAN [48, 104]. The emergence of DSPN and CAN during EDIC was highly but not entirely glucose dependent $[48,104,111]$, suggesting that the level of glucose control achieved during DCCT is difficult to sustain over time and, perhaps, insufficient to fully prevent adverse nervous system effects $[48,104,111]$.

The presence of multiple comorbidities and risk factors, including hypertension, DLP, and obesity, among most T2DM patients included in these trials, the polypharmacy required to reach glucose targets, and the high incidence of hypoglycemia and weight gain might have attenuated the effects of glucose control and contributed to inconsistent findings among T2DM study populations [48, 104, $111]$.

Selection of specific glucose-lowering strategies and/or combining more effectively pharmacological and lifestyle interventions may be required in future T2DM trials and in clinical practice to obtain a reduction in DSPN and CAN among T2DM patients.

\subsection{Drugs for treatment of hypercholesterolemia}

\subsubsection{The 3-hydroxy-3-methylglutaryl-coenzyme (HMG-CoA) reductase inhibitors}

HMG-CoA reductase inhibitors (statins: lovastatin, pravastatin, fluvastatin, simvastatin, atorvastatin, rosuvastatin, pitavastatin).

Statins reduce the synthesis of cholesterol in the liver by competitively inhibiting HMG-CoA reductase activity. The reduction in intracellular cholesterol concentration induces an increased expression of thelow density lipoprotein receptor (LDLR) on the surface of the hepatocytes, which results in increased uptake of LDL-C from the blood and a decreased plasma concentration of LDL-C and other apolipoprotein B (apoB)-containing lipoproteins, including TG-rich particles. Current available evidence from meta-analyses suggests that the clinical benefit is largely independent of the type of statin but depends on the extent of LDL-C lowering, 
therefore the type of statin used should reflect the LDL-C goal in a given patient [115].

Secondary prevention statin studies such as MRC/BHF Heart Protection Study (HPS) showed significant risk reduction among individuals with DM. Based on this, the primary prevention of CVD with atorvastatin in T2DM in the Collaborative Atorvastatin Diabetes Study (CARDS) was designed to assess the effects of aggressive lipid lowering on the primary prevention of atherosclerotic CVD (ASCVD) in individuals with T2DM. In individuals with average or mildly elevated LDL-C at baseline (mean $117 \mathrm{mg} / \mathrm{dL}$ ), an LDL-C reduction to a mean of $82 \mathrm{mg} /$ $\mathrm{dL}$ was accompanied by a $37 \%$ reduction in major cardiovascular events compared with placebo. CARDS, which originally planned a mean follow-up of 4 years, was terminated 2 years early because of the significant benefit achieved in the statin group [116, 117].

Information about the statins therapies, starting daily dosages and dosage ranges; high-intensity and moderate-intensity statin therapy; metabolic effects and main considerations are given in Tables 8-10.

Table 8 Statins therapies, starting daily dosages and dosage ranges [117]

\begin{tabular}{|l|c|c|}
\hline \multicolumn{1}{|c|}{ Statins } & Starting daily dosage & Dosage range \\
\hline Atorvastatin & $10-20 \mathrm{mg}$ & $10-80 \mathrm{mg}$ \\
\hline Fluvastatin & $40 \mathrm{mg}$ & $20-80 \mathrm{mg}$ \\
\hline Lovastatin & $20 \mathrm{mg}$ & $10-80 \mathrm{mg}$ \\
\hline Pitavastatin & $2 \mathrm{mg}$ & $2-4 \mathrm{mg}$ \\
\hline Pravastatin & $40 \mathrm{mg}$ & $10-80 \mathrm{mg}$ \\
\hline Rosuvastatin & $10 \mathrm{mg}$ & $5-40 \mathrm{mg}$ \\
\hline Simvastatin & $20-40 \mathrm{mg}$ & $5-80 \mathrm{mg}^{\mathrm{a}}$ \\
\hline
\end{tabular}

Table 9 High-intensity and moderate-intensity statin therapy* [117]

\begin{tabular}{|l|l|}
\hline & \\
\hline $\begin{array}{l}\text { High-intensity statin therapy (lowers LDL } \\
\text { cholesterol by } \geq 50 \% \text { ) }\end{array}$ & $\begin{array}{l}\text { Moderate-intensity statin therapy (lowers } \\
\text { LDL cholesterol by 30\% to }<50 \% \text { ) }\end{array}$ \\
\hline Atorvastatin $40-80 \mathrm{mg}$ & Atorvastatin $10-20 \mathrm{mg}$ \\
\hline Rosuvastatin $20-40 \mathrm{mg}$ & Rosuvastatin $5-10 \mathrm{mg}$ \\
\hline & Simvastatin $20-40 \mathrm{mg}$ \\
\hline & Pravastatin $40-80 \mathrm{mg}$ \\
\hline & Lovastatin $40 \mathrm{mg}$ \\
\hline & Fluvastatin XL $80 \mathrm{mg}$ \\
\hline & Pitavastatin 2-4 mg \\
\hline & \\
\hline
\end{tabular}


*Once-daily dosing. XL, extended release; LDL cholesterol: low-density lipoprotein cholesterol.

In summary, statins [117]:

$\downarrow$ LDL-C $21-55 \%$ by competitively inhibiting rate-limiting step of cholesterol synthesis in the liver, leading to upregulation of hepatic LDL receptors (primarily);

$\downarrow$ TG $6-30 \%$ and $\uparrow$ high-density lipoprotein (HDL-C) (2-10\%).

Table 10 Statins: Metabolic effect and main considerations [117]

\begin{tabular}{|c|c|c|}
\hline Drug class & Metabolic effect & Main considerations \\
\hline $\begin{array}{l}\text { HMG-CoA reductase } \\
\text { inhibitors (statins: } \\
\text { lovastatin, pravastatin, } \\
\text { fluvastatin, simvastatin, } \\
\text { atorvastatin, rosuvastatin, } \\
\text { pitavastatin) }\end{array}$ & $\begin{array}{l}\text { Primarily } \downarrow \text { LDL-C } 21- \\
55 \% \text { by competitively } \\
\text { inhibiting rate-limiting step } \\
\text { of cholesterol synthesis } \\
\text { in the liver, leading to } \\
\text { upregulation of hepatic } \\
\text { LDL receptors } \\
\text { Effects on TG and HDL-C } \\
\text { are less pronounced ( } \downarrow \\
\text { TG 6-30\% and } \uparrow \text { HDL-C } \\
2-10 \%)\end{array}$ & $\begin{array}{l}\text { Liver function test prior to } \\
\text { therapy and as clinically indicated } \\
\text { thereafter } \\
\text { Myalgies and muscle weakness in } \\
\text { some patients } \\
\text { Potential for drug-drug } \\
\text { interaction between some statins } \\
\text { and CYP450 3A4 inhibitors, } \\
\text { cyclosporine, warfarin, and } \\
\text { protease inhibitors } \\
\text { Myopathy/rhabdomyolysis in rare } \\
\text { cases; increased risk with co- } \\
\text { administration of some drugs (see } \\
\text { product labeling) } \\
\text { Simvastatin dosages of } 80 \text { mg are } \\
\text { no longer recommended } \\
\text { Do not exceed } 20 \text { mg simvastatin } \\
\text { daily with amlodipine or } \\
\text { ranolazine } \\
\text { Plasma elevations of rosuvastatin } \\
\text { may be higher among Asian } \\
\text { persons than other ethnic groups } \\
\text { New-onset diabetes is increased } \\
\text { in patients treated with statins; } \\
\text { however, it is dose-related, } \\
\text { occurs primarily in patients with } \\
\text { MetS, appears to be less common } \\
\text { with pravastatin and possibly } \\
\text { pitavastatin, and occurs overall to } \\
\text { a lesser extent than the associated } \\
\text { decrease in ASCVD }\end{array}$ \\
\hline
\end{tabular}

HMG-CoA: hydroxymethylglutaryl-coenzyme A; LDL-C: low-density lipoprotein cholesterol; TG: triglycerides; HDL-C: high-density lipoprotein cholesterol; CYP450 3A4 inhibitors: cytochrome P450 3A4 inhibitors; MetS: metabolic syndrome; ASCVD: arteriosclerosis cardiovascular disease. 


\subsubsection{Cholesterol absorbion (ezetimibe)}

Ezetimibe is the first lipid-lowering drug that inhibits intestinal uptake of dietary and biliary cholesterol without affecting the absorption of fat-soluble nutrients. By inhibiting cholesterol absorption at the level of the brush border of the intestine (by interaction with the Niemann-Pick C1-like protein 1 (NPC1L1), ezetimibe reduces the amount of cholesterol delivered to the liver. In response to reduced cholesterol delivery, the liver reacts by upregulating LDLR expression, which in turn leads to increased clearance of LDL-C from the blood [115].

Information on the cholesterol absorption inhibitors, metabolic effects and main considerations are given in Table 11.

\begin{tabular}{|c|c|c|}
\hline Drug class & Metabolic effect & Main considerations \\
\hline $\begin{array}{l}\text { Cholesterol absorption } \\
\text { inhibitors (ezetimibe) }\end{array}$ & $\begin{array}{l}\text { Primarily } \downarrow \text { LDL-C } 10 \text { - } \\
18 \% \text { by inhibiting intestinal } \\
\text { absorption of cholesterol and } \\
\text { decreasing delivery to the } \\
\text { liver, leading to upregulation } \\
\text { of hepatic LDL receptors } \\
\downarrow \text { Apo B } 11-16 \% \\
\text { In combination with statins, } \\
\text { additional } \downarrow \text { LDL-C } 25 \% \text {, } \\
\text { total } \downarrow \text { LDL-C } 34-61 \% \\
\text { In combination with } \\
\text { fenofibrate, } \downarrow \text { LDL-C } 20 \text { - } \\
22 \% \text { and } \downarrow \text { apo B } 25-26 \% \\
\text { without reducing } \uparrow \text { HDL-C }\end{array}$ & $\begin{array}{l}\text { Myopathy/rhabdomyolysis } \\
\text { (rare) } \\
\text { When co-administered } \\
\text { with statins or fenofibrate, } \\
\text { risks associated with } \\
\text { those drugs remain (e.g., } \\
\text { myopathy/rhabdomyolysis, } \\
\text { cholelithiasis) }\end{array}$ \\
\hline
\end{tabular}

LDL-C: low-density lipoprotein cholesterol; Apo: apolipoprotein; HDL-C: highdensity lipoprotein cholesterol.

In summary, cholesterol absorption inhibitors [117]:

$\downarrow$ LDL-C $10-18 \%$ by inhibiting intestinal absorption of cholesterol and decreasing delivery to the liver, leading to upregulation of hepatic LDL receptors (primarily);

$\downarrow$ ApoB 11-16\%;

$\downarrow$ LDL-C $25 \%$, total LDL-C $34-61 \%$ (in combination with statins);

$\downarrow$ LDL-C $20-22 \%$ and apo B $25-26 \%$ without reducing increasing HDL-C (in combination with fenofibrate).

Ezetimibe. Usual recommended starting daily dosage $10 \mathrm{mg}$; dosage range $10 \mathrm{mg}$ [117].

9.4.3. PCSK9 inhibitors (Proprotein convertase subtilisin/kexin type 9) inhibitors (alirocumab, evolocumab)

Recently a new class of drugs, PCSK9 inhibitors, has become available that targets a protein (PCSK9) involved in the control of the LDLR [118]. 
Table 12 PCSK9 (Proprotein convertase subtilisin/kexin type 9) inhibitors: Metabolic effect and main considerations [117]

\begin{tabular}{|c|c|c|}
\hline Drug class & Metabolic effect & Main considerations \\
\hline $\begin{array}{l}\text { PCSK9 (Proprotein } \\
\text { convertase subtilisin/ } \\
\text { kexin type 9) inhibitors } \\
\text { (alirocumab, evolocumab) }\end{array}$ & $\begin{array}{l}\downarrow \text { LDL-C } 48-71 \%, \downarrow \\
\text { non-HDL-C } 49-58 \%, \downarrow \\
\text { Total-C } 36-42 \%, \downarrow \text { Apo } \\
\text { B } 42-55 \% \text { by inhibiting } \\
\text { PCSK9 binding with } \\
\text { LDLRs, increasing } \\
\text { the number of LDLRs } \\
\text { available to clear LDL, } \\
\text { and lowering LDL-C } \\
\text { levels }\end{array}$ & $\begin{array}{l}\text { Requires subQ self-injection, and } \\
\text { refrigeration is generally needed } \\
\text { Adverse reactions resulted in } \\
\text { discontinuation in } 2.2 \% \text { overall, } \\
1.2 \% \text { more than placebo for } \\
\text { evolocumab, and } 5.3 \% \text { overall, } \\
0.2 \% \text { more than placebo for } \\
\text { alirocumab. Overall levels } \\
\text { of adverse reactions and } \\
\text { discontinuation very low } \\
\text { Adverse reactions with } \\
\text { significantly different rates } \\
\text { between drug and placebo were } \\
\text { local injection site reactions (1.9\% } \\
\text { greater for alirocumab vs. placebo, } \\
0.7 \% \text { greater for evolocumab vs. } \\
\text { placebo) and influenza (1.2\% } \\
\text { greater for alirocumab vs. placebo, } \\
0.2 \% \text { for evolocumab vs. placebo). } \\
\text { The most common adverse } \\
\text { reactions with similar rates for drug } \\
\text { vs. placebo were for the following: } \\
\text { Alirocumab (4-12\%; most common } \\
\text { to least common): nasopharyngitis, } \\
\text { influenza, urinary tract infections, } \\
\text { diarrhea, bronchitis, and myalgia } \\
\text { Evolocumb (2-4\%; most common } \\
\text { to least common): Nasopharyngitis, } \\
\text { back pain, and upper respiratory } \\
\text { tract infection }\end{array}$ \\
\hline
\end{tabular}

LDL-C: low-density lipoprotein cholesterol; HDL-C: high-density lipoprotein cholesterol; TC: total cholesterol; Apo: apolipoprotein; LDLR: low-density lipoprotein receptor; SubQ: subcutaneous.

Information about the PCSK9 (Proprotein convertase subtilisin/kexin type 9) inhibitors (alirocumab, evolocumab), metabolic effects and main considerations are given in Table 12.

Two monoclonal antibody inhibitors of PCSK9, a protein that regulates the recycling of LDLR, have recently been approved by the Food and Drug Administration (FDA) [119].

Alirocumab and evolucumab are subcutaneously injectable LDL-lowering agents capable of further reducing LDL approximately $60 \%$ when added to maximum statin therapy $[120,121]$. Their strong efficacy in reducing LDL-C and possible synergistic effects with statins, combined with a favorable safety profile and 
tolerability, give these drugs the potential to revolutionize the treatment of the highest risk individuals, as well as those individuals unable to reach LDL-C goals with maximally tolerated statin dose $[122,123]$. This drug class meets a large unmet need for more aggressive lipid-lowering therapy beyond statins in an effort to further reduce residual risk in individuals with clinical ASCVD and DM, which up to now has not been possible [117].

Elevated levels/functions of this protein in plasma reduce LDLR expression by promoting, upon binding, the LDLR lysosomal catabolism and increase plasma LDL-C concentration, while lower levels/functions of PCSK9 are related to lower plasma LDL-C levels [124]. Therapeutic strategies have been developed mainly using monoclonal antibodies that reduce LDL-C levels by $\sim 60 \%$ independent from the presence of a background lipid-lowering therapy.

The mechanism of action relates to the reduction of plasma levels of PCSK9, which in turn is not available to bind the LDLR. Since this interaction triggers the intracellular degradation of the LDLR, lower levels of circulating PCSK9 will result in a higher expression of LDLRs at the cell surface and therefore in a reduction of circulating LDL-C levels [115, 124].

In summary, PCSK9 inhibitors [117]:

$\downarrow$ LDL-C 48-71\%, $\downarrow$ non-HDL-C 49- 58\%;

$\downarrow$ Total cholesterol $36-42 \%$;

$\downarrow$ ApoB $42-55 \%$ by inhibiting PCSK9 binding with LDLRs;

$\uparrow$ the number of LDLRs available to clear LDL);

$\downarrow$ LDL-C levels.

Alirocumab. Usual recommended starting daily dosage $75 \mathrm{mg}$ every 2 weeks; dosage range $75-150 \mathrm{mg}$ every 2 weeks.

Evolocumab. Usual recommended starting daily dosage $140 \mathrm{mg}$ every 2 weeks or $420 \mathrm{mg}$ once mo; dosage range not applicable [117].

9.4.4. Fibric acid derivatives (gemfibrozil, fenofibrate, fenofibric acid)

Fibrates are agonists of peroxisome proliferator-activated receptor- $\alpha$ (PPAR- $\alpha$ ), acting via transcription factors regulating various steps in lipid and lipoprotein metabolism. By interacting with PPAR- $\alpha$, fibrates recruit different cofactors and regulate gene expression. As a consequence, fibrates have good efficacy in lowering fasting TG levels as well as postprandial TGs and TG-rich lipoprotein remnant particles. The HDL-C raising effects of fibrates are modest [115, 125].

In summary, fibrates [117]:

$\downarrow$ TG $20-35 \%$ (primarily);

$\uparrow$ HDL-C $6-18 \%$ by stimulating lipoprotein lipase activity;

$\downarrow$ TC and LDL-C 20-25\% (fenobibrate);

$\downarrow$ very-low-density lipoprotein cholesterol (VLDL-C) and LDL-C;

reciprocal rise in LDL-C transforms the profile into a less atherogenic form by shifting fewer LDL particles to larger size;

$\downarrow$ fibrinogen level (fenobibrate).

Fenofibrate. Usual recommended starting daily dosage 48-145 mg; dosage range $48-145 \mathrm{mg}$.

Gemfibrozil. Usual recommended starting daily dosage $1.200 \mathrm{mg}$; dosage range $1.200 \mathrm{mg}$. 
Fenofibric acid. Usual recommended starting daily dosage 45-135 mg; dosage range 45-135 $\mathrm{mg}$ [117].

Information about the fibric acid derivates: Metabolic effect and main considerations are given in Table 13.

Table 13 Fibric acid derivates: Metabolic effect and main considerations [117]

\begin{tabular}{|c|c|c|}
\hline Drug class & Metabolic effect & Main considerations \\
\hline $\begin{array}{l}\text { Fibric acid } \\
\text { derivatives } \\
\text { (gemfibrozil, } \\
\text { fenofibrate, } \\
\text { fenofibric acid) }\end{array}$ & \begin{tabular}{|l|} 
Primarily $\downarrow$ TG 20 - \\
$35 \%$, \\
$\uparrow$ HDL-C 6-18\% \\
by stimulating \\
lipoprotein lipase \\
activity \\
Fenofibrate may $\downarrow$ TC \\
and LDL-C $20-25 \%$ \\
Lower VLDL-C and \\
LDL-C; reciprocal \\
rise in LDL-C \\
transforms the profile \\
into a less atherogenic \\
form by shifting fewer \\
LDL particles to \\
larger size \\
Fenofibrate $\downarrow$ \\
fibrinogen level
\end{tabular} & $\begin{array}{l}\text { Gemfibrozil may } \uparrow \text { LDL-C } 10-15 \% \\
\text { GI symptoms, possible cholelithiasis } \\
\text { May potentiate effects of orally } \\
\text { administered anticoagulants } \\
\text { Gemfibrozil may } \uparrow \text { fibrinogen levela } \\
\text { Gemfibrozil and fenofibrate can } \uparrow \\
\text { homocysteine independent of vitamin } \\
\text { concentrationsb } \\
\text { Myopathy/rhabdomyolysis when used } \\
\text { with statin (uncommon with gemfibrozil, } \\
\text { but increased risk with all statins except } \\
\text { fluvastatin); interaction less likely with } \\
\text { fenofibrate or fenofibric acid (no apparent } \\
\text { difference by statin) } \\
\text { Fibrates are associated with increased } \\
\text { serum creatinine levels, which may not } \\
\text { reflect renal dysfunction } \\
\text { Fenofibrate dose should be cut by two- } \\
\text { thirds and gemofibrozil by one-half when } \\
\text { eGFR is } 15-60, \text { and fibrates should be } \\
\text { avoided when eGFR is }<15 \\
\text { May cause muscle disorders } \\
\text { Can improve diabetic retinopathy }\end{array}$ \\
\hline
\end{tabular}

TG: triglycerides; HDL-C: high-density lipoprotein cholesterol; TC: total cholesterol; VLDL-C: very low-density lipoprotein cholesterol; LDL-C: low-density lipoprotein cholesterol; eGFR: estimated glomerular filtration rate; aresults vary. Gemfibrozil has been shown to decrease, have no effect on, or increase fibrinogen depending on the study; bresults vary. Gemfibrozil has been shown to have no effect on or increase homocysteine.

\subsubsection{Niacin (nicotinic acid)}

Nicotinic acid has been reported to decrease fatty acid influx to the liver and the secretion of VLDL by the liver; this effect appears to be mediated in part by the effects on hormone-sensitive lipase in the adipose tissue. Nicotinic acid has key action sites in both liver and adipose tissue. In the liver nicotinic acid is reported to inhibit diacylglycerol acyltransferase-2 (DGAT-2) that results in the decreased secretion of VLDL particles from the liver, which is also reflected in reductions of both IDL and LDL particles. Nicotinic acid raises HDL-C and apolipoprotein A1 (apoA1) primarily by stimulating apoA1 production in the liver 
[126]. The effects of nicotinic acid on lipolysis and fatty acid mobilization in adipocytes are well established.

Information about the fibric acid derivates: Metabolic effect and main considerations are given in Table 14. [117]

Table 14 Niacin (nicotinic acid): Metabolic effect and main considerations

\begin{tabular}{|c|c|c|}
\hline Drug class & Metabolic effect & Main considerations \\
\hline Niacin (nicotinic acid) & $\begin{array}{l}\text { LDL-C } 10-25 \%, \downarrow \text { TG } 20- \\
30 \%, \\
\text { HDL-C } \uparrow 10-35 \% \text { by } \\
\text { decreasing hepatic synthesis } \\
\text { of LDL-C and VLDL-C } \\
\downarrow \text { Lipoprotein (a) } \\
\text { Transforms LDL-C to } \\
\text { less atherogenic form by } \\
\text { increasing average particle } \\
\text { size and also decreases LDL } \\
\text { particle concentration }\end{array}$ & $\begin{array}{l}\text { Potential for frequent skin } \\
\text { flushing, pruritus, abdominal } \\
\text { discomfort, hepatotoxicity (rare } \\
\text { but may be severe), nausea, } \\
\text { peptic ulcer, atrial fibrillation } \\
\text { Deleterious effect on serum } \\
\text { glucose at higher dosages } \\
\text { Increases uric acid levels; may } \\
\text { lead to gout }\end{array}$ \\
\hline
\end{tabular}

LDL-C: low-density lipoprotein cholesterol; TG: triglycerides; HDL-C: highdensity lipoprotein cholesterol; VLDL-C: very low-density lipoprotein cholesterol.

Nicotinic acid has broad lipid-modulating action, raising HDL-C in a dosedependent manner by up to $25 \%$ and reducing LDL-C by $15-18 \%$ and TGs by $20-40 \%$ at the $2 \mathrm{~g} /$ day dose. Nicotinic acid is unique in lowering lipoprotein(a) (Lp(a)) levels by up to $30 \%$ at this dose. After two large studies with nicotinic acid, one with extended-release niacin [127] and one with niacin plus laropiprant [128], showed no beneficial effect, but rather an increased frequency of serious adverse effects, no medication containing nicotinic acid is currently approved in Europe [115].

In summary, nicotinic acid [117]:

$\downarrow$ LDL-C $10-25 \%$;

$\downarrow$ TG $20-30 \%$;

$\uparrow$ HDL-C $10-35 \%$ by decreasing hepatic synthesis of LDL-C and VLDL-C; $\downarrow \mathrm{LP}(\mathrm{a})$.

Transforms LDL-C to less atherogenic form by increasing average particle size and also decreases LDL particle concentration.

Nicotinic acid (immediate-release). Usual recommended starting daily dosage $250 \mathrm{mg}$; dosage range $250-3.000 \mathrm{mg}$.

Nicotinic acid (extended-release). Usual recommended starting daily dosage $500 \mathrm{mg}$; dosage range 500-2.000 $\mathrm{mg}$ [117].

\subsubsection{Bile acid sequestrants}

Bile acids are synthesized in the liver from cholesterol and are released into the intestinal lumen, but most of the bile acid is returned to the liver from the terminal ileum via active absorption. The two older bile acid sequestrants, 
cholestyramine and colestipol, are both bile acid-binding exchange resins. Recently the synthetic drug colesevelam was introduced.

Information about the bile acid sequestrants: Metabolic effect and main considerations are given in Table 15. [117]

Table 15 Bile acid sequestrants: Metabolic effect and main considerations

\begin{tabular}{|c|c|c|}
\hline Drug class & Metabolic effect & Main considerations \\
\hline $\begin{array}{l}\text { Bile acid sequestrants } \\
\text { (cholestyramine, } \\
\text { colestipol, colesevelam } \\
\text { hydrochloride) }\end{array}$ & $\begin{array}{l}\text { Primary } \downarrow \text { LDL-C } 15-25 \% \\
\text { by binding bile acids and } \\
\text { preventing their reabsorption } \\
\text { in the ileum (causing hepatic } \\
\text { cholesterol depletion and } \\
\text { LDLR upregulation) } \\
\text { Colesevelam } \downarrow \text { glucose and } \\
\text { HbA1c }(\sim 0.5 \%) \text {; is FDA } \\
\text { approved to treat T2DM }\end{array}$ & $\begin{array}{l}\text { May } \uparrow \text { serum TG } \\
\text { Frequent constipation and/ } \\
\text { or bloating, which can reduce } \\
\text { adherence } \\
\text { Many potential drug } \\
\text { interactions (decreased drug } \\
\text { absorption), less so with } \\
\text { colesevelam (see product } \\
\text { labeling) } \\
\text { May reduce absorption of folic } \\
\text { acid and fat-soluble vitamins } \\
\text { such as vitamins A, D, and K }\end{array}$ \\
\hline
\end{tabular}

LDL-C: low-density lipoprotein cholesterol; LDLR: low-density lipoprotein receptor; HbAlc: glycated hemoglobin A1c; FDA: U.S. Food and Drug Administration; T2DM: type 2 diabetes mellitus; TG: triglycerides.

The bile acid sequestrants are not systemically absorbed or altered by digestive enzymes, therefore the beneficial clinical effects are indirect. By binding the bile acids, the drugs prevent the entry of bile acids into the blood and thereby remove a large portion of the bile acids from the enterohepatic circulation. The increase in cholesterol catabolism to bile acids results in a compensatory increase in hepatic LDLR activity, clearing LDL-C from the circulation and thus reducing LDL-C levels. These agents also reduce glucose levels in hyperglycemic patients [115, 129].

In summary, bile acid sequestrants [117]:

$\downarrow$ LDL-C (primarily) $15-25 \%$ by binding bile acids and preventing their reabsorption in the ileum (causing hepatic cholesterol depletion and LDLR upregulation);

$\downarrow$ glucose and $\mathrm{HbA}_{1 \mathrm{c}}(\sim 0.5 \%)$ (colesevelam); is FDA approved to treat T2DM.

Cholestyramine. Usual recommended starting daily dosage 8-16 g; dosage range 4-24 g. Colestipol. Usual recommended starting daily dosage $2 \mathrm{~g}$; dosage range $2-16 \mathrm{~g}$.

Colesevelam. Usual recommended starting daily dosage $3.8 \mathrm{~g}$; dosage range 3.8-4.5 g.

Combination therapies (single pill). Ezetimibe/simvastatin. Usual recommended starting daily dosage 10/20 mg; dosage range 10/10-10/80 mg. Extended-release niacin/simvastatin. Usual recommended starting daily dosage $500 / 20 \mathrm{mg}$; dosage range 500/20-1.000/20 $\mathrm{mg}$ [117]. 


\subsubsection{Inhibitors of microsomal triglycerides transfer protein}

Within the lumen of the endoplasmic reticulum, lomitapide inhibits microsomal TG transfer protein (MTP), which prevents the formation of apoB, and, thus, the formation of VLDL and chylomicrons as well. Altogether, this leads to a reduction of LDL-C. Lomitapide, the MTP inhibitor, and mipomersen, the antisense oligonucleotides against apo B, have shown their efficacy in lowering LDL-C in recent phase III trials and they were already approved for treating patients with homozygous familial hypercholesterolemia [130].

In summary, MTP inhibitor [117]:

$\downarrow$ Up to LDL-C $40 \%$, TC $36 \%$, apoB $39 \%$, TG 45\%, and non- HDL-C $40 \%$ (depending on dose) in patients with homozygous familial hypercholesterolemia by binding and inhibiting MTP, which inhibits synthesis of chylomicrons and VLDL.

Lomitapide. Usual recommended starting daily dosage $5 \mathrm{mg}$, with subsequent titration; dosage range 5-60 $\mathrm{mg}$ [117].

Information about the MTP inhibitor (lomitapide): Metabolic effect and main considerations are given in Table 16.

Table 16 MTP inhibitor (lomitapide): Metabolic effect and main considerations [117]

\begin{tabular}{|c|c|c|}
\hline Drug class & Metabolic effect & Main considerations \\
\hline $\begin{array}{l}\text { MTP inhibitor } \\
\text { (lomitapide) }\end{array}$ & $\begin{array}{l}\downarrow \text { Up to LDL-C } 40 \%, \text { TC } \\
36 \% \text {, apo B } 39 \%, \text { TG } \\
45 \% \text {, and non- HDL-C } \\
40 \% \text { (depending on dose) } \\
\text { in patients with HoFH } \\
\text { by binding and inhibiting } \\
\text { MTP, which inhibits } \\
\text { synthesis of chylomicrons } \\
\text { and VLDL }\end{array}$ & $\begin{array}{l}\text { Can cause increases in transaminases } \\
\text { (ALT, AST). Monitoring of ALT, } \\
\text { AST, alkaline phosphatase, and total } \\
\text { bilirubin prior to initiation, and of } \\
\text { ALT and AST during treatment, is } \\
\text { required per FDA REMS } \\
\text { Causes increases in hepatic } \\
\text { fat (steatosis) with or without } \\
\text { concomitant elevated transaminases, } \\
\text { which may be a risk for progressive } \\
\text { liver diseases } \\
\text { Also causes steatosis of the small } \\
\text { intestine with resulting abdominal } \\
\text { pain and steatorrhea unless a very- } \\
\text { low-fat diet is followed. May also } \\
\text { cause fat-soluble vitamin deficiency } \\
\text { unless vitamin supplements are taken } \\
\text { Caution should be exercised } \\
\text { when used with other drugs with } \\
\text { potential hepatotoxicity. Because of } \\
\text { hepatotoxicity risk, only available } \\
\text { through REMS program }\end{array}$ \\
\hline
\end{tabular}

LDL-C: low-density lipoprotein cholesterol; Apo: apolipoprotein; HDL-C: highdensity lipoprotein cholesterol; HoFH: homozygous familial hypercholesterolemia; MTP: microsomal triglyceride transfer protein; VLDL-C: very low-density lipoprotein 
cholesterol; FDA: U.S. Food and Drug Administration; REMS: Risk Evaluation and Mitigation Strategies.

\subsubsection{Antisense apolipoprotein B oligonucleotide (mipomersen via subQ injection)}

Mipomersen is a second-generation antisense oligonucleotide (ASO) targeted to human apoB-100, large protein synthesized by the liver that plays a fundamental role in human lipoprotein metabolism. Mipomersen predominantly distributes to the liver and decreases the production of apoB-100, the primary structural protein of the atherogenic lipoproteins including LDL, thereby reducing plasma LDL-C and apoB-100 concentrations [131].

Information about the antisense apolipoprotein B oligonucleotide: Metabolic effect and main considerations are given in Table 17.

Table 17 Antisense apo B oligonucleotide: Metabolic effect and main considerations [117]

\begin{tabular}{|l|l|l|}
\hline \multicolumn{1}{|c|}{ Drug class } & \multicolumn{1}{|c|}{ Metabolic effect } & \multicolumn{1}{c|}{ Main considerations } \\
\hline $\begin{array}{l}\text { Antiseptive apolipoprotein B } \\
\text { oligonucleotide (mipomersen } \\
\text { via subQ injection) }\end{array}$ & $\begin{array}{l}\downarrow \text { LDL-C 21\%, TC 19\%, } \\
\text { apo B 24\%, and non- } \\
\text { HDL-C 22\% in patients } \\
\text { with HoFH by degrading } \\
\text { mRNA for apo B-100, the } \\
\text { principal apolipoprotein } \\
\text { needed for hepatic } \\
\text { synthesis of VLDL (and } \\
\text { subsequent intraplasma } \\
\text { production of HDL and } \\
\text { LDL) }\end{array}$ & $\begin{array}{l}\text { Can cause increases in } \\
\text { transaminases (ALT, AST). } \\
\text { Monitoring of ALT, AST, } \\
\text { alkaline phosphatase, and } \\
\text { total bilirubin prior to } \\
\text { initiation, and of ALT and } \\
\text { AST during treatment is } \\
\text { recommended } \\
\text { Causes increases in hepatic } \\
\text { fat (steatosis) with or without } \\
\text { concomitant elevated } \\
\text { transaminases, which may be } \\
\text { a risk for progressive liver } \\
\text { diseases } \\
\text { Caution should be exercised } \\
\text { when used with other drugs } \\
\text { with potential hepatotoxicity. } \\
\text { Because of hepatotoxicity } \\
\text { risk, only available through } \\
\text { REMS program }\end{array}$ \\
& & \\
\end{tabular}

LDL-C: low-density lipoprotein cholesterol; TC: total cholesterol; HDL-C: high-density lipoprotein cholesterol; HoFH: homozygous familial hypercholesterolemia; Apo: apolipoprotein; REMS: Risk Evaluation and Mitigation Strategies.

In summary, antisense apo B oligonucleotide [117]:

$\downarrow$ LDL-C $21 \%$, TC $19 \%$, apo B $24 \%$, and non-HDL-C $22 \%$ in patients with HoFH by degrading mRNA for apoB-100, the principal apolipoprotein needed for hepatic synthesis of VLDL (and subsequent intraplasma production of LDL). 
Mipomersen (SubQ injection). Usual recommended starting daily dosage 200 $\mathrm{mg}$ once weekly, with subsequent titration; dosage range $200 \mathrm{mg}$ once weekly [117].

\subsubsection{Omega-3 fatty acids}

Omega-3 PUFAs (eicosapentaenoic acid and docosahexaenoic acid) are used at pharmacological doses to lower TGs. Omega-3 fatty acids (2-4 g/day) affect serum lipids and lipoproteins, in particular VLDL concentration. The underlying mechanism is poorly understood, although it may be related, at least in part, to their ability to interact with PPARs and to a decreased secretion of apoB [115].

Information about the antisense apolipoprotein B oligonucleotide: Metabolic effect and main considerations are given in Table $\mathbf{1 8 .}$

Table 18 Omega-3 fatty acids: Metabolic effect and main considerations [117]

\begin{tabular}{|c|c|c|}
\hline Drug class & Metabolic effect & Main considerations \\
\hline $\begin{array}{l}\text { Omega-3 fatty } \\
\text { acids (icosapent } \\
\text { ethyl, omega-3-acid } \\
\text { ethyl esters) }\end{array}$ & $\begin{array}{l}\text { TG } \downarrow 27-45 \% \text {, TC } \downarrow \\
7-10 \%, \downarrow \text { VLDL-C } \\
20-42 \% \text {, apo B } 4 \%, \\
\text { and non- HDL-C } \\
8-14 \% \text { in individuals } \\
\text { with severe } \\
\text { hypertriglyceridemia, } \\
\text { most likely by } \\
\text { reducing hepatic } \\
\text { VLDL-TG synthesis } \\
\text { and/or secretion } \\
\text { and enhancing } \\
\text { TG clearance } \\
\text { from circulating } \\
\text { VLDL particles. } \\
\text { Other potential } \\
\text { mechanisms of action } \\
\text { include: increased } \\
\beta \text {-oxidation; } \\
\text { inhibition of } \\
\text { acyl-CoA; } \\
1,2 \text {-diacylglyceral } \\
\text { acyltransferase; } \\
\text { decreased hepatic } \\
\text { lipogenesis; and } \\
\text { increased plasma } \\
\text { lipoprotein activity } \\
\text { Icosapent ethyl } \downarrow \\
\text { LDL-C 5\%, whereas } \\
\text { omega-3-acid ethyl } \\
\text { esters } \uparrow \text { LDL-C } 45 \%\end{array}$ & $\begin{array}{l}\text { TG levels should be carefully assessed prior } \\
\text { to initiating therapy and periodically during } \\
\text { therapy } \\
\text { Omega3-acid ethyl esters can increase } \\
\text { LDL-C levels. Monitoring of LDL-C levels } \\
\text { during treatment is recommended } \\
\text { May prolong bleeding time. Periodic } \\
\text { monitoring of coagulation status should be } \\
\text { undertaken in patients receiving treatment } \\
\text { with omega-3 fatty acids and other drugs } \\
\text { affecting coagulation } \\
\text { Periodic monitoring of ALT and AST levels } \\
\text { during treatment is recommended for patients } \\
\text { with hepatic impairment. Some patients may } \\
\text { experience increases in ALT levels only } \\
\text { Caution should be exercised when treating } \\
\text { patients with a known hypersensitivity to fish } \\
\text { and/or shellfish } \\
\text { The effect of omega-3 fatty acids on } \\
\text { cardiovascular morbidity and mortality } \\
\text { and the risk of pancreatitis has not } \\
\text { been determined in patients with severe } \\
\text { hypertriglyceridemia } \\
\text { In patients with paroxysmal or persistent AF, } \\
\text { therapy with omega-3-acid ethyl esters may } \\
\text { be associated with increased frequency of } \\
\text { symptomatic AF or flutter, especially within } \\
\text { the first } 2 \text { to } 3 \text { months after initiation } \\
\text { The most common adverse events in patients } \\
\text { receiving omega-3 fatty acids included } \\
\text { arthralgia ( } 2.3 \%) \text {, eructation ( } 4 \% \text { ), dyspepsia } \\
\text { (3\%), and taste perversion ( } 4 \% \text { ). }\end{array}$ \\
\hline
\end{tabular}




\begin{tabular}{|l|l|}
\hline & $\begin{array}{l}\text { Patients may also experience constipation, } \\
\text { gastrointestinal disorders, vomiting, rash, or } \\
\text { pruritus } \\
\text { Omega-3 fatty acids should be used with } \\
\text { caution in nursing mothers and should only } \\
\text { be used in pregnant women if the benefits of } \\
\text { treatment outweigh the potential risk of fetal } \\
\text { harm }\end{array}$ \\
\hline
\end{tabular}

TG: triglycerides; TC: total cholesterol; VLDL-C: very low-density lipoprotein cholesterol; LDL-C: low-density lipoprotein cholesterol; HDL-C: high-density lipoprotein cholesterol; AF: atrial fibrillation.

In summary, omega-3 fatty acids [117]:

$\downarrow$ TG $27-45 \%$, TC 7-10\%, VLDL-C 20-42\%, apoB 4\%, and non-HDL-C $8-14 \%$ in individuals with severe hypertriglyceridemia, most likely by reducing hepatic VLDL-TG synthesis and/or secretion and enhancing TG clearance from circulating VLDL particles.

Other potential mechanisms of action include [117]:

$\uparrow \beta$-oxidation;

$\downarrow$ inhibition of acyl-CoA; 1,2-diacylglyceral acyltransferase;

$\downarrow$ decreased hepatic lipogenesis;

$\uparrow$ increased plasma lipoprotein activity;

$\downarrow$ LDL-C 5\% (Icosapent ethyl);

$\uparrow$ LDL-C $45 \%$ (omega-3-acid ethyl esters).

Omega-3-acid ethyl esters (Lovaza). Usual recommended starting daily dosage 4 g per day; dosage range $4 \mathrm{~g}$ per day.

Icosapent ethyl (Vascepa $\left.{ }^{\circledR}\right)$ Usual recommended starting daily dosage 4 g per day; dosage range $4 \mathrm{~g}$ per day [117].

Information about the lipid-lowering drug therapies, usual starting dosages and dosage ranges are given in Table 19.

Table 19 Lipid-lowering drug therapies, usual starting dosages and dosage ranges [117]

\begin{tabular}{|l|c|c|}
\hline \multicolumn{1}{|c|}{ Agent } & $\begin{array}{c}\text { Usual recommended starting } \\
\text { daily dosage }\end{array}$ & Dosage range \\
\hline Statins & & $10-80 \mathrm{mg}$ \\
\hline Lovastatin & $20 \mathrm{mg}$ & $10-80 \mathrm{mg}$ \\
\hline Pravastatin & $40 \mathrm{mg}$ & $5-80 \mathrm{mga}$ \\
\hline Simvastatin & $20-40 \mathrm{mg}$ & $20-80 \mathrm{mg}$ \\
\hline Fluvastatin & $40 \mathrm{mg}$ & $10-80 \mathrm{mg}$ \\
\hline Atorvastatin & $10-20 \mathrm{mg}$ & $5-40 \mathrm{mg}$ \\
\hline Rosuvastatin & $10 \mathrm{mg}$ & $2-4 \mathrm{mg}$ \\
\hline Pitavastatin & $2 \mathrm{mg}$ & \\
\hline
\end{tabular}


Diabetic cardiovascular neuropathy

\begin{tabular}{|c|c|c|}
\hline $\begin{array}{l}\text { Cholesterol absorption } \\
\text { inhibitors }\end{array}$ & & \\
\hline Ezetimibe & $10 \mathrm{mg}$ & $10 \mathrm{mg}$ \\
\hline \multicolumn{3}{|l|}{ PCSK9 Inhibitors } \\
\hline Alirocumab & $75 \mathrm{mg}$ every 2 weeks & $75-150 \mathrm{mg}$ every 2 weeks \\
\hline Evolocumab & $\begin{array}{l}140 \mathrm{mg} \text { every } 2 \text { weeks or } \\
420 \mathrm{mg} \text { once monthly }\end{array}$ & Not applicable \\
\hline \multicolumn{3}{|l|}{ Fibrates } \\
\hline Fenofibrate & $48-145 \mathrm{mg}$ & $48-145 \mathrm{mg}$ \\
\hline Gemfibrozil & $1.200 \mathrm{mg}$ & $1.200 \mathrm{mg}$ \\
\hline Fenofibric acid & $45-135 \mathrm{mg}$ & $45-135 \mathrm{mg}$ \\
\hline \multicolumn{3}{|l|}{ Niacin } \\
\hline Immediate-release & $250 \mathrm{mg}$ & $250-3.000 \mathrm{mg}$ \\
\hline Extended-release & $500 \mathrm{mg}$ & $500-2.000 \mathrm{mg}$ \\
\hline \multicolumn{3}{|l|}{ Bile acid sequestrants } \\
\hline Cholestyramine & $8-16 \mathrm{~g}$ & $4-24 \mathrm{~g}$ \\
\hline Colestipol & $2 \mathrm{~g}$ & $2-16 \mathrm{~g}$ \\
\hline Colesevelam & $3.8 \mathrm{~g}$ & $3.8-4.5 \mathrm{~g}$ \\
\hline \multicolumn{3}{|l|}{$\begin{array}{l}\text { Combination therapies } \\
\text { (single pill) }\end{array}$} \\
\hline Ezetimibe/simvastatin & $10 / 20 \mathrm{mg}$ & $10 / 10-10 / 80 \mathrm{mg}$ \\
\hline $\begin{array}{l}\text { Extended-release niacin/ } \\
\text { simvastatin }\end{array}$ & $500 / 20 \mathrm{mg}$ & $500 / 20-1.000 / 20 \mathrm{mg}$ \\
\hline \multicolumn{3}{|l|}{ MTP inhibitor } \\
\hline Lomitapide & $\begin{array}{l}5 \mathrm{mg} \text {, with subsequent } \\
\text { titration }\end{array}$ & $5-60 \mathrm{mg}$ \\
\hline \multicolumn{3}{|l|}{$\begin{array}{l}\text { Antisense apolipoprotein B } \\
\text { oligonucleotide }\end{array}$} \\
\hline $\begin{array}{l}\text { Mipomersen (SubQ } \\
\text { injection) }\end{array}$ & $200 \mathrm{mg}$ once weekly & $200 \mathrm{mg}$ once weekly \\
\hline \multicolumn{3}{|l|}{ Omega-3 fatty acids } \\
\hline $\begin{array}{l}\text { Omega-3-acid ethyl esters } \\
\text { (Lovaza) }\end{array}$ & 4 g per day & 4 g per day \\
\hline Icosapent ethyl (Vascepa $\left.{ }^{\circledR}\right)$ & 4 g per day & 4 g per day \\
\hline
\end{tabular}


MTP: Microsomal transfer protein; PCSK9: Proprotein convertase subtilisin/ kexin type 9; SubQ: Subcutaneous.

\subsection{Specific features of dyslipidaemia in insulin resistance and type 2 diabetes mellitus}

Diabetic DLP is a cluster of plasma lipid and lipoprotein abnormalities that are metabolically interrelated. The increase in large VLDL particles in T2DM initiates a sequence of events that generates atherogenic remnants, small dense LDL and small TG-rich dense HDL particles [115, 132]. These components are not isolated abnormalities but are closely linked to each other. Both LDL and HDL particles show variable compositional changes that are reflected in their functions. Notably apoCIII levels are increased in subjects with T2DM. Together, triglyceride-rich lipoproteins remnants, small dense LDL and small dense HDL comprise the atherogenic lipid profile, which is also characterized by an increase in apoB concentration due to an increased number of apoB-containing particles $[115,133,134]$.

\subsection{Evidence for low-density lipoprotein-lowering therapy}

LDL-C is stipulated to be the primary target of lipid-lowering therapy in DM. Trials specifically performed in subjects with T2DM as well as subsets of individuals with DM in major statin trials have consistently demonstrated significant benefits of statin therapy on CVD events in people with T2DM. Statin therapy reduces the 5-year incidence of major CVD events by $23 \%$ per $1 \mathrm{mmol} / \mathrm{L}$ reduction in LDL-C, regardless of the initial LDL-C or other baseline characteristics based on meta-analysis. The Cholesterol Treatment Trialists'meta-analysis further indicates that subjects with T2DM will have a relative risk reduction that is comparable to that seen in non-diabetic patients, but being at higher absolute risk, the absolute benefit will be greater, resulting in a lower number needed to treat $[115,135]$. Recent studies have suggested an increased incidence of diabetes in patients treated with statins [136]. This effect must not lessen our attention to the treatment of patients, as the overall benefit in cardiovascular events reduction remains [115].

\subsection{Triglycerides and high-density lipoprotein cholesterol}

Clinical benefits achieved by the treatment of atherogenic DLP (high TGs and low HDL-C) are still a matter of discussion. Although the Helsinki Heart Study reported a significant reduction in CVD outcomes with gemfibrozil, neither the Fenofibrate Intervention and Event Lowering in Diabetes (FIELD) nor the ACCORD study showed a reduction in total CVD outcomes [115, 137, 138]. In a post hoc analysis of the FIELD study, fenofibrate reduced CVD events by $27 \%$ in those with elevated TGs $[>2.3 \mathrm{mmol} / \mathrm{L}(\sim 204 \mathrm{mg} / \mathrm{dL})]$ and reduced HDL-C $[115,133]$. Consistent with these findings, a meta-analysis of fibrates in the prevention of CVD in people with T2DM showed that fibrates significantly reduced the risk of non-fatal MI by $21 \%$, but had no effect on the risk of overall mortality or coronary mortality [139]. 


\subsection{Treatment strategies for patients with type 2 diabetes mellitus and metabolic syndrome}

Lifestyle therapy to improve the atherogenic lipid profile should be recommended to all subjects with T2DM and MetS. Dietary advice should be tailored according to the individual's needs. If LDL-C goals are not achieved on maximally tolerated doses of statins, drug combinations may offer additional lowering of LDL-C, but the evidence from outcome studies is limited [115]. Patients with T2DM $<40$ years of age, with a short duration of therapy, without other risk factors, without complications and with an LDL-C level $<2.6 \mathrm{mmol} / \mathrm{L}(<100 \mathrm{mg} / \mathrm{dL})$ may not need lipid-lowering drugs [115].

T1DM. The lipid profile in T1DM subjects with good glycemic control is "supernormal"and characterized by subnormal TGs and LDL-C, whereas HDL-C is usually within the upper normal range or slightly elevated. However, there are potentially atherogenic changes in the composition of both HDL and LDL particles. In all patients with T1DM and in the presence of microalbuminuria and renal disease, LDL-C lowering (at least 30\%) with statins as the first choice (drug combination may be considered if needed) is recommended irrespective of the basal LDL-C concentration [115]. Recommendations for the treatment of DLP in DM [15]: in all patients with T1DM and in the presence of microalbuminuria and/ or renal disease, LDL-C lowering (at least 50\%) with statins as the first choice is recommended irrespective of the baseline LDL-C concentration (class I, level C) $[115,140,141]$; in patients with T2DM and CVD or chronic kidney disease, and in those without CVD who are $>40$ years of age with one or more other CVD risk factors or markers of target organ damage, the recommended goal for LDL-C is $<1.8 \mathrm{mmol} / \mathrm{L}(<70 \mathrm{mg} / \mathrm{dL})$ and the secondary goal for non-HDL-C is $<2.6 \mathrm{mmol} / \mathrm{L}(<100 \mathrm{mg} / \mathrm{dL})$ and for apoB is $<80 \mathrm{mg} / \mathrm{dL}$ (class I, level B) [135, $141]$.

In all patients with T2DM and no additional risk factors and/or evidence of target organ damage, LDL-C $<2.6 \mathrm{mmol} / \mathrm{L}(<100 \mathrm{mg} / \mathrm{dL})$ is the primary goal. Non-HDL-C $<3.4 \mathrm{mmol} / \mathrm{L}(<130 \mathrm{mg} / \mathrm{dL})$ and apoB $<100 \mathrm{mg} / \mathrm{dL}$ are the secondary goals (class I, level B) $[135,141]$.

\subsection{Fatty acids metabolism disorders ( $\gamma$-linolenic acid, acetyl L-carnitine)}

Vasoactive prostanoids, metabolites and DGLA, including prostaglandins and other eicosanoids are necessary for the physiological behavior of nerve conductivity and blood flow. The results of double-blind, placebo-controlled studies showed that prescription of DGLA to patients with DPN is followed by positive dynamics in clinical course, as well as increase in the speed of nerve conductivity. L-carnitine's main function is to strengthen the metabolism of fatty acids, but there are experimental evidence of L-carnitine's ability to activate glucose metabolism. It is believed that T2DM is characterized by malfunction of L-carnitine exchange in the mitochondria. The results of several studies showed that prescription of L-carnitine helps to improve energy supplies and LV function. It is established that propionyl-L-carnitine improves the functional status, used as glucose energy oxidation in the rat's affected myocardium (despite the increased level of fatty acids). Nutrition of diabetic mice with obesity with L-carnitine addition increases the level of acyl-carnitine in the blood, muscle, liver and adipose 
tissue; increases levels of pyruvate dehydrogenase activity in the muscles; prescription of zinc-carnitine mixture reduces hyperglycemia and improves glucose tolerance. L-carnitine infusion with the help of hyperinsulinemic-euglycemic clamp improves glucose profile control, reduces the concentration of circulating lipids. L-carnitine prescription for 3 or 6 mo for newly diagnosed patients with T2DM with lipid metabolism disorders is followed by a statistically significant decrease in $\mathrm{Lp}$ (a) levels. The results of double-blind, placebo-controlled studies among patients with verified hyperLP(a) established that L-carnitine $(2 \mathrm{~g} / \mathrm{d})$ encouraged a significant decrease in the concentration of $\mathrm{Lp}(\mathrm{a})$ levels; L-carnitine incorporation into nutrition of patients with newly diagnosed T2DM is followed by similar changes; combined L-carnitine with simvastatin $(20 \mathrm{mg} / \mathrm{d})$ treatment is much more efficient in decreasing the concentration of lipids, including TG and $\mathrm{Lp}$ (a) than statin monotherapy. Thus, L-carnitine can be used as one of the components for lipid-modifying therapy among patients with T2DM [142, 143].

\subsection{Antioxidant therapy}

Increasing evidence suggests that OS plays a role in the pathogenesis of DM and its complications. Hyperglycemia increases OS, which contributes to the impairment of the main processes that fail during $\mathrm{DM}$, insulin action and insulin secretion. In addition, antioxidant (AO) mechanisms are diminished in diabetic patients, which may further augment OS. Hyperglycemia-induced OS and nitrosative stress has been singled out as one of the major links between DM and diabetic complications; leads to generation of free radicals due to autoxidation of glucose and glycosylation of proteins $[144,145]$. The persistent increase in reactive oxygen species (ROS) and reactive nitrogen species (RNS) accompanied by a decrease in $\mathrm{AO}$ activity leads to the occurrence of OS and nitrosative stress which can cause endothelial dysfunction, IR, and eventually leads to diabetic microvascular and macrovascular complications [146]. Reactive species can be eliminated by a number of enzymatic and nonenzymatic antioxidant mechanisms. Superoxide dismutase (SOD) immediately converts $\cdot \mathrm{O}_{2}$ - to hydrogen peroxide $\left(\mathrm{H}_{2} \mathrm{O}_{2}\right)$, which is then detoxified to water either by catalase in the lysosomes or by glutathione peroxidase (GPx) in the mitochondria. Another enzyme that is important is glutathione reductase (GSR), which regenerates glutathione that is used as a hydrogen donor by GPx during the elimination of $\mathrm{H}_{2} \mathrm{O}_{2}$. Thus, modulation of these enzymes in target organs prone to diabetic complications such as heart may prove beneficial in the prevention and management of heart failure [144, 145].

Hyperlipidemia in the presence of hyperglycemia generates additional ROS that are also implicated in cell dysfunction $[145,147]$. OS has been implicated in causing nerve damage in several animal, human, and experimental models of diabetes $[145,148]$. The mechanisms involved in OS-induced nerve dysfunctions include generation of ROS, increased RNS, lipid peroxidation (LPO), deoxyribonucleic acid (DNA) damage, and reduction in cellular antioxidants [145, 149]. Increased ROS and RNS together with reductions in the AO defense mechanisms within the neurons contribute to the manifestations of DPN which include nerve blood flow impairment, endoneurial hypoxia, nerve degeneration, axonal atrophy. Recent findings implicate free radicals in the development of $\mathrm{DN}$ in addition to the impairment of AO defense system in T2DM [144]. 
Also, induction of aldose reductase enzyme depletes the reduced form of nicotinamide adenine dinucleotide phosphate (NADPH), a requirement for the regeneration of the cellular AO, reduced glutathione (GSH), contributing to OS $[7,145,150,151]$. Itra- and intermolecular cross-linking reactions with proteins, lipids, or DNA lead to the formation of stable, covalent, and irreversible adducts collectively referred to as advanced glucose end-products (AGEs) that accumulate within cells with age $[145,150]$. Increased formation of AGEs leads to the elevation of OS and subsequently damage to cells and tissues, an occurrence that has been found in experimental animals and in humans [152]. AGEs have also been shown to decrease axonal transport within neurons leading to their degeneration [145, 153].

Antioxidants are available endogenously as a physiological defense mechanism of the cell or obtained exogenously from diet. The enzymatic AO systems, such as copper, zinc, manganese and selenium, SOD, GPx, GSR, and catalase may remove the ROS directly or sequentially, preventing their excessive accumulation and consequent adverse effects. Non-enzymatic AO systems consist of scavenging molecules that are endogenously produced such as GSH, ubichinol, and uric acid or derivatives of the diet such as vitamins A, C and E, carotenoids, lipoic acid (LA); coenzyme $\mathrm{Q}_{10}\left(\mathrm{CoQ}_{10}\right)$; and cofactors like folic acid, uric acid, albumin, and vitamins $\mathrm{B}_{1}, \mathrm{~B}_{3}[154,155]$. Vitamins $\mathrm{C}, \mathrm{E}$ and LA are involved in the termination of the LPO process ${ }^{[154]}$. The abilities of flavonoids to scavenge free radicals have also been reported [145].

However, in the case of macrovascular/microvascular complications, the antioxidant therapy is beneficial together with BP control, management of atherogenic DLP, and optimal glucose control $[145,155,156]$.

9.10.1. Strategies targeted directly against reactive oxygen species and reactive nitrogen species [145]

Diabetes-induced nerve dysfunction is established to be caused by an increase in the overproduction of ROS and RNS. It was therefore hypothesized that antioxidants or agents that directly scavenge free radicals can reduce the formation or progression of ROS reactions which in turn decreases OS thereby improving DN conditions [145]. Some of the most important antioxidants include ALA, vitamins $\mathrm{A}, \mathrm{C}$, and $\mathrm{E}$, acetyl L-carnitine, taurine, and melatonin.

\subsubsection{Alpha-lipoic acid}

ALA has gained a considerable amount of attention as an antioxidant. ALA can be biosynthesized in plants and animals where it is metabolized to dihydrolipoic acid (DHLA) upon uptake into cells. Both ALA and DHLA are potent free radical scavengers that are also involved in the regeneration of vitamins $\mathrm{C}$ and $\mathrm{E}$ and oxidized GSH within the cell [157]. ALA is also a cofactor for a number of mitochondrial enzymes [145]. ALA is known to reduce OS by inhibiting hexosamine and AGEs pathways [145].

Alpha-lipoic acid, a critical co-factor for mitochondrial dehydrogenase reactions, is another compound with free radical-scavenging activity [158]. ALA was found to increase glucose transport in muscle cells in culture by stimulating translocation of glucose transporter type 4 (GLUT4) from internal pools to the plasma membrane 
[155]. Mechanism of ALA action is not fully developed, but specific attention should be paid to two hypotheses. Firstly, ALA phenomenon causes dose-dependent proliferation of neuroblastoma cultured cells. Changes in the membrane fluidity that are mediated through sulfhydryl groups ALA are considered to cause this effect. This is confirmed by the following results of several studies, including experimental neuropathy induced by acrylamide, followed by a significant inhibition of proliferation of the above phenomenon; overlay and/or progression of experimental distal neuropathy, mainly caused by a decrease of content of substances in axons containing sulfhydryl groups (e.g., GSH); ALA in vivo and in vitro enhances spontaneous processes of expansion and improvement of the structural and functional nerve terminals membranes state; prescription of ALA stimulates the regeneration of nerve terminals in case of the partial denervation, as well as experimental hexacarbon neuropathy. Secondly, and the most probable mechanism is the ability of ALA to function as a radical binder ("cleaner") [89, 155, 159].

In cultured adipocytes, treatment with ALA protected the insulin receptor from oxidative damage, maintaining its functional integrity. A placebo-controlled explorative study of patients with T2DM indicated that oral administration of ALA significantly increased insulin-mediated glucose uptake, presumably by modulating insulin sensitivity [155]. ALA600SOD (an oral formulation of ALA and SOD) improved symptoms and electroneurographic parameters among subjects with DPN [160].

\subsubsection{Vitamins A, B1, B3, C and E}

Dietary antioxidant vitamins such as vitamins $\mathrm{A}, \mathrm{C}$, and $\mathrm{E}$ detoxify free radicals directly and also interact with recycling processes to create reduced forms of the vitamins. Antioxidant vitamins have a number of biological activities such as immune stimulation and prevention of genetic changes by inhibiting DNA damage induced by the ROS metabolites [161].

\subsection{Vitamin A}

Vitamin A has a plethora of cellular actions. Besides modulating gene expression, cell growth and differentiation, this vitamin may also act as AO, although the mechanisms of action in this role are not fully deciphered [161]. The AO potential of carotenoids (vitamin A) depends on their distinct membrane-lipid interactions, while some carotenoids can decrease LPO, others can stimulate it [161].

\subsection{Vitamin $B_{1}$}

Thiamine derivatives are cofactor for enzymes involved in the production of chemical energy from carbohydrates and fat. Thiamine deficiency (TD) may be associated with specific and selective neuronal cell death and damage of the bloodbrain barrier. DM might be considered as TD state, if not in absolute terms at least relative to the increased requirements deriving from accelerated and amplified glucose metabolism in non-insulin dependent tissues that, like the vessel wall, are prone to complications. The TD in clinical diabetes may increase the fragility of vascular cells to the adverse effects of hyperglycemia and there by the increase of the risk of developing microvascular complications [162]. 


\subsection{Nicotinamide (Vitamin $\mathrm{B}_{3}$ )}

The vitamin plays an important role in mitochondrial energy generation and DNA repair. Deficiency of nicotinamide is associated with dermatological, gastrointestinal, hematological and nervous system dysfunction. Sensory neuropathy due to vitamin $\mathrm{B}_{3}$ deficiency is characterized by decreased sensation to touch and vibration [156].

\subsection{Vitamin C}

Ascorbic acid serves as a cofactor for hydroxylation and function of monooxygenase enzymes in the synthesis of sub-tissues (collagen), neurotransmitters and carnitine. Ascorbic acid is an antioxidant acting as an enzymatic cofactor in maintaining tissue integrity and plays an important role in formation of epithelial and endothelial barriers and aids in regeneration of oxidized vitamin $\mathrm{E}$ [156].

Vitamin $\mathrm{C}$ has a role in scavenging ROS and RNS by becoming oxidated itself. The oxidized products of vitamin $\mathrm{C}$, ascorbic radical and dehydroascorbic radical are regenerated by $\mathrm{GSH}$, the reduced form of nicotinamide adenine dinucleotide (NADH) or NADPH. In addition, vitamin $\mathrm{C}$ can reduce the oxidized forms of vitamin E and GSH. There is paucity of information on the role of vitamin $\mathrm{C}$ in $\mathrm{DN}$ despite evidence that it normalizes sorbitol concentration in the blood, scavenges LPO, and regenerates GSH in diabetes [145]. In a prospective cohort study, vitamin $\mathrm{C}$ intake was found to be significantly lower among incident cases of T2DM [155].

\subsection{Vitamin $\mathrm{E}$}

Vitamin E is a group of fat-soluble compounds that includes the AO compound alpha-tocopherol, which is a lipid-soluble AO that increases resistance of LDL-C to oxidation, reduces smooth muscle cell proliferation, and reduces adhesiveness of platelets to collagen [156]. Vitamin E (tocopherols) reacts with hydroxyl radical to form a stabilized phenolic radical which is reduced back to the phenol by ascorbate and NADPH dependent reductase enzymes [154]. It inhibits LPO by scavenging reactive oxygen species and preserving cell membranes. Neurological conditions associated with vitamin E deficiency includes: posterior spinal columns disease, spinocerebellar ataxia, peripheral neuropathy, and optic neuropathy [156].

Vitamin $\mathrm{E}$ has been reported to alleviate symptoms of DM and diabetesinduced complications in animals through reduction in OS biomarkers. In clinical trials, vitamin E did not however show a significant relief of the symptoms of microvascular and macrovascular complications despite reducing OS biomarkers in the subjects [145].

The lack of performance of vitamin E may not however be unconnected to the fact that the design of each study was not targeted directly at diabetes endpoints such as $\mathrm{HbA}_{1 \mathrm{c}}<7 \%$ levels, $\mathrm{BP}<130 / 180 \mathrm{mmHg}$, avoiding hypoglycemic events, and maintaining weights but rather at complications that may have multiple causal factors [145]. Vitamin E supplementation reduced blood glucose and $\mathrm{HbA}_{1 \mathrm{c}}$ levels significantly and had a neuroprotective effect on the total myenteric population, without affecting intestinal area or thickness of the intestinal wall or muscular tunic [145]. 
Vitamin $\mathrm{E}$ is a fat-soluble vitamin which may interact with lipid hydroperoxides and scavenge them. It also participates, together with vitamin C, in GSH regeneration by interaction with LA. Since OS is present during the progression of diabetes and its complications, amelioration of oxidative status, mainly by increasing AO non-enzymatic defenses, has been largely proposed and studied. Several clinical observational trials have particularly studied the correlation between vitamin $\mathrm{E}$ status in plasma and/or diet, and markers of oxidation, inflammation, T2DM incidence, and diabetic complications [163].

Although inverse association has been found for vitamin $\mathrm{E}$ in some studies [164], the association found in other study disappeared after adjustment for cardiovascular risk factors such as obesity, smoking, and hypertension, or have observed no beneficial effect at all [163]. Such contrasting results have also been reported for studies looking association of vitamin $\mathrm{A}$ and $\mathrm{C}$ consumption and amelioration of diabetes status and/or complications [163].

In three prospective observational studies, serum $\alpha$-tocopherol levels were associated with lower risk of type T1DM or T2DM. In another prospective study cohort of more than 4.000 non-diabetic subjects over 23 years, vitamin E intake was significantly associated with a reduced risk of T2DM [155].

On the other hand, in interventional trials with vitamin supplementation, the effects of vitamins E, C and A, alone or in diverse combinations, have yielded barely any promising result. There appears to be no beneficial effect of vitamin supplementation on diabetes or micro-, macrovascular complications [164]. Some of these studies have even evidenced associations between vitamin supplementation and an increased incidence of stroke [164]. Likewise, supplementation with AO vitamins can even block beneficial ROS production during exercise, inhibiting the health-promoting effects of exercise in humans [163].

Information about the antioxidants and drugs with antioxidant properties in the management of diabetes and its complications are given in Tables 20-21. [165]

Table 20 Antioxidants in the management of diabetes and its complications

\begin{tabular}{|l|l|l|}
\hline \multicolumn{1}{|c|}{ Vitamin } & \multicolumn{1}{|c|}{ Target } & \multicolumn{1}{c|}{ Efficacy } \\
\hline Vitamin C & $\begin{array}{l}\text { Patients with type 2 diabetes } \\
\text { mellitus }\end{array}$ & $\begin{array}{l}\text { Decreases fasting plasma } \\
\text { insulin level, decreases } \\
\text { HbA1c level, improves } \\
\text { insulin action }\end{array}$ \\
\hline Vitamin E (alpha tocopherol) & STZ-induced diabetic rats & $\begin{array}{l}\text { Reduces LP, reduces the } \\
\text { activity of GSH-Px and GST }\end{array}$ \\
\cline { 2 - 3 } & $\begin{array}{l}\text { Patients with type 2 diabetes } \\
\text { mellitus }\end{array}$ & $\begin{array}{l}\text { Reduces oxidative stress } \\
\text { indicators, reduce protein } \\
\text { glycosylation, reduces } \\
\text { insulin resistance }\end{array}$ \\
\hline Vanadium & $\begin{array}{l}\text { Patients with type 1 diabetes } \\
\text { mellitus }\end{array}$ & $\begin{array}{l}\text { Scavengers of free radical, } \\
\text { reduces LP in pancreas }\end{array}$ \\
\hline
\end{tabular}




\begin{tabular}{|l|l|l|}
\hline Zinc & STZ-induced diabetic rats & $\begin{array}{l}\text { Induces synthesis of } \\
\text { metallothionein, reduces } \\
\text { retinal LP }\end{array}$ \\
\hline Selenium & $\begin{array}{l}\text { Alloxane-induced diabetic } \\
\text { rats }\end{array}$ & $\begin{array}{l}\text { Increases GSH in liver and } \\
\text { brain }\end{array}$ \\
\hline Beta carotene & $\begin{array}{l}\text { Alloxane-induced diabetic } \\
\text { rats }\end{array}$ & Reduces oxidative LDL \\
\cline { 2 - 3 } & $\begin{array}{l}\text { Patients with type 2 diabetes } \\
\text { mellitus }\end{array}$ & \\
\hline
\end{tabular}

STZ: Streptozocin, GSH-Px: Glutathione peroxidase, GST: Glutathione S-transferase, GSH: Glutathione, LDL: low density lipoprotein, LP: Lipid Peroxidation, Hb: Hemoglobin

Paradoxically, in spite of the solid evidence of increased OS in DM, and the well established actions of vitamins as AOs, the association studies between AO vitamin status and its beneficial effects in DM has no consistent results at all. What is more, interventional studies have failed in demonstrating a favorable effect of vitamin supplementation, discouraging its use as AO therapy for DM [163]. Several reasons have been suggested for these contradictory observations. First, as vitamins may be easily oxidized, a vitamin may have AO or oxidant properties, depending on the presence of other vitamins and the oxidative state in the cells i.e., if the oxidized form of a vitamin is not correctly reversed into the reduced form. Additionally, some vitamins may also activate OS pathways and further increase the OS, such as the activation of protein kinase $\mathrm{C}$ (PKC) by retinoids [163].

Vitamin doses may also be part of the problem, as the effect of vitamins depends on dietary concentrations and/or supplement intake.

Table 21 Drugs with antioxidant properties in diabetes mellitus [165]

\begin{tabular}{|l|l|l|}
\hline Drugs & Mechanism of action & Benefits in diabetes \\
\hline ACEI and ARB & $\begin{array}{l}\text { Inhibition of angiotensin II } \\
\text { and expression of NAD(P)H } \\
\text { oxidase }\end{array}$ & Nephropathy \\
\hline Melatonin & Free radicals scavenger & Nephropathy, neuropathy \\
\hline$\alpha$ - lipoic acid & $\begin{array}{l}\text { Free radicals scavenger, } \\
\text { metal ion chelation, } \\
\text { antioxidant recycling }\end{array}$ & Neuropathy, retinopathy \\
\hline Glibenclamide & Free radical scavenger & \\
\hline Allupurinol & Inhibit xanthine oxidase & \\
\hline
\end{tabular}




\begin{tabular}{|l|l|l|}
\hline Metformin & $\begin{array}{l}\downarrow \text { xanthine oxidase activity, } \\
\text { metal ion chelation, free } \\
\text { radicals scavenger, inhibit } \\
\text { xanthine oxidase and } \\
\text { phosphodiesterase, } \downarrow \text { TNF- } \alpha \\
\text { production, inhibit AGEs }\end{array}$ & Cardiovascular disease \\
\hline Repaglinide & $\uparrow$ GR and GSH level & \\
\hline Phosphodiesterase inhibitor & $\begin{array}{l}\text { Inhibit phosphodiesterase, } \uparrow \\
\text { cAMP \& cGMP }\end{array}$ & \\
\hline Caffeic acid phenethyl ester & Free radicals scavenger & Cardiovascular disease \\
\hline Carvedilol & $\begin{array}{l}\text { Inhibit electrone adduction, } \\
\text { ihibit LP in myocardium } \\
\text { cells, inhibit O2 release } \\
\text { thorugh neutrophil, } \\
\text { improve the body's natural } \\
\text { antioxidant system, free } \\
\text { radical scavengers }\end{array}$ & Cardiovascular disease \\
\hline
\end{tabular}

ACEI: angiotensin converting enzyme inhibitor, ARB: angiotensin receptor blocker, NADPH: Nicotinamide adenine dinucleotide phosphate-oxidase, GSH: gluthathione, GR: glutathione reductase, TNF: tumor necrosis factor, LP: lipid peroxidation, AGE: advanced glycation end product.

The wide variety of doses reached with diet and supplements, and the lack of an established "pharmacological" dose of vitamins, makes it difficult to ascertain the true net effect of vitamin status or supplementation needed to generate beneficial effects. As well, the required dose for $\mathrm{AO}$ effects versus the required for the vitamin's role in the body may differ, which, together with vitamin's bioavailability and its interaction with other vitamins, are caveats for assessing and finding vitamins' effects, if any [163-165].

Other AOs are taurine, acetyl L-carnitine, and $\mathrm{N}$-acetylcysteine which have been demonstrated to reduce the progression of DPN [15].

9.10.2. Strategies targeted against individual oxidative stress pathways [145]

The pathways of hyperglycemia-induced OS discussed earlier are potential therapeutic targets in DPN. Some of the interventions have resulted in specific therapies, for example, aldose reductase inhibitors (ARIs), PKC inhibitors, and anti-AGE agents.

\subsubsection{Aldose reductase inhibitors}

Therefore, ARIs are agents that reduce the flux of glucose into the polyol pathway thereby preventing the harmful effects of excess sorbitol and fructose in neurons. Results from in vivo and in vitro animal studies highlighted the positive effect of inhibiting ARI on DPN [145]. These studies have been the foundation for embarking on several clinical trials with ARIs with AO activities such as Fidarestat (SNK-860), Epalrestat, and Ranirestat (AS-3201) [145]. Among the ARIs 
that have made it to clinical trials, Epalrestat was licensed in Japan while others (e.g., Tolrestat (AY-2773), Zenarestat (FK-366; FR-74366), and Ponalrestat) were withdrawn due to inefficacy or safety concerns [145]. ARIs prevent the progression of DPN, enhance sural motor and sensory nerve conduction velocities (NCV), and improve wrist and ankle F-wave latency together with alleviating neuropathic pain $[145,166]$. In addition, it is reported that the use of $50 \mathrm{mg} 3$ times/d. Eparestat may improve motor and sensory nerve conduction velocity and subjective neuropathy symptoms [145].

\subsubsection{Protein kinase $\mathrm{C}$ inhibitors}

$\mathrm{PKC}$ is involved in the activation of key regulatory proteins responsible for nerve function and synthesis of neurotransmitters. Inhibiting PKC was reported to suppress neuropathic pain. Ruboxistaurin, a specific inhibitor of neuronal protein kinase $\mathrm{C}(\mathrm{PKC1B})$ that possesses antioxidant effects, improves $\mathrm{NCV}$ and endoneurial blood flow in diabetic rats. In clinical trials, Ruboxistaurin reduces the progression of DPN [145] but fails to achieve its primary end-points, vibration detection threshold (VDT) and symptoms reduction. Ruboxistaurin had effects on diabetic DPN in some studies, but the evidence is not enough for meta-analysis and firm conclusion.

\subsubsection{Anti-advanced glucose end-products agents}

Anti-AGE agents prevent the formation and accumulation of AGEs. They also counteract the AGE-receptor for AGE (RAGE) interactions that might aggravate the OS damage in DPN. Examples are benfotiamine, aminoguanidine, and aspirin which are known for their $\mathrm{AO}$ properties through the inhibition of AGEs formation $[7,145]$.

\subsubsection{Benfotiamine}

Benfotiamine (BFT) has been reported to increase transketolase enzyme activity which directs AGE substrates to the pentose phosphate pathway resulting in the reduction of hyperglycemic damage. It also inhibits the increase in UDP-Nacetylglucosamine that induces the hexosamine pathway activity ultimately reducing tissue AGEs [145, 167-169]. In combination with pyridoxamine and cyanocobalamin, BFT improves the vibration perception threshold, motor function, and symptom score $[145,170]$.

It is clear that the correction of thiamine deficiency (TD) must be performed using exogenous vitamin $\mathrm{B}_{1}$, or BFT (monophosphate S-benzoyl-thiamine, highbioavailable liposoluble vitamin $B_{1}$ derivatives). Results of experimental and clinical studies suggest a positive effect of BFT prescription on prevention of diabetic vascular disease progression. BFT broad therapeutic potential has a good efficiency on medications containing soluble thiamine derivatives for the purpose of regulating the activity of free radical processes; correction of endothelial dysfunction in case of CVD, stabilization of clinical and AO effects [145, 170].

BFT can promote neuronal and vascular deficiency correction through participation of NO processes, which have a significant therapeutic potential for the treatment of CVD. The use of thiamine and ALA combination has a great significance in the treatment of diabetic angio-, neuropathy. In particular, it demonstrated that prescription of BFT and ALA to patients with T1DM was followed 
by normalization of hyperglycemia and for $4 \mathrm{wk}$ it promoted the normalization of prostacyclin synthase suppressed by diabetes; increase of transketolase activity in monocytes in 2-3 times [167].

Dysautonomia is a broad term that describes any disease or malfunction of the autonomic nervous system (ANS). Evidence has been produced to indicate that various forms of mild to moderate vitamin deficiencies result in functional changes in the ANS. It is hypothesized that the predictable loss of efficiency in oxidative metabolism is the key to understanding the association of dysautonomia with many different diseases. Mild to moderate hypoxia and/or TD both give rise to exaggeration of centrally controlled mechanisms involved in all survival reflexes, mediated normally through a balanced reaction of the ANS and endocrine system. Together with dietary excesses, particularly in the universal ingestion of sugar, appears to be responsible for initiating long term disease related to the synthesis and use of cellular energy. Failure of ANS cholinergic neurotransmission might follow from TD and/or other cofactors involved in glucose metabolism, exposing the organism to adrenal medullary release of epinephrine [171, 172].

Chronic exposure to moderate and severe hypoxia increases the activity of the ANS and adrenal medulla and TD induces an early functionally significant central muscarinic cholinergic lesion in rat studies [173, 174]. Thus, we aimed to evaluate the effects of BFT (a lipid-soluble thiamine derivative with higher bioavailability than thiamine) on some metabolic and functional parameters in T2DM patients with functional stage of CAN.

To explore the effectiveness of some abovementioned compounds we examined 32 patients with T2DM and advanced stage of CAN, patients were aged between 50-59 years with disease duration 1-6 years and median glycated hemoglobin A1c (HbAlc) $7.1 \% \pm 0.4 \%$. CAN was diagnosed according to previously proposed criteria [5]. The work was done according to the principles of the Helsinki Declaration II and was approved by the Medical Ethics Committee of Danylo Halytsky Lviv National Medical University. All participants signed an informed consent prior to their inclusion in the study. Patients with T2DM and advanced stage of CAN were allocated to two treatment groups: first group received traditional antihyperglycaemic therapy $(\mathrm{n}=15$, control group); patients in group $2(\mathrm{n}=17)$ received in addition to standard treatment-benfotiamine $300 \mathrm{mg} / \mathrm{d}$ [Benfogamma ("WÖRWAG Pharma" GmbH \& Co.KG, Germany)]. The duration of the treatment was three months.

The concentration of glucose in the blood was determined by the glucose oxidase method while $\mathrm{HbA}$ lc level was assessed by using a highly sensitive method of ionexchange liquid chromatography with D-10 analyzer and BIO-RAD reagents (United States). Determination of immunoreactive insulin (IRI) was performed using commercial kits from immunotech insulin immunoradiometric assay reagents (Czech Republic); leptin level-from Immunotech Leptin (Czech Republic) test kits; tumour necrosis factor-alpha (TNF-alpha), interleukin (IL)-6, IL-8 and IL-10-from VectorBest (Russia); high-sensitivity C-reactive protein (hsCRP)-from diagnosis-related group (United States); N-terminal fragment of the prohormone brain natriuretic peptide (NT-proBNP)-from Biomedica (Austria) kits and an enzyme-linked immunosorbent assay analysis technique; thromboxane B2 (TxB2) and 6-ketoprostaglandin F1 alpha (6-keto-PGF1 alpha)-Enzo ${ }^{\circledR}$ Life Sciences (USA). TNF-alpha/ 
IL-10 and TxB2/6-keto-PGF1 alpha ratios were calculated. Lipid metabolism was assessed by the concentration of total cholesterol (TC), triglyceride (TG), lowdensity lipoprotein (LDL)-, high-density lipoprotein (HDL)-, very low-density lipoprotein (VLDL)-cholesterol measurements. The lipid fractions were determined by using HUMAN reagents (Germany) for the analyzer HUMANLAYZER 2000. Homeostasis model assessment (HOMA) insulin resistance index (HOMA-IR) was calculated according to the formula: fasting IRI $(\mathrm{mcIU} / \mathrm{mL}) \mathrm{x}$ fasting glucose $(\mathrm{mmol} / \mathrm{L}) / 22.5$ [175].

Resting 12-lead surface electrocardiography (ECG) with a paper speed of 25 $\mathrm{mm} / \mathrm{s}$ and a signal size of $10 \mathrm{~mm} / \mathrm{mV}$ was recorded in the morning period. The QTc was calculated by dividing the QT interval by the square root of the preceeding $\mathrm{NN}$ interval (Bazett's formula: QTc $=\mathrm{QT} / \sqrt{\mathrm{NN}}$ ) [176]. QT dispersion $(\mathrm{QTd})$ was calculated as the difference between the maximum and minimum QTc. ECG-derived measure of the difference in mean vectors of depolarization and repolarization (QRS-T angle). The absolute difference between the frontal QRS wave axis and T-wave axis was defined as frontal planar QRS-T angle. If such a difference exceeded $180^{\circ}$, the difference was calculated by subtracting from $180^{\circ}$ [177]. We performed resting ECG analysis included measurement of following parameters: heart rhythm, heart rate, conduction intervals and Holter-ECG [(ECG "EC-3H" ("Labtech," Hungary)] analysis included measurement of 24 hours ECG, circadian indexes and following HRV parameters: standard deviation of all NN intervals (SDNN), standard deviation of the means of all $\mathrm{NN}$ intervals for all 5-minute segments of the entire recording (SDANNi), the square root of the mean of the sum of the squares of differences between adjacent NN intervals (RMSSD), the square root of the mean of the sum of the squares of differences between adjacent $\mathrm{NN}$ intervals (pNN50,\%), the HF, LF, VLF, LF/HF.

In order to evaluate the artery stiffness parameters during active and passive periods of the day we have used the device TensioMedTM Arteriograph [monitor blood pressure "ABPM-04» ("Meditech," Hungary) and measured: aorta augmentation index (AIxao), brachial augmentation index (AIxbr), pulse wave velocity (PWV) and ambulatory arterial stiffness index. The program orders the values of the AIxbr and PWV into ranges as follows: optimal values: AIxbr $>-30 \%$, PWV $<7 \mathrm{~m} / \mathrm{s}$; normal values: $-30 \%<$ AIxbr $<-10 \%, 7 \mathrm{~m} / \mathrm{s}<\mathrm{PWV}<10 \mathrm{~m} / \mathrm{s}$; elevated values: $-10 \%<$ AIxbr $<10.0 \%, 10.0 \mathrm{~m} / \mathrm{s}<\mathrm{PWV}<12 \mathrm{~m} / \mathrm{s}$; pathological values: AIxbr $>10 \%, \mathrm{PWV}>12 \mathrm{~m} / \mathrm{s}$ [178].

Statistical analysis was based on the variational method using statistical parametric t-test, nonparametric Wilcoxon t-test and Fisher's Pearson correlation coefficient. Data are presented as mean \pm standard error of the mean (SEM). All tests were performed using the ANOVA (MicroCal Origin v. 8,0) software. Statistical significance was set at $\mathrm{p}<0.05$.

Changes of the adipo-, cyto- and chemokines parameters among patients with T2DM and advanced stage of CAN after 3-mo of BFT therapy are given in Table 22. 
Table 22 Changes of the hsCRP, TNF-alpha, leptin and interleukins in patients with type 2 diabetes mellitus and advanced stage of cardiovascular autonomic neuropathy after 3-mo of therapy

\begin{tabular}{|c|c|c|c|c|}
\hline \multirow[t]{2}{*}{ Parameter } & \multicolumn{4}{|c|}{ Patients with T2DM and advanced stage of CAN $(n=32)$} \\
\hline & Groups & Baseline & After treatment & $\begin{array}{l}\text { \% change from } \\
\text { baseline }\end{array}$ \\
\hline \multirow[t]{2}{*}{ hsCRP (mg/l) } & Control group & $2.87 \pm 0.49$ & $2.68 \pm 0.46$ & $-7.2 \% \pm 1.63 \%$ \\
\hline & Benfotiamine & $2.93 \pm 0.16$ & $2.5 \pm 0.1 \mathrm{a}$ & $-13.6 \% \pm 1.9 \%$ \\
\hline \multirow{2}{*}{$\begin{array}{l}\text { TNF-alpha (pg/ } \\
\text { ml) }\end{array}$} & Control group & $5.81 \pm 0.43$ & $5.49 \pm 0.43$ & $-6.1 \% \pm 1.0 \%$ \\
\hline & Benfotiamine & $5.39 \pm 0.24$ & $4.76 \pm 0.14 \mathrm{a}$ & $-10.2 \% \pm 1.5 \%$ \\
\hline \multirow[t]{2}{*}{ Leptin $(\mathrm{mcg} / \mathrm{l})$} & Control group & $21.05 \pm 1.68$ & $19.51 \pm 1.57$ & $-7.1 \% \pm 1.8 \%$ \\
\hline & Benfotiamine & $20.9 \pm 1.87$ & $19.6 \pm 1.83$ & $-6.4 \% \pm 1.3 \%$ \\
\hline \multirow[t]{2}{*}{ IL-6 (pg/ml) } & Control group & $5.53 \pm 0.53$ & $5.11 \pm 0.42$ & $-5.8 \% \pm 1.77 \%$ \\
\hline & Benfotiamine & $5.71 \pm 0.3$ & $4.78 \pm 0.2 \mathrm{a}$ & $-15.4 \% \pm 2.04 \%$ \\
\hline \multirow[t]{2}{*}{ IL-8 (pg/ml) } & Control group & $7.25 \pm 0.49$ & $6.93 \pm 0.46$ & $-3.9 \% \pm 1.57 \%$ \\
\hline & Benfotiamine & $7.13 \pm 0.49$ & $6.63 \pm 0.41$ & $-6.3 \% \pm 2.01 \%$ \\
\hline \multirow[t]{2}{*}{$\mathrm{IL}-10(\mathrm{pg} / \mathrm{ml})$} & Control group & $16.72 \pm 2.54$ & $15.65 \pm 2.26$ & $-3.7 \% \pm 2.34 \%$ \\
\hline & Benfotiamine & $16.15 \pm 1.88$ & $14.79 \pm 1.48$ & $-6.19 \% \pm 2.71 \%$ \\
\hline \multirow{2}{*}{$\begin{array}{l}\text { TNF- } \alpha / \text { IL-10 } \\
(\%)\end{array}$} & Control group & $42.5 \pm 5.47$ & $41.4 \pm 4.79$ & $-0.5 \% \pm 2.3 \%$ \\
\hline & Benfotiamine & $38.6 \pm 4.28$ & $35.9 \pm 3.3$ & $-4.32 \% \pm 2.76 \%$ \\
\hline
\end{tabular}

The results are presented as absolute values and as \% change from baseline, $(\triangle \%$, Mean \pm SEM $) ;{ }^{a} p<0.05,{ }^{b} p<0.01$, compared to baseline. T2DM: type 2 diabetes mellitus; CAN: cardiovascular autonomic neuropathy; hsCRP: high reactive C-reactive protein; TNF-alpha: tumor necrosis factor-alpha; IL: interleukin.

We found out that the HbA1c of patients with T2DM and advanced stage of CAN was not statistically significant influenced by the treatment $(p>0.05)$. Benfotiamine prescription to patients with T2DM and advanced stage of CAN did not cause any significant changes in TC, TG, LDL-C, HDL-C, VLDL-C concentrations and leptin levels $(\mathrm{p}>0.05)$, while it probably helped reduce the IRI concentration $[-12.7 \% \pm 1.4 \%,(\mathrm{p}<0.05)]$.

The use of BFT in the comprehensive treatment of T2DM helped reducing hsCRP $[-15.4 \% \pm 2.04 \%,(\mathrm{p}<0.05)]$, TNF-alpha $[-10.2 \% \pm 1.6 \%,(\mathrm{p}<0.05)]$ and IL-6 concentrations $[-13.3 \% \pm 2.1 \%,(\mathrm{p}<0.05)]$. BFT prescription to patients 
with T2DM and advanced stage of CAN did not cause any significant changes in IL-8, IL-10 concentrations and TNF-alpha/IL-10 ratio.

Treatment with BFT among patients with T2DM and advanced stage of CAN led to a significant decrease of the TxB2, TxB2/6-keto-PGF1 alpha levels. The use of BFT in the treatment of advanced stage of CAN did not cause any significant changes in NT-proBNP and 6-keto-PGF1 alpha levels $(\mathrm{p}>0.05)$. Changes of the NT-proBNP, TxB2, 6-keto-PGF1 alpha levels and TxB2/6-keto-PGF1 alpha ratio parameters among patients with T2DM and advanced stage of CAN after 3-mo of BFT prescription are given in Table 23.

Table 23. Changes of the NT-proBNP, TxB2, 6-keto-PG1F1 alpha levels and TxB2/6-keto-PGF1 alpha ratio in patients with type 2 diabetes mellitus and advanced stage of cardiovascular autonomic neuropathy after 3-mo of therapy

\begin{tabular}{|l|l|c|c|c|}
\hline \multirow{2}{*}{ Parameter } & \multicolumn{4}{|c|}{ Patients with T2DM and advanced stage of CAN $(\mathrm{n}=32)$} \\
\cline { 2 - 5 } & \multicolumn{1}{|c|}{ Groups } & Baseline & After treatment & $\begin{array}{c}\text { \% change from } \\
\text { baseline }\end{array}$ \\
\hline $\begin{array}{l}\text { NT-proBNP } \\
(\mathrm{fmol} / \mathrm{ml})\end{array}$ & Control group & $408.67 \pm 28.85$ & $\mathbf{3 9 6 . 8 7} \pm \mathbf{2 8 . 8 4}$ & $-3.0 \% \pm 1.1 \%$ \\
\cline { 2 - 5 } & Benfotiamine & $423.2 \pm 37.19$ & $398.3 \pm 31.66 \mathrm{a}$ & $-5.2 \% \pm 1.73 \%$ \\
\hline TxB2 (ng/ml) & Control group & $194.8 \pm 9.29$ & $197.5 \pm 10.09$ & $+1.68 \% \pm 2.37 \%$ \\
\cline { 2 - 5 } & Benfotiamine & $201.1 \pm 12.93$ & $\mathbf{1 6 7 . 7} \pm \mathbf{8 . 6} \mathbf{a}$ & $-15.75 \% \pm 2.45 \%$ \\
\hline $\begin{array}{l}\text { 6-keto-PGF1 } \\
\text { alpha (ng/ml) }\end{array}$ & Control group & $85.1 \pm 4.32$ & $84.0 \pm 3.86$ & $-0.79 \% \pm 1.78 \%$ \\
\cline { 2 - 5 } & Benfotiamine & $97.6 \pm 5.89$ & $105 \pm 9.0$ & $+1.68 \% \pm 0.12 \%$ \\
\hline $\begin{array}{l}\text { TxB2/6- } \\
\text { ketoPGF1 } \\
\text { alpha (\%) }\end{array}$ & Control group & $2.35 \pm 0.14$ & $2.41 \pm 0.16$ & $+2.48 \% \pm 1.71 \%$ \\
\cline { 2 - 5 } & Benfotiamine & $2.09 \pm 0.12$ & $\mathbf{1 . 6 8} \pm \mathbf{0 . 1 2} \mathbf{~ a}$ & $-19.7 \% \pm 3.99 \%$ \\
\hline
\end{tabular}

The results are presented as absolute values and as \% change from baseline, $(\Delta \%$, Mean $\pm \mathrm{SEM}) ;{ }^{\mathrm{a}} \mathrm{p}<0.05$, compared to baseline. T2DM: Type 2 diabetes mellitus; CAN: Cardiovascular autonomic neuropathy; NT-proBNP: N-terminal prohormone of brain natriuretic peptide; TxB2: Thromboxane B2; 6-ketoPGF1 alpha: 6-ketoprostaglandinF1 alpha.

Obtained results of this study could prove that prescription of BFT is accompanied by more significant decrease of QTc, QTd and QRS-T angle parameters compared to patients in control group. The features of the QTc, QTd and QRS-T angle parameters in patients with T2DM and advanced stage of CAN after treatment with BFT are given in Table 24. 
Table 24 Changes of the QTc, QTd and QRS-T parameters in patients with type 2 diabetes mellitus and advanced stage of cardiovascular autonomic neuropathy after 3-mo of therapy

\begin{tabular}{|l|l|c|c|c|}
\hline \multirow{2}{*}{ Parameter } & \multicolumn{4}{l}{ Patients with T2DM and advanced stage of CAN (n=32) } \\
\cline { 2 - 5 } & \multicolumn{1}{|c|}{ Groups } & Baseline & After treatment & $\begin{array}{c}\text { \% change from } \\
\text { baseline }\end{array}$ \\
\hline \multirow{2}{*}{ QTc $(\mathrm{ms})$} & Control group & $433.4 \pm 6.45$ & $427.8 \pm 4.72$ & $-1.1 \% \pm 1.44 \%$ \\
\cline { 2 - 5 } & Benfotiamine & $423.1 \pm 5.76$ & $\mathbf{3 9 2 . 4} \pm \mathbf{7 . 7 4 a}$ & $-7.3 \% \pm 1.36 \%$ \\
\hline \multirow{2}{*}{ QTd $(\mathrm{ms})$} & Control group & $50.3 \pm 4.53$ & $46.0 \pm 4.98$ & $-5.6 \% \pm 6.97 \%$ \\
\cline { 2 - 5 } & Benfotiamine & $58.1 \pm 3.94$ & $\mathbf{3 9 . 4} \pm \mathbf{4 . 4 2 a}$ & $-27.7 \% \pm 9.0 \%$ \\
\hline \multirow{2}{*}{ QRS-T angle $\left(^{\circ}\right)$} & Control group & $78.0 \pm 6.44$ & $69.7 \pm 4.27$ & $-6.1 \% \pm 5.52 \%$ \\
\cline { 2 - 5 } & Benfotiamine & $88.6 \pm 6.4$ & $\mathbf{5 9 . 3} \pm \mathbf{5 . 1 5 a}$ & $-24.4 \% \pm 10.2 \%$ \\
\hline
\end{tabular}

The results are presented as absolute values and as \% change from baseline, $(\Delta \%$, Mean $\pm \mathrm{SEM}) ;{ }^{\mathrm{a}} \mathrm{p}<0.01$, compared to baseline. T2DM: type 2 diabetes mellitus; CAN: cardiac autonomic neuropathy; QTc: corrected QT interval; QTd: QT interval dispersion; QRS-T angle: spatial QRS-T angle.

Table 25 Changes of the time-domain heart rate variability parameters in patients with type 2 diabetes mellitus and advanced stage of cardiovascular autonomic neuropathy after 3-mo of therapy

\begin{tabular}{|l|l|c|c|c|}
\hline \multirow{2}{*}{ Parameter } & \multicolumn{4}{|l|}{ Patients with T2DM and advanced stage of CAN (n=32) } \\
\cline { 2 - 5 } & \multicolumn{1}{|c|}{ Groups } & Baseline & After treatment & $\begin{array}{c}\text { \% change from } \\
\text { baseline }\end{array}$ \\
\hline \multirow{2}{*}{ SDNN (ms) } & Control group & $94.7 \pm 4.84$ & $91.3 \pm 3.33$ & $-0.82 \% \pm 2.73 \%$ \\
\cline { 2 - 5 } & Benfotiamine & $98.8 \pm 2.73$ & $103.8 \pm 2.7$ & $+5.3 \% \pm 1.64 \%$ \\
\hline \multirow{2}{*}{ SDANNi (ms) } & Control group & $72.3 \pm 4.08$ & $74.7 \pm 3.33$ & $+4.72 \% \pm 2.67 \%$ \\
\cline { 2 - 5 } & Benfotiamine & $81.8 \pm 3.18$ & $87.4 \pm 2.78$ & $+7.7 \% \pm 2.33 \%$ \\
\hline \multirow{2}{*}{ RMSSD (ms) } & Control group & $19.1 \pm 1.36$ & $18.7 \pm 0.77$ & $+0.96 \% \pm 3.67 \%$ \\
\cline { 2 - 5 } & Benfotiamine & $21.3 \pm 1.42$ & $23.1 \pm 1.35$ & $+9.48 \% \pm 2.37 \%$ \\
\hline \multirow{2}{*}{ pNN50 (\%) } & Control group & $3.9 \pm 0.42$ & $3.7 \pm 0.23$ & $+7.1 \% \pm 9.59 \%$ \\
\cline { 2 - 5 } & Benfotiamine & $4.94 \pm 0.55$ & $\mathbf{7 . 4 1} \pm \mathbf{1 . 0 6 a}$ & $+45.9 \% \pm 7.91 \%$ \\
\hline
\end{tabular}

The results are presented as absolute values and as \% change from baseline, $(\Delta \%$, Mean $\pm \mathrm{SEM}) ;{ }^{\mathrm{a}} \mathrm{p}<0.05$, compared to baseline. T2DM: type 2 diabetes mellitus; CAN: cardiac autonomic neuropathy; SDNN: standard deviation of all NN intervals; SDANNi: standard deviation of the means of all NN intervals for all 5-minute segments of the entire recording; RMSSD: the square root of the mean of the sum of the squares of differences between adjacent NN intervals; 
pNN50 - percentage of differences greater than 50 ms between adjacent sinus NN intervals.

Treatment with BFT among patients with T2DM and advanced stage of CAN led to a significant decrease of the pNN50 and did not affect the SDNN, SDANNi and RMSSD parameters. The features of the time-domain HRV parameters in patients with T2DM and advanced stage of CAN after BFT administration are given in Table 25.

Table 26 Changes of the spectral heart rate variability parameters during the active period in patients with type 2 diabetes mellitus and advanced stage of cardiovascular autonomic neuropathy after 3-mo of therapy

\begin{tabular}{|l|l|c|c|c|}
\hline \multirow{2}{*}{ Parameter } & \multicolumn{4}{|l|}{ Patients with T2DM and advanced stage of CAN $(\mathrm{n}=32)$} \\
\cline { 2 - 5 } & \multicolumn{1}{|c|}{ Groups } & Baseline & After treatment & $\begin{array}{c}\text { \% change from } \\
\text { baseline }\end{array}$ \\
\hline \multirow{2}{*}{ VLF $\left(\mathrm{ms}^{2}\right)$} & Control group & $1113.6 \pm 98.1$ & $1103.9 \pm 72.8$ & $+1.81 \% \pm 2.63 \%$ \\
\cline { 2 - 5 } & Benfotiamine & $1128.4 \pm 80.34$ & $1241.2 \pm 75.1$ & $+11,5 \% \pm 1,99 \%$ \\
\hline \multirow{2}{*}{ LF $\left(\mathrm{ms}^{2}\right)$} & Control group & $390.8 \pm 21.98$ & $388.5 \pm 14.74$ & $+1.6 \% \pm 3.72 \%$ \\
\cline { 2 - 5 } & Benfotiamine & $414.8 \pm 21.0$ & $474.9 \pm 21.9$ & $+16.8 \% \pm 4.98 \%$ \\
\hline \multirow{2}{*}{ HF $\left(\mathrm{ms}^{2}\right)$} & Control group & $242.1 \pm 18.5$ & $244.5 \pm 13.6$ & $+4.1 \% \pm 4.15 \%$ \\
\cline { 2 - 5 } & Benfotiamine & $267.7 \pm 17.61$ & $\mathbf{3 2 6 . 4} \pm \mathbf{1 6 . 9 6 a}$ & $+25.8 \% \pm 5.58 \%$ \\
\hline \multirow{2}{*}{ LF/HF } & Control group & $1.66 \pm 0.06$ & $1.63 \pm 0.06$ & $-1.6 \% \pm 3.07 \%$ \\
\cline { 2 - 5 } & Benfotiamine & $1.58 \pm 0.04$ & $1.47 \pm 0.05$ & $-6.3 \% \pm 2.9 \%$ \\
\hline
\end{tabular}

The results are presented as absolute values and as \% change from baseline, $(\Delta \%$, Mean $\pm \mathrm{SEM}) ;{ }^{\mathrm{a}} \mathrm{p}<0.05$, compared to baseline. T2DM: type 2 diabetes mellitus; CAN: cardiac autonomic neuropathy; VLF: very low frequency power, total spectral power of all $\mathrm{NN}$ intervals between 0.003 and $0.04 \mathrm{~Hz}$; LF: low frequency power, total spectral power of all $\mathrm{NN}$ intervals between 0.04 and 0.15 $\mathrm{Hz}$; HF: high frequency power, total spectral power of all $\mathrm{NN}$ intervals between 0.15 and $0.4 \mathrm{~Hz}$; LF/HF: sympathetic/parasympathetic ratio.

Obtained results of this study could prove that treatment with BFT is accompanied by significant increase of HF parameters during the active period compared to patients in control group. Changes of the spectral HRV parameters during the active and the passive period in patients with T2DM and advanced stage of CAN after 3-mo of BFT therapy are given in Tables 26-27. 
Table 27 Changes of the spectral heart rate variability parameters during the passive period in patients with type 2 diabetes mellitus and advanced stage of cardiovascular autonomic neuropathy after 3-mo of therapy

\begin{tabular}{|l|l|c|c|c|}
\hline \multirow{2}{*}{ Parameter } & \multicolumn{4}{|l|}{ Patients with T2DM and advanced stage of CAN $(\mathrm{n}=32)$} \\
\cline { 2 - 5 } & \multicolumn{1}{|c|}{ Groups } & Baseline & After treatment & $\begin{array}{c}\text { \% change from } \\
\text { baseline }\end{array}$ \\
\hline \multirow{2}{*}{ VLF $\left(\mathrm{ms}^{2}\right)$} & Control group & $1441.1 \pm 106.7$ & $1439.3 \pm 84.7$ & $+1.83 \% \pm 2.47 \%$ \\
\cline { 2 - 5 } & Benfotiamine & $1440.7 \pm 90.9$ & $1572.4 \pm 85.1$ & $+10.3 \% \pm 1.98 \%$ \\
\hline \multirow{2}{*}{ LF $\left(\mathrm{ms}^{2}\right)$} & Control group & $509.1 \pm 17.82$ & $502.1 \pm 13.0$ & $-0.6 \% \pm 2.55 \%$ \\
\cline { 2 - 5 } & Benfotiamine & $524.1 \pm 21.4$ & $588.5 \pm 25.8$ & $+12.7 \% \pm 3.31 \%$ \\
\hline \multirow{2}{*}{ HF $\left(\mathrm{ms}^{2}\right)$} & Control group & $340.5 \pm 25.3$ & $326.7 \pm 19.01$ & $-1.9 \% \pm 3.38 \%$ \\
\cline { 2 - 5 } & Benfotiamine & $332.5 \pm 18.39$ & $\mathbf{3 9 6 . 6} \pm \mathbf{1 9 . 0 a}$ & $+21.1 \% \pm 4.17 \%$ \\
\hline \multirow{2}{*}{ LF/HF } & Control group & $1.57 \pm 0.08$ & $1.59 \pm 0.07$ & $+1.6 \% \pm 1.92 \%$ \\
\cline { 2 - 5 } & Benfotiamine & $1.61 \pm 0.05$ & $1.5 \pm 0.04$ & $-6.1 \% \pm 2.75 \%$ \\
\hline
\end{tabular}

The results are presented as absolute values and as \% change from baseline, $(\Delta \%$, Mean $\pm \mathrm{SEM}) ;{ }^{\mathrm{a}} \mathrm{p}<0.05$, compared to baseline. T2DM: type 2 diabetes mellitus; CAN: cardiac autonomic neuropathy; VLF: very low frequency power, total spectral power of all NN intervals between 0.003 and $0.04 \mathrm{~Hz}$; LF: low frequency power, total spectral power of all $\mathrm{NN}$ intervals between 0.04 and 0.15 $\mathrm{Hz}$; HF: high frequency power, total spectral power of all $\mathrm{NN}$ intervals between 0.15 and $0.4 \mathrm{~Hz}$; LF/HF: sympathetic/parasympathetic ratio.

Treatment with BFT is accompanied by significant increase of HF parameters during the passive period compared to patients in control group. Changes of the spectral HRV parameters during the passive period in patients with T2DM and advanced stage of CAN after 3-mo of BFT are given in Table 27.

Treatment with BFT among patients with T2DM and advanced stage of CAN led to a significant decrease of PWV parameters during the passive period compared to patients in control group. Changes of the arterial stiffness parameters during the passive period in patients with T2DM and advanced stage of CAN after 3-mo of BFT are given in Table 28. 
Table 28 Changes of the arterial stiffness parameters during the passive period in patients with type 2 diabetes mellitus and advanced stage of cardiovascular autonomic neuropathy after 3-mo of therapy

\begin{tabular}{|l|l|c|c|c|}
\hline \multirow{2}{*}{ Parameter } & \multicolumn{4}{|l|}{ Patients with T2DM and advanced stage of CAN (n=32) } \\
\cline { 2 - 5 } & \multicolumn{1}{|c|}{ Groups } & Baseline & After treatment & $\begin{array}{c}\text { \% change from } \\
\text { baseline }\end{array}$ \\
\hline \multirow{2}{*}{ AIxao (\%) } & Control group & $33.3 \pm 1.95$ & $30.1 \pm 1.39$ & $-6.1 \pm 4.14 \%$ \\
\cline { 2 - 5 } & Benfotiamine & $32.5 \pm 1.48$ & $28.8 \pm 1.47$ & $-11.4 \pm 2.02$ \\
\hline \multirow{2}{*}{ AIxbr (\%) } & Control group & $-4.5 \pm 3.15$ & $-6.5 \pm 2.71$ & $-17.7 \pm 17.5 \%$ \\
\cline { 2 - 5 } & Benfotiamine & $-6.7 \pm 2.21$ & $-10.7 \pm 2.23$ & $-42.9 \pm 8.65 \%$ \\
\hline \multirow{2}{*}{ PWV (m/s) } & Control group & $10.5 \pm 0.42$ & $10.1 \pm 0.41$ & $-3.63 \pm 1.47 \%$ \\
\cline { 2 - 5 } & Benfotiamine & $11.1 \pm 0.28$ & $\mathbf{1 0 . 0 5} \pm \mathbf{0 , 3 a}$ & $-9.4 \pm 1.22 \%$ \\
\hline
\end{tabular}

The results are presented as absolute values and as \% change from baseline, $(\Delta \%$, Mean $\pm \mathrm{SEM}) ;{ }^{\mathrm{a}} \mathrm{p}<0.05$, compared to baseline. T2DM: type 2 diabetes mellitus; CAN: cardiac autonomic neuropathy; AIxao: aorta augmentation index; AIxbr: brachial augmentation index; PWV: pulse wave velocity.

As a result of our studies, it was found out that treatment with BFT contributed to a decrease in resting tachycardia $[110$ to 96 beats/min $(p<0.05)]$, improvement of subjective feeling and increase in tolerance of exercise loading. In addition in the majority of the patients with diabetic polyneuropathies (DPN) we observed the decrease and/or disappearance of pain, paresthesia, frequency of muscle cramps, improvement and/or restoration of tactile, vibration and temperature sensitivity.

Diabetes might be considered as TD state, if not in absolute terms at least relative to the increased requirements deriving from accelerated and amplified glucose metabolism in non-insulin dependent tissues that, like the vessel wall, are prone to complications [162]. Low levels of thiamine and increased renal clearance have been found in both T1DM and T2DM [179, 180]. Some oxidized thiamine metabolites (thiochrome and oxydihydrothiochrome) could also play role in diabetes complications' pathogenesis. It is reported that plasma thiamine levels are decreased by $75 \%$ in T2DM patients and are associated with increased renal clearance and fractional excretion of thiamine [181]. The conventional indicator of thiamine sufficiency, erythrocyte transketolase (TKT) activity, is masked in clinical diabetes by increased protein levels of thiamine transporter-1 (THTR-1) and thiamin transporter-2 (THTR-2). The deficiency of thiamine in clinical diabetes may increase the fragility of vascular cells to the adverse effects of hyperglycaemia and there by the increase of the risk of developing microvascular complications. A suppression of TKT activity, and subsequent down-regulation of the hexose monophosphate (HMP) shunt, resulting in accumulation of glyceraldehyde 3-phosphate (GA3P), fructose 6-phosphate (F6-P), and dihydroxyacetone phosphate (DHAP) may be at least one mechanism in the development of diabetes-induced vascular damage and other comorbidities [182-185]. 
It is hypothesized that genetic variability in TKT might contribute to susceptibility to early DPN $[186,187]$. TKT is indeed pertinent to the pathogenesis of diabetic microangiopathic complications. When activated in the cell by thiamine, TKT determines the diversion of F6-P and GA3P to the pentose-phosphates pathway (PPP), in this way it exercises a protective effect against three pathogenetic mechanisms of microvascular complications, i.e. the glucose-driven hexosamine, PKC, and advanced glycation end products (AGE's) pathways [188, 187]. The connection between genetic variability of TKT and nerve function measures in newly diagnosed diabetic patients were demonstrated, which can favour the identification of patients with a deficiency in TKT defensive mechanisms (and as such suitable candidates for therapeutic intervention) $[188,187]$. Therefore, changes in thiamine levels may be masked by an increase in THTR expression [189]. Thiamine levels and thiamine-dependent enzyme activities have been reduced in DM. Genetic studies provide opportunity to link the relationship between thiamine and DM (such as TKT, the high affinity thiamine transporter (SLC19A2) gene, the transcription factor Specificity Protein 1 (Sp1), $\alpha$-1-antitrypsin, and tumor protein p53) [189]. Experimental diabetes was also associated with tissue specific TD characterised by a marked decrease of plasma thiamine concentration and decreased activity of the thiamine-dependent enzyme of TKT and decreased levels of TKT protein in renal glomeruli [189].

Thiamine and its derivatives have been demonstrated to prevent the activation of the biochemical pathways [increased flux through the polyol pathway, formation of AGE's, activation of PKC, and increased flux through the hexosamine biosynthesis pathway (HBP) induced by hyperglycaemia in DM. Thiamine definitively plays a role in the diabetic endothelial vascular diseases (micro and macroangiopathy), lipid profile, retinopathy, nephropathy, cardiopathy, and neuropathy [190, 189].

Thiamine acts as a coenzyme for TKT and for the pyruvate dehydrogenase and $\alpha$-ketoglutarate dehydrogenase complexes, enzymes which play a fundamental role intracellular glucose metabolism. TKT and glucose-6-phosphate dehydrogenase (G6PDH), the rate-limiting enzymes of the PPP, are inhibited in the diabetic heart under basal conditions [191]. There is sufficient evidence to indicate that THTR's and TKT activity are suppressed in diabetes. Suppressed TKT activity can lead to accumulation of GA3P, F6-P, and DHAP. Because these are also intermediates of early stage glycolysis, their accumulation is exacerbated by hyperglycaemia-induced mitochondrial free radical production, which inhibits the glycolytic enzyme glyceraldehydes-3-phosphate dehydrogenase (GAPDH). Restoring TKT activity via BFT or thiamin supplementation can increase the flux of glucose into HMP shunt, and also increase flux of GA3P, F6P, and DHAP into HMP shunt and away from hyperglycaemia-induced pathways that lead to vascular damage [184].

Inhibition of AGE's proved to be more effective with thiamine pyrophosphate and pirydoxamin than aminoguanidine. The lipid-soluble derivate BFT is characterised by five times higher bioavailability compared to the water-soluble compound and was shown to be effective in the treatment of DPN [192-194]. Experiences from cardiology indicate that long-term increases in HRV and reduction in sudden cardiac death have only been shown with lipophilic agents that readily penetrate the blood nerve/blood brain barrier. In accordance with these observations 
experimental data indicate a preventive effect of BFT on the development of CAN [195].

Thiamine supplementation can prevent hyperglycaemia-driven reductions in cell replication and proliferation as well as decreasing AGE's formation and reducing lactate levels. In vitro studies with BFT and thiamine have shown a reduction in PKC activation in the glomeruli and decreased glomerular AGE's levels. BFT has been shown to prevent increased markers of HBP activity, intracellular AGE's formation, intracellular PKC activity and nuclear factor kappa-light-chain-enhancer of activated $\mathrm{B}$ cells $(\mathrm{NF}-\mathrm{kB})$ activation seen with in vitro hyperglycaemic damage [196]. High-dose therapy of thiamine and BFT suppressed AGE's accumulation in the peripheral nerve and reversed diabetic neuropathy potentially by reducing the levels of triose phosphates via activation of TKT [196].

Oral BFT in combination with the antioxidant alpha-LA acid treatment normalizes production of angiopoietin-2, a marker of increased intracellular methylglyoxals in endothelial cells which contribute to AGE's formation, and $\mathrm{N}$-acetylglucose modified protein, a marker of HBP activity. Both thiamine and BFT have been shown to reduce AGE's formation in experimental diabetes. Treatment with both thiamine and BFT has been shown to reduce activation of the polyol pathway of glucose metabolism and to increase TKT expression in the presence of hyperglycaemia [197]. Activation of AGE receptors in DM, found on glomerular endothelial cells, cardiomyocytes, pericytes, and podocytes, stimulates postreceptor signaling, intracellular reactive oxygen species formation, and altered gene expression, leading to vascular damage [197].

Atherogenic DLP was associated with an increased risk of silent MI and silent CHD in patients with T2DM and LDL-C levels $<3.35 \mathrm{mmol} / \mathrm{L}$. Specific management of atherogenic DLP might help reducing the high residual burden of CHD [198]. Cardiovascular disease in T2DM is linked to increased risk of atherosclerosis, increased levels of TG-rich lipoproteins and enhances hepatic lipogenesis. High dose of thiamine therapy $(70 \mathrm{mg} / \mathrm{kg})$ prevented increased in plasma TC and TG in diabetes-induced rats but it did not reverse decrease of HDL-C. However, a lower dose of thiamine $(7 \mathrm{mg} / \mathrm{kg})$ and the BFT were ineffective in preventing these lipid profiles [199]. As a result of our previous studies, it has been established that the combined treatment of patients with T2DM and advances stage of CAN with omega-3 PUFAs, BFT, and $\alpha$-LA resulted in significant positive changes in TC, TG, LDL-C and HDL-C [200]. The results obtained, in our opinion, open up prospects for research in this direction.

Benfotiamine significantly reduced inflammatory $(10-300 \mathrm{mg} / \mathrm{kg})$ and neuropathic $(75-300 \mathrm{mg} / \mathrm{kg})$ nociception in non-diabetic and diabetic rats [193]. The anti-inflammatory effects of BFT were investigated in lipopolysaccharide (LPS)stimulated murine microglial cell line BV-2. It has been that BFT remodels activated microglia to acquire the shape that is characteristic of non-stimulated BV-2 cells. In addition, BFT significantly decreased production of pro-inflammatory mediators such as inducible form of nitric oxide synthase (iNOS) and nitric oxide (NO); cyclooxygenase-2 (COX-2), heat-shock protein 70 (Hsp70), TNF-alpha, IL-6, whereas it increased anti-inflammatory IL-10 production in LPS-stimulated BV-2 microglia. Moreover, BFT suppressed the phosphorylation of extracellular signalregulated kinases 1/2 (ERK1/2), c-Jun N-terminal kinases (JNK) and a serine/ 
threonine protein kinase $(\mathrm{Akt} / \mathrm{PKB})$. Treatment with specific inhibitors revealed that BFT-mediated suppression of NO production was via JNK1/2 and Akt pathway, while the cytokine suppression includes ERK1/2, JNK1/2 and Akt pathways. Finally, the potentially protective effect is mediated by the suppression of translocation of NF- $\mathrm{kB}$ in the nucleus [201]. In murine macrophages, BFT also blocked the expression of COX-2 and its product, prostaglandin E2, by LPS-induces cytotoxicity. In addition, BFT significantly prevented LPS-induced macrophage death and monocyte adhesion to endothelial cells [202]. These anti-inflammatory effects of BFT are mediated through the regulation of the arachidonic acid pathway in macrophages [203]. These findings suggest that thiamine may play a role in modulating the inflammatory process. Therefore, BFT may have therapeutic potential for neurological diseases by inhibiting inflammatory mediators and enhancing antiinflammatory factor production.

The results obtained in this study indicate that BFT contributed to a decrease in the level of hsCRP, TNF-alpha and IL-6 (Table 22). Obtained results could witness, that prescription of BFT may lead to decrease of the proinflammatory immune response. Previously, we showed that BFT administration in patients with T1DM with DPN (150 mg in dragee tid for $2 \mathrm{mo}$ ) promotes to a significant increase of superoxide dismutase, gluthation peroxidase activities, reduced gluthatione (GSH) content; decrease of malondialdegide concentration in the RBCs', conjugated dienes, adenosine diphosphate-induced platelet aggregation [204]. The protective effect of high-dose thiamine on detrusor contractility and on progression of diabetic cystopathy in streptozotocin (STZ)-diabetic rats was some of the findings directed to the effect of thiamine on diabetic autonomic neuropathy. The explanations about positive thiamine effects were confined to hydrophobic thiamine metabolites that fulfill an important function under oxidative stress (OS) and nitrosyl stress. Thiamine protects nervous tissue probably by inhibiting NOdependent tyrosine nitration and subsequent formation of dityrosine and interprotein tyrosine-tyrosine crosslink [205, 206]. The identification of the association of polymorphisms related to the genes of thiamine and TKT with DPN might be a first step in defining a DPN genetic risk profile with potential therapeutic repercussions. There is moderate evidence from preclinical experimental models that high-dose thiamine and BFT (1) inhibit the HMP, AGE's formation, and diacylglycerol-PKC through the TKT activation; (2) target at various surrogate markers of hyperglycaemia-induced pathological processes and (3) can delay the progression of microangiopathic complications [187].

Clinical trials in both diabetic nephropathy and DPN have shown some effect on microalbuminuria and symptomatic DPN, although with some controversy due to their limitations such as low number, poor design, short duration, confounding bias of dietary source of thiamine, different phenotypes, and inadequately defined outcomes [187]. Thus, there is still a need for high-quality, well-designed clinical trials of appropriate size and duration to bridge the gap in evidence and to allow final recommendations to be established on the use of thiamine and BFT as a disease-modifying treatment of diabetic complications [187]. Benfotiamine treatment counters diabetes-induced cardiac mechanical dysfunction at the cellular level, associated with reduction in OS but not AGE's formation or cardiac protein carbonyl formation. This apparent discrepancy in BFT-elicited action on AGE's formation 
and OS (the ratio of GSH to oxidized GSH) seems to indicate that other mechanism(s) may predominantly contribute to diabetes-induced OS and cardiac contractile dysfunction in current experimental setting. Possible candidates may include alteration in glucose metabolism and PKC activation), although further study is warranted to verify involvement of these signaling pathways and beneficial effects of BFT against diabetic complications [184]. A decrease in the blood supply to the heart caused by atherosclerosis or thrombosis is known to induce MI [189]. The results of our study revealed that the appointment of BFT to patients with T2DM and advances stage of CAN were accompanied by a decrease of the TxB2 concentration and TxB2/6-keto-PGF1 alpha levels, which may contribute to the improvement of the functional state of the prostacyclin I2-thromboxane A2 system.

Benfotiamine supplementation may provide benefits in the prevention of other diabetes-related vascular and neuronal comorbidities. Benfotiamine supplementation for 14 weeks $(100 \mathrm{mg} / \mathrm{kg} / \mathrm{d})$ in STZ-induced diabetic mice completely corrected hyperglycaemia-induced disruptions in $\mathrm{Ca}^{2+}$ homeostasis and mechanical functioning of cardiomyocytes. Additionally, STZ-induced diabetic rats showed significant increases in expression of BNP, a marker for heart failure, but thiamin repletion almost completely reversed BNP elevations. The results of our study showed no statistically significant effect of BFT on NT-proBNP levels in diabetic patients (Table 23). In the same animal model, thiamin supplementation prevented the formation of cardiac fibrosis observed in STZ-induced diabetic rats that did not receive supplementation [184].

Cardiac OS is involved in heart failure that is induced by thiamine deprivation in rats. These findings suggest that thiamine modulates OS [207, 189]. Nitric oxide synthase (NOS) is an enzyme that is involved in the synthesis of NO, which regulates a variety of important physiological responses, including cell migration, the immune response, and apoptosis. Endothelial NOS (eNOS) and NO may play an important role in attenuating cardiac remodeling and apoptosis. Benfotiamine reduces OS and activates eNOS to enhance the generation and bioavailability of $\mathrm{NO}$, and it subsequently improves the integrity of vascular endothelium to prevent sodium arsenite-induced experimental vascular endothelial dysfunction [208, 189].

In patients with diabetic CAN, QTc prolongation should be avoided due to the risk of inducing severe ventricular arrhythmias. The relationship between diabetic CAN and CHD is intricate, as HRV is decreased in patients with CHD and decreased HRV has been shown to be a powerful predictor of cardiac mortality after MI $[209,187]$. The results of our study showed that the appointment of BFT in the treatment of patients with T2DM and advances stage of CAN for 3 mo contributed to a decrease in the QTc, QTd and QRS-T angle parameters.

CAN may contribute to an increase in artery rigidity, a cardiovascular risk factor [209, 187]. As a result of our studies, it was found that the use of BFT in the treatment of patients with T2DM and advances stage of CAN for 3 mo is accompanied by a statistically significant decrease in the PWV during the passive period of the day.

In conclusion, the positive influences of BFT on decrease of the IRI, hsCRP, TNF-alpha, IL-6, TxB2 concentration, TxB2/6-keto-PGF1 alpha levels; QTc, QTd, QRS-T angle, PWV during the passive period of the day, increase of pNN50; HF parameters during the active and passive periods of the day by us are partly 
confirmed by its neurotropic, cardioprotective, angioprotective and cytoprotective properties; suggests the feasibility of its usage in the complex treatment of patients with T2DM and advances stage of CAN. Proposed scheme of the influence of BFT on metabolic and functional disorders in patients with T2DM and CAN is presented in Figure 1.

The mechanism of BFT influence on diabetic CAN pathogenesis is not wellknown. Thus, further investigations aimed to understand the mechanism of action and confirmation of the beneficial effect of BFT on biochemical parameters, dynamics of independent cardiovascular tests, daily monitoring of electrocardiography, arterial wall stiffness parameters among patients with T2DM and advances stage of CAN and its associated comorbidities may be needed to validate this clinical findings.

It was found out that BFT contributed to decrease of the IRI, hs-CRP, TNF- $\alpha$, IL-6, TxB2 concentration, TxB $/ 6$-keto-PGF $\alpha$ levels; QTc, QTd, QRS-T angle; to increase of high-frequency component of HRV during the active and passive periods; pulse wave velocityparameters during the passive period of the day. Therefore, the positive influences of BFT is partly confirmed by its neurotropic, cardioprotective, angioprotective and cytoprotective properties; suggests the feasibility of its usage in the complex treatment of patients with T2DM and advances stage of CAN [89].

\subsubsection{Aminoguanidine}

Aminoguanidine has been reported to react with 3-deoxyglucosone, a precursor of AGE, thereby trapping the reactive carbonyls and preventing the formation of AGEs although it has been withdrawn from clinical trial as a result of toxicity $[145,210]$. Aminoguanidine improves capacity of nerve velocity, increases blood flow, inhibits the formation of AGEs, delays the emergence and development of albuminuria. Analysis of controlled trials confirmed quite aminoguanidine high efficiency among patients with DPN, but the development of a number of side effects terminated their application. The use of aminoguanidine derivatives is accompanied by clinical efficacy and lack of adverse side effects $[10,35]$. The results are promising, but need further clinical double-blind, placebo-controlled studies.

\subsubsection{Aspirin}

Aspirin has been reported to inhibit the production of pentosidine, a crosslinking AGE, by scavenging free radicals and chelating metal ions in collagen incubated with glucose in vitro [211]. 


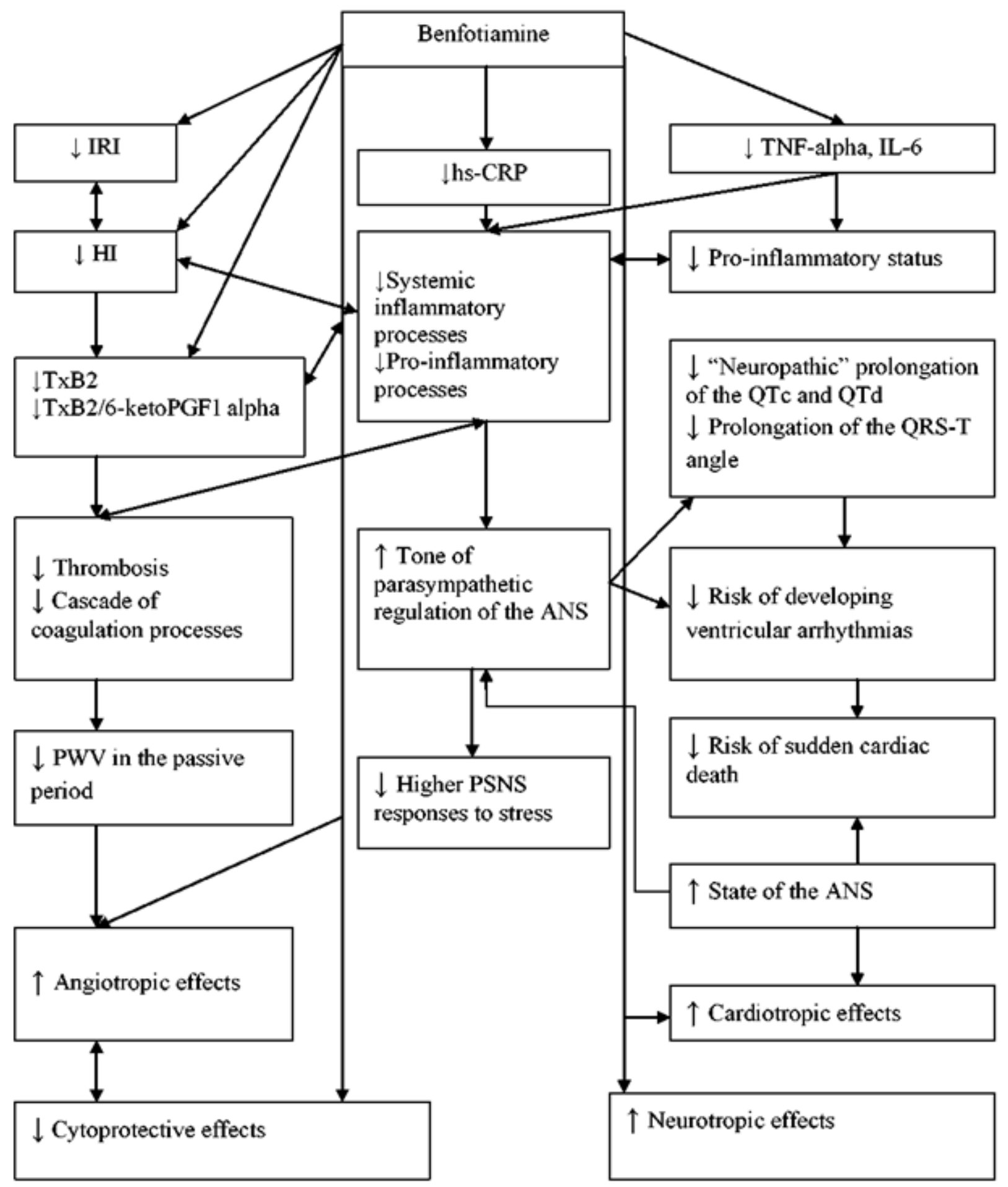

Figure 1 Proposal scheme of the influence of benfotiamine on metabolic and functional disorders in patients with type 2 diabetes mellitus and cardiovascular autonomic neuropathy.

6-keto-PFG1 alpha: 6-keto-prostoglandin F1 alpha; ANS: autonomic nervous system; HI: hyperinsulinemia; hsCRP: high-sensitivity C-reactive protein; IL-6: interleukin-6; IRI: immunoreactive insulin; QRS-T angle: electrocardiogram (ECG)derived measure of the difference in mean vectors of depolarization and repolarization; TNF-alpha: tumour necrosis factor-alpha; TxB2/6-keto-PGF1 alpha: ratio thromboxane $\mathrm{B} 2 / 6-$ ketogrostaglandin $\mathrm{F} 1$ alpha; TxB2: thromboxane $\mathrm{B} 2$. 


\subsubsection{Strategies targeted at mitochondria [145]}

It has been demonstrated that excess superoxide anion radicals, hydroxyl radicals, and $\mathrm{H}_{2} \mathrm{O}_{2}$ are produced during the generation of adenosine triphosphate (ATP) in mitochondria under hyperglycemic conditions contributing to increased oxidative damage [145].

\subsubsection{Coenzyme Q}

Coenzyme Q (a mitochondrial antioxidant) or ubiquinone may decrease OS not only by quenching reactive oxidant species but also by "recoupling" mitochondrial oxidative phosphorylation, thereby reducing superoxide production $[155,158] . \mathrm{CoQ}_{10}$ supplements can be either the oxidized form (ubiquinone) or reduced form (ubiquinol) as both forms seem pretty equally potent in increasing circulating levels of total $\mathrm{CoQ}_{10}$ in the body. "Total $\mathrm{CoQ}_{10}$ " refers to the sum of both forms, since $\mathrm{CoQ}_{10}$ can readily swap between forms as it acts in the body [212]. Ubiquinone and ubiquinol form a pair of molecules known as a REDOX couplet (reduction/ oxidation) which is a property that is crucial for the functioning of $\mathrm{CoQ}_{10}$ within the electron transport chain, where it transports electrons from complex I and II to complex III. $\mathrm{CoQ}_{10}$ is an important micronutrient acting on the electron transport chain of the mitochondria with two major functions:

(1) synthesis of ATP;

(2) a potent antioxidant.

Deficiency in $\mathrm{CoQ}_{10}$ is often seen in patients with $\mathrm{T} 2 \mathrm{DM} \cdot \mathrm{CoQ}_{10}$ also has the ability to prevent LPO from either inhibiting lipid peroxyl radicals and has been noted to restore $\alpha$-tocopherol from its radical state back to its AO state [212]. Protein carbonylation has also been noted to be reduced with $\mathrm{CoQ}_{10}$ (direct inhibition of protein oxidation)but has been noted to not influence the conversion of NO into peroxynitrate. Via its $\mathrm{AO}$ potential, ubiquinone can protect DNA from excess oxidation from $\mathrm{H}_{2} \mathrm{O}_{2}$ and potentially act as an anticarcinogen (as noted in human lymphocytes at least) [212].

Deficiency in $\mathrm{CoQ}_{10}$ is often present among patients with $\mathrm{T} 2 \mathrm{DM}$ due to various reasons. As a potent antioxidant, $\mathrm{CoQ}_{10}$ is assumed to scavenge excessive ROS and provide protection to cells, especially mitochondria from oxidative damage. Therefore, restoration of $\mathrm{CoQ}_{10}$ level among patients with $\mathrm{T} 2 \mathrm{DM}$ by supplementation of exogenous $\mathrm{CoQ}_{10}$ could potentially alleviate $\mathrm{OS}$, preserve mitochondrial function, and eventually lead to improvement of glycemic control. In $\mathrm{DM}, \mathrm{CoQ}_{10}$ has been reported to show promising therapeutic potential. However, low bioavailability of these AOs in mitochondria in vivo has been a problem. To overcome this challenge, $\mathrm{AO}$ agents have been developed to target the mitochondria by conjugation to lipophilic cations exploiting the negative membrane potential of the organelle. The standard dose for $\mathrm{CoQ}_{10}$ is generally $90 \mathrm{mg}$ for a low dose and $200 \mathrm{mg}$ for the higher dose, taken once daily with a meal due to its reliance on food for absorption. Dose-dependence is not commonly observed with $\mathrm{CoQ}_{10}$ supplementation and 90 $\mathrm{mg}$ tends to be the best cost-effective dose [212].

In addition to the antioxidants mentioned, a number of commonly used drugs have been reported to have $\mathrm{AO}$ activity, in addition to their primary pharmacological property. For example, gemfibrozil, a lipid-lowering fibrate, was previously reported to have AO actions. Anti- hyperlipidemic statins are thought to exert AO effects. 
In addition, it has been demonstrated that at least a part of the beneficial vascular effects of thiazolidinediones (TZDs) are linked with their AO properties [155].

\subsubsection{Telmisartan}

Telmisartan is a well-known unique angiotensin II (Ang II) type 1 receptor blocker (ARB) that exerts a powerful AO effect. A recent clinical study has shown it to be more effective in renoprotection as compared to other ARB class of drugs like losartan and valsartan owing to the difference in their intrinsic pharmacokinetic and physiochemical properties. Furthermore, a number of properties like the best binding affinity to Ang II type 1 receptors, the maximum plasma half life and the highest lipophilicity among the presently available ARBs make this molecule a long lasting antioxidant [213]. It basically functions by enhancing the activity of superoxide scavenging enzyme i.e., SOD, by down regulating NADPH oxidase (Nox), an enzyme responsible for superoxide production. It has also been shown to reduce albuminuria and decrease diabetic nephropathy conversion from the early to the evident form. Ang II directs the synthesis of superoxide through the activation of Nox. Therefore, blockage of Ang II pathway through Ang II type 1 receptors by telmisartan ameliorates OS. Telmisartan has a potential neuro-protective effect on PNP; this is mediated through its anti-inflammatory effects and its dual properties as an ARB, and a partial PPAR- $\gamma$ igand [213].

Usual adult dose for hypertension. Initial dose: $40 \mathrm{mg}$ orally once a day. Maintenance dose: 40 to $80 \mathrm{mg}$ orally once a day. Usual adult dose for cardiovascular risk reduction: $80 \mathrm{mg}$ orally once a day.

\subsubsection{Metformin}

Both American and European guidelines recommend metformin as the firstline agent for the pharmacological management of T2DM and preventing its complications [3]. It possesses AO property and causes reduction of albumin excretion rate in the urine of diabetic patients. In addition, it decreases the production of AGEs, improves free radical defense system by its ability to directly scavenge oxygenated free radicals and thereby reduces intracellular ROS levels. By reducing the generation of ROS, metformin shows renoprotective effect in diabetic patients which has been confirmed by an increment in the ratio of ATP/adenosine monophosphate (ATP/AMP) in the renal tissue of normoglycemic rats treated with drug after $8 \mathrm{wk}$ as compared to streptozotocin-induced diabetic rats which shows decrease in the level of both ATP and AMP. Recent research also indicates that metformin intake can ameliorate the increased MDA level and can restore the biochemical alteration and modulation of both enzymatic and non-enzymatic antioxidants thereby indicating the clinical use of this antidiabetic drug in the treatment of. The glycemic control-independent neuroprotective and antineuropathic effects of metformin recently reported in animal studies [214].

Usual adult dose for T2DM. Initial dose: $500 \mathrm{mg}$ PO bid or $850 \mathrm{mg}$ PO qd. Dose titration: increase in $500 \mathrm{mg}$ weekly increments or $850 \mathrm{mg}$ every 2 weeks as tolerated. Maintenance dose: $2.000 \mathrm{mg}$ daily in divided doses. Maximum dose: $2.500 \mathrm{mg} / \mathrm{d}$. 


\subsubsection{Pioglitazone}

Thiazolidinedione drugs such as pioglitazone are approved by the FDA for the treatment of T2DM. TZDs also reduce the molecular and behavioral sequelae of neurological disease. Positive and protective effects of TZD group of drugs, like pioglitazone, in the amelioration of AO enzyme levels in renal histopathology and renal tissue associated with diabetic nephropathy has recently been investigated by many researchers. Treatment of rats with pioglitazone normalizes bowman capsular volume, drops down the amount of endothelial constitutive eNOS in the glomerular vascular endothelium, and improves the glomerular hyperfiltration. Increased expression of nuclear transcription factor p65 (NF- $\kappa B$ p65) in renal tubules and glomeruli during diabetic nephropathy has been reduced by pioglitazone therapy thereby showing protection from renal pathophysiology. But TZDs has limited clinical uses due to the occurrence of fluid retention, hemodilution, and heart failure in about $15 \%$ of patients.

Usual adult dose for T2DM. Initial dose: $15-30 \mathrm{mg}$ PO with meal qDay initial; may increase dose by $15 \mathrm{mg}$ with careful monitoring to $45 \mathrm{mg}$ qDay maximum.

Some drugs with AO properties which have antioxidant effect in patients with $\mathrm{DM}$ are shown in Table 20 [165].

\subsubsection{Triple antioxidant therapy}

Participants with T1DM with early complications were randomly assigned to a combination AO regimen or to placebo. Allopurinol (300 mg qd), ALA (600 $\mathrm{mg}$ bid) and nicotinamide (750 $\mathrm{mg}$ bid), or matched PO placebos were administered for 24 mo. The administration of each individual active drug or placebo component was titrated in consecutive weeks (first ALA, then nicotinamide, finally allopurinol) such that the participant began receiving full therapeutic doses of all the medications 3 wk postrandomisation. In cohort of T1DM patients with mild-to-moderate CAN, a combination $\mathrm{AO}$ treatment regimen did not prevent progression of CAN, had no beneficial effects on myocardial perfusion or DPN, and may have been detrimental. However, a larger study is necessary to assess the underlying causes of these findings [84].

\subsection{Correction of vascular endothelial dysfunction}

Optimization of myocardial energy metabolism is based on increased myocardial glucose oxidation, which enhances cardiac function and protects myocardial fibers from ischemic and reperfusion injuries. Myocardial use of glucose in case of chronic disease may be improved due to intake of the medicines, that can improve fatty acids metabolism and inhibit their oxidation $[215,216]$.

\subsubsection{Trimetazidine}

New therapeutic approach has been implemented after advent of trimetazidinethe first representative of a new class of metabolic agents- inhibitors of 3-ketoacyl coenzyme A thiolase. Trimetazidine reduces oxidation of fatty acids; stimulates glucose intake; restores the link between glycolysis and carbohydrate oxidation, which leads to the formation of ATP, reducing $\mathrm{O}_{2}$ consumption; redirects fatty acids towards phospholipids; increases cell tolerance to ischemic and reperfusion 
injuries; increases the oxidation of glucose, the activity of $\mathrm{Na}^{+}, \mathrm{K}^{+}$-ATPase and $\mathrm{Ca}^{2+}$-pomps in the sarcoplasmic reticulum. Anti-ischemic properties of trimetazidine do not depend on changes in hemodynamics and are associated with a distinct recovery of mechanical function after ischemia, which makes it recognized as cardyo-cytoprotective agent. Trimetazidine prescription improves glucose metabolism; reduces endothelin-1 among patients with diabetic cardiomyopathy, that is taken to have effect on the vascular endothelium; accompanied by a significant positive changes in ejection fraction (EF) parameters among patients with HF; improves quality of life parameters and NYHA functional class [215, 216].

\subsubsection{Perhexiline}

Another pharmacological agent that facilitates the inhibition of metabolism of fatty acids is perhexiline. Perhexiline prescription to patients with heart failure significantly contributes to the improvement of $\mathrm{EF}, \mathrm{VO}_{2} \max$ and quality of life. Unfortunately, the clinical use of this medicament is limited because of the risk of hepatotoxicity and PNP [216, 217].

\subsubsection{Ranolazine}

Ranolazine is the third antianginal pharmacological agent with a potential of metabolism modificator. However, the following factors do not allow to implement its use: the degree of inhibition of fatty acids metabolism is limited by physiological indicators; ranolazine prescription associates with the possibility of QTc interval prolongation [216,218].

\subsection{Beta blockers}

Beta blockers show a protective effect on myocard in case of ischemia. Prescription of $\beta$-adrenergic receptor blockers for T2DM with CAD and CAN has significant pathogenetic grounds as high sympathetic activity that is followed by CAN, accelerate the development of CVD and significantly affects prognosis. In addition, several studies demonstrated the ability of $\beta$-blockers to reduce the incidence of "silent" myocardial ischemia episodes and improve prognosis among these patients. However, adrenergic receptors $\beta$-blockers negatively affect the performance of glycemic profile, increase the risk of hypoglycemia, showing a negative effect on blood lipid profile and can provoke acute heart failure [89]. The above described events occur with prescription of non-selective $\beta$-blockers. Selective $\beta$-adrenergic receptor blockers, including metoprolol, are free of side effects, including the effectiveness of metoprolol in the treatment of CVD demonstrated in numerous controlled studies. Metoprolol has cardioprotective properties; improves prognosis among patients with $\mathrm{CAD}$; has a fair tolerance in case of prolonged use. Cardioselective $\beta$-blockers can also balance the effects of autonomic dysfunction in particular by resisting sympathetic stimulation they can restore parasympathetic-sympathetic balance. However, traditional antianginal agents that affect hemodynamic parameters ( $\beta$-blockers, $\mathrm{Ca}^{2+}$ antagonists, etc.), have lower tolerance among elderly due to the high risk of the interaction of pharmacological agents with a significant incidence of side effects [2, 8, 219-221].

Some beta blockers, particularly the $\beta_{1}$-selective beta blockers exert endothelial protective effects. Nebivolol, a $\beta_{1}$-antagonist with $\beta_{2,3}$-agonist property, improves 
endothelium-dependent vasodilator responses in patients with essential hypertension [212-224]. The effect of nebivolol on endothelial function appears to be mediated by increasing NO release and reducing prothrombotic blood levels of fibrinogen, homocysteine and plasminogen activator inhibitor-1 [223, 224]. Carvedilol, a nonselective $\beta_{1^{-}}$and $\beta_{2^{-}}$antagonist with $\alpha$-antagonist property, also improves endothelium-dependent responses in patients with essential hypertension but this seems to be related to its antioxidant capacity $[223,224]$. The combination of carvedilol with an angiotensin-converting enzyme (ACE) inhibitor produces more beneficial effect on endothelial function than each drug alone in hypertensive patients with obesity [225]. Thus, this type of $\beta$-blockers and its combination are suitable for the treatment of endothelial dysfunction associated with hypertension, atherosclerosis, and probably DM [224].

\subsection{Dihydropyridine calcium channel blockers}

Blockers of $\mathrm{Ca}^{2+}$-channels show a protective effect on myocard in case of ischemia. In terms of correction of cell power the most pathogenetically efficient option is the use of $\mathrm{Ca}^{2+}$ blockers, however they only eliminate secondary dysfunction links of oxidative phosphorylation in mitochondria.

Nicardipine and nifedipine protect against ROS-induced endothelial cell death and lose of glutathione in cultured cells [224, 226]. Benidipine exerts an endothelial protective effect against OxLDL induced ROX generation in human endothelial cells $[221,224]$. Israpidine improves endothelial function in cholesterol-fed rabbit $[224,227,228]$. Thus, the endothelial protective effect of dihydropyridine calcium channel blockers is mainly mediated by their antioxidant actions related to reduction in LPO and associated ROS generation [224, 226]. In addition, some dihydropyridines such as, amlodipine, azelnidipine and nifedipine were shown to exert an antiinflammatory action as indicated by decreased C-reactive protein and IL-6 levels as well as leucocyte activation [224, 229]. Amlodipine or in combination with an renin inhibitor improves endothelial dysfunction in hypertensive patients, which seems to be linked to its NO-releasing action and anti-inflammatory effect [224, 230, 231]. In addition, the combination of amlodipine with a statin induces more favorable vascular effects than each drug alone in rats with hypertension or DM $[232,233]$. Thus, in addition to hypertension, dihydropyridines may also be useful for the treatment of endothelial dysfunction.

\subsection{Phosphodiesterase-5 inhibitors}

Phosphodiesterase-5 (PDE5) is highly specific for hydrolysis of cyclic nucleotides monophosphate, such as cyclic guanosine monophosphate (cGMP), which is a molecular messenger involved in regulation of vascular function, axon guidance, the modulation of DPN and pain perception [234-236]. PDE5 inhibitors including sildenafil, tadalafil, and vardenafil, are primarily used as pharmacological agents for the treatment of erectile dysfunction, but they also have a potential therapeutic application for the treatment of neurovascular dysfunction, neuroinflammatory and neurodegenerative diseases by inducing accumulation of cGMP and activation of cGMP dependent protein kinase, e.g., protein kinase G, signaling pathways [236, 237]. Clinical study demonstrates that PDE5 inhibitors are safe and generally well tolerated with no serious side effects in patients. 
Sildenafil improves vascular function and blood supply to the vasa neurvorum while ameliorating neurological function of neuropathy in diabetic patients [238].

The considerably longer duration of action for tadalafil may permit less frequent dosing and could potentially reduce adverse effects associated with treatment. Moreover, the absorption and activity of tadalafil is unaffected by food ingestion, age, diabetes, or mild to moderate hepatic insufficiency. Also, tadalafil did not lower systemic BP in clinical trials. Therefore, we investigated the therapeutic effect of tadalafil for DN [239].

The angiopoietin-Tie signaling system was identified as a vascular-specific receptor tyrosine kinase pathway that is essential for vessel development. PDE5 inhibitor-induced activation of the cGMP/PKG and ANG/Tie2 signaling pathways promotes neurovascular remodeling both directly through these signaling pathways to ameliorate neurovascular function, and indirectly via endothelial cells and Schwann cells, which produce neurotrophic factors and provide a permissive restorative microenvironment in the sciatic nerve. Both direct and indirect approaches, in concert, improve neurological function of diabetic neuropathy [240].

\subsection{Ivabradine, the cardiac pacemaker «funny» (I(f)) inhibitor}

Ivabradine is a heart-rate-lowering agent that acts by selectively and specifically inhibiting the If, a mixed $\mathrm{Na}^{+}-\mathrm{K}^{+}$inward current that controls the spontaneous diastolic depolarization in the sinoatrial node and hence regulates the HR [241]. Ifcurrent is an inward current carried by $\mathrm{Na}+$ and $\mathrm{K}+$, activated by hyperpolarization and conducted by hyperpolarization-activated cyclic nucleotide-gated channels (f-channels) [242]. Ifcurrent participates in the spontaneous depolarization during Phase 4 of the action potential and plays a crucial role in the pacemaker activity of pacemaker cells located in the sinus node and atrioventricular node. Inhibition of this current by ivabradine slows down HR and exerts cardioprotective effects $[224,233,241]$, which may involves pleiotropic actions of ivabradine. The effects of ivabradine on human endothelial dysfunction are controversial. Several studies did not observe significant improvement in flow-mediated vasodilation by ivabradine in patients with stable CHD and in patients with T2DM [221, 242, 243]. In addition, in patients with stable of CHD without heart failure, the additional ivabradine plus standard treatment did not improve outcome but was associated with increased frequency of atrial fibrillation, questioning the utility of this drug in the treatment of stable coronary disease [224, 244-247].

\subsection{Thrombosis prevention and treatment}

Platelets obtained from patients with T2DM and tested in vitro are characterized by a real ability to aggregate under the influence of ADP, adrenaline, collagen, arachidonic acid, and thrombin. Aggregation of platelets is significantly increased in the second, irreversible phase, which depends on the transformation of arachidonic acid into labile prostacyclin and thromboxane. Thus, the possibility of ADP receptors of platelet membranes blocking is a pathogenetically justified measure. Prescription of antiplatelet agents, namely acetylsalicylic acid (ASA), clopidogrel and others can help prevent blood clots, stenocardia and development of MI. The active clopidogrel metabolite irreversibly binds to ADP receptor on the platelet membrane, which leads to inhibition of adenylate cyclase; inhibition of ADP-dependent secretion 
of platelet granules; inhibition of ADP-dependent process of binding fibrinogen receptor to the platelet membrane; does not affect the expression of receptors directly; blocks myointymal proliferation in case of vascular damage; unlike ASA does not affect the activity of cyclooxygenase. Effect of clopidogrel and ASA synergy is demonstrated in the study of plateletex vivo. However, clopidogrel is more effective pharmacological agent within the frames of the combined risk of MI, stroke, and the syndrome of "sudden death" reduction [248, 249].

\subsection{Treatment of orthostatic hypotension}

Treatment for $\mathrm{OH}$ is challenging and usually involves both pharmacological and non pharmacological interventions. Pharmacological therapies must balance an increase in standing BP against prevention of supine hypotension. Orthostatic hypotension is notoriously difficult to manage because the standing BP must be raised without causing hypertension when the patient lies down.

Non-pharmacologic treatment should be the initial approach. Physical activity and exercise should be encouraged to avoid deconditioning, which is known to exacerbate orthostatic intolerance. Volume repletion with fluids and salt is central to the management of $\mathrm{OH}$. To increase venous return, supportive stockings should be worn during the day and removed at bedtime. Patients should be advised to avoid hot baths, to get out of bed or stand up slowly and if their diabetes is being treated with insulin, patients should administer insulin injections while lying down $[8,250,251]$. Low-dose fludrocortisones may be beneficial in supplementing volume repletion in some patients, although there are growing concerns on risk of supine hypertension $[8,250,251]$.

Pharmacologic approaches may also be employed. The mineralicorticoid, fludrocortisone, together with supplementary salt increases plasma volume. Unfortunately, it is generally ineffective until edema develops, which carries a risk of causing congestive HF and hypertension [82]. The mixed adrenergic agonist, ephedrine, the $\alpha-1$ adrenergic agonist, midodrine, and the $\alpha-2$ adrenergic agonist, clonidine have each been found to be effective in some patients, but it is important to begin with a low dose and titrate upward to minimize the various symptoms associated with their use [82]. Finally, the somatostatin analog, octreotide may also help some patients who experience particularly refractory $\mathrm{OH}$ after eating [7].

Orthostatic hypotension can be aggravated by different forms of therapy (e. $g$, tricyclic antidepressant [amitriptyline]) used for the treatment of other complications (e. g., painful sensory neuropathy). Therefore, careful attention to other medications that may aggravate $\mathrm{OH}$ in these patients is mandatory $[8,252]$. Enhancement of ganglionic transmission via the use of pyridostigmine (inhibitor of acetylcholinesterase) improved symptoms and orthostatic BP with only modest effects in supine BP for patients with POTS [8]. Similarly, the use of $\beta$-adrenergic blockers may benefit the tachycardia and anticholinergics, the orthostatic bradycardia. Pyridostigmine has also been shown to improve HRV in healthy young adults [8, 253]. In patients with pooling of blood in the splanchnic bed, somatostatin (Octreotide) may be of value, and in patients with contracted plasma volume, treatment with erythropoietin is recommended [8].

As neurogenic $\mathrm{OH}$ is in large part a consequence of the failure of norepinephrine release from sympathetic neurons, the administration of sympathomimetic medications 
is central to the care of patients whose symptoms are not controlled with other measures [3, 39, 82]. Midodrine, a peripheral, selective, direct $\alpha 1$-adrenoreceptor agonist, is an FDA-approved drug for the treatment of $\mathrm{OH}[3,39,82]$. Midodrine should be titrated gradually to efficacy. It should be used only when patients intend to be upright or seated to minimize supine hypertension. Recently, droxidopa (northera) was approved by the FDA for the treatment of neurogenic $\mathrm{OH}$ but not specifically for patients with $\mathrm{OH}$ due to $\mathrm{DM}[3,39]$. 


\section{CONCLUSION}

CAN is common and often underdiagnosed complication of DM which is strongly associated with increased rate of cardiovascular morbidity and mortality. As the progression of cardiovascular denervation is partly reversible or can be slowed down in the early stages of disease, it is recommended to perform screening for that complication in patients with DM. Assessment of CAN is possible through a variety of methods, but the "gold" standard clinical tests are CARTs. The intensive glycemic control, life style modification and CVD risk factors management is the basic prevention and treatment tools, but the unified algorithm and known disease modifying treatment is lacking. Pathogenetic treatment of CAN includes: balanced diet and physical activity, optimization of glycemic control, treatment of DLP, antioxidants, vitamins, correction of vascular endothelial dysfunction, prevention and treatment of thrombosis, in severe cases treatment of orthostatic hypotension. The revival of interest in vascular hypothesis of CAN, OS index, neurotrophic hypothesis and importance of autoimmune disorders in the development and progression of CAN opens up new areas of treatment. The perspective methods include prescription of medications, that increase blood flow through the vasa vasorum, including prostacyclin analogues, TXA 2 blockers and drugs that contribute into strengthening and/or normalization of $\mathrm{Na}^{+}, \mathrm{K}^{+}$-ATPase (phosphodiesterase-5 inhibitors), ALA, BFT, DGLA, $\omega-3$ PUFAs, and the simultaneous prescription of ALA, $\omega-3$ PUFAs and DGLA. In addition the combined administration of $\alpha$-LA, $\omega-3$ PUFAs and benfotiamine promotes reduction of chronic inflammation markers and increase of heart rate variability parameters, that might be useful in preventing the development and progression of CAN. Development of orthostatic hypotension is associated with severe or advanced CAN and prescription of nonpharmacological and pharmacological, in the foreground midodrine and fludrocortisone acetate, treatment methods are necessary. 


\section{REFERENCES}

1. Ogurtsova K, da Rocha Fernades JD, Huang Y, Linnenkamp U, Guariguata L, Cho NH, Cavan D, Shaw JE, Makaroff LE. IDF Diabetes Atlas: Global estimates for the prevalence of diabetes for 2015 and 2040.

2. Marazzi G, Volterrani M, Rosano GM. Metabolic agents in the management of diabetic coronary patients: a new era. Int J Cardiol 2008; 127: 124-125.

3. American Diabetes Association. Standards of medical care in diabetes-2017. Diabetes Care 2017; 40 Suppl 1: 88-98.

4. Tesfaye S, Boulton AJ, Dyck PJ, Freeman R, Horowitz M, Kempler P, Lauria G, Malik RA, Spallone V, Vinik A, Bernardi L, Valensi P. Diabetic neuropathies: update on definitions, diagnostic criteria, estimation of severity, and treatments. Diabetes Care 2010; 33: 2285-2293.

5. Spallone V, Ziegler D, Freeman R, Bernardi L, Frontoni S, Pop-Busui R, Stevens M, Kempler P, Hilsted J, Tesfaye S, Low P, Valensi P. Cardiovascular autonomic neuropathy in diabetes: clinical impact, assessment, diagnosis, and management. Diabetes Metab Res Rev 2011; 27: 639-653.

6. Dimitropoulos G, Tahrani AA, Stevens MJ. Cardiac autonomic neuropathy in patients with diabetes mellitus. World J Diabetes 2014; 5: 17-39.

7. Edwards JL, Vincent A, Cheng T, Feldman EL. Diabetic neuropathy: Mechanisms to management. Pharmacol Ther 2008; 120: 1-34.

8. Vinik AI, Ziegler D. Diabetic cardiovascular autonomic neuropathy. Circulation 2007; 115: 387-397.

9. Vinik AI, Erbas T. Diabetic autonomic neuropathy. Handb Clin Neurol 2013; 117: 279-294.

10. Callaghan BC, Cheng HT, Stables CL, Smith AL, Feldman EL. Diabetic neuropathy: clinical manifestations and current treatments. Lancet Neurol 2012; 11: 521-534.

11. Cannon CP. Combination therapy in the management of mixed dyslipidaemia. J Intern Med 2008; 263: 353-365.

12. Bril V. Treatments for diabetic neuropathy. J Peripher Nerv Syst 2012; 17 Suppl 2: 22-27.

13. Tandon N, Ali MK, Narayan KM. Pharmacologic prevention of microvascular and macrovascular complications in diabetes mellitus: implications of the results of recent clinical trials in type 2 diabetes. Am J Cardiovasc Drugs 2012; 12: 7-22.

14. Soares-Miranda L, Sandercock G, Vale S, Santos R, Abreu S, Moreira C, Mota J. Metabolic syndrome, physical activity and cardiac autonomic function. Diabetes Metab Res Rev 2012; 28: 363-369.

15. Hosseini A, Abdolla M. Diabetic neuropathy and oxidative stress: Oxid Med Cell Longer 2013; 2013: 168039.

16. Vinik AI, Nevoret ML, Casellini C, Parson H. Diabetic neuropathy. Endocrinol Metab Clin North Am 2013; 42: 747-787.

17. Isik A, Firat D. Bilateral intra-areolar polythelia. Brest 2017; Jun 7: $1-2$.

18. Isik A, Soyturk M, Süleyman S, Firat D, Peker K, Yilmaz I, Celebi F. Correlation of bowel wall thickening seen using computerized tomography with 
colonoscopies: A preliminary study. Surg Laparosc Endosc Percutan Tech 2017; 27: $154-157$.

19. Ziegler D. Cardiovascular autonomic neuropathy: clinical manifestations and measurement. Diabetes Rev 1999; 7: 300-315.

20. Vinik AI, Maser RE, Mitchell BD, Freeman R. Diabetic autonomic neuropathy. Diabetes Care 2003; 26: 1553-1579.

21. Rolim Luiz Clemente de Souza Pereira, Sá João Roberto de, Chacra Antonio Roberto, Dib Sérgio Atala. Diabetic cardiovascular autonomic neuropathy: risk factors, clinical impact and early diagnosis.Arq Bras Cardiol 2008; 90: e24-e32.

22. Valensi P, Pariès J, Attali JR; French Group for Research and Study of Diabetic Neuropathy. Cardiac autonomic neuropathy in diabetic patients: influence of diabetes duration, obesity, and microangiopathic complications-the French multicenter study. Metabolism 2003; 52: 815-820.

23. Gaede P, Vedel P, Parving HH, Pedersen O. Intensified multifactorial intervention in patients with type 2 diabetes mellitus and microalbuminuria: the Steno type 2 randomised study. Lancet 1999; 353: 617-622.

24. Gaede P, Vedel P, Larsen N, Jensen GVH, Parving HH, Pedersen O. Multifactorial intervention and cardiovascular disease in patients with type 2 diabetes. N Engl J Med 2003; 348: 383-393.

25. The Diabetes Control and Complications Trial Research Group. The effect of intensive diabetes therapy on measures of autonomic nervous system function in the Diabetes Control and Complications Trial (DCCT). Diabetologia 1998; 41: 416-423.

26. Gaede P, Lund-Andersen H, Parving HH, Pedersen O. Effect of a multifactorial intervention on mortality in type 2 diabetes. N Engl J Med 2008; 358: $580-591$.

27. Kempler P, Tesfaye S, Chaturvedi N, Stevens LK, Webb DJ, Eaton S, Kerenyi Z, Tamas G, Ward JD, Fuller JH; EURODIAB IDDM Complication Study Group. Autonomic neuropathy is associated with increased cardiovascular risk factors: the EURODIAB IDDM Complications Study. Diabet Med 2002; 19 : 900-909.

28. Pop-Busui R, Evans GW, Gerstein HC, Fonseca V, Fleg JL, Hoogwerf BJ, Genuth S, Grimm RH, Corson MA, Prineas R. Effects of cardiac autonomic dysfunction on mortality risk in the action to control cardiovascular risk in diabetes (ACCORD) trial. Diabetes Care 2010; 33: 1578-1584.

29. Boulton AJ, Vinik AI, Arezzo JC, Bril V, Feldman EL, Freeman R, Malik RA, Maser RE, Sosenko JM, Ziegler D. Diabetic neuropathies: a statement by the American Diabetes Association. Diabetes Care 2005; 28: 956-962.

30. Low PA, Benrud-Larson LM, Sletten DM, Opfer-Gehrking TL, Weigand SD, O'Brien PC, Suarez GA, Dyck PJ. Autonomic symptoms and diabetic neuropathy: a population-based study. Diabetes Care 2004; 27: 2942-2947.

31. Witte DR, Tesfaye S, Chaturvedi N, Eaton SE, Kempler P, Fuller JH; EURODIAB Prospective Complications Study Group. Risk factors for cardiac autonomic neuropathy in type 1 diabetes mellitus. Diabetologia 2005; 48: 164167. 
32. Vinik AI, Erbas T, Casellini CM. Diabetic cardiac autonomic neuropathy, inflammation and cardiovascular disease. J Diabetes Investig 2013; 4: 4-18.

33. Orchard TJ, Loyd CE, Maser RE, Kuller LH. Why does diabetic autonomic neuropathy predict IDDM mortality? An analysis from the Pittsburgh Epidemiology of Diabetes Complications Study. Diabetes Res Clin Pract 1996; 34: $165-171$.

34. Ziegler D, Zentai CP, Perz S, Rathmann W, Haastert B, Döring A, Meisinger C. Prediction of mortality using measures of cardiac autonomic dysfunction in the diabetic and nondiabetic population: the MONICA/KORA Augsburg Cohort Study. Diabetes Care 2008; 31: 556-561.

35. Maser RE, Lenhard MJ. Cardiovascular autonomic neuropathy due to diabetes mellitus: clinical manifestations, consequences, and treatment. J Clin Endocrinol Metab 2005; 90: 5896-5903.

36. Vinik AI, Maser RE, Ziegler D. Autonomic imbalance: prophet of doom or scope for hope. Diabet Med 2011; 28: 643-651.

37. Wackers FJ, Young LH, Inzucchi SE, Chyun DA, Davey JA, Barrett EJ, Taillefer R, Wittilin SD, Heller GV, Filipchuk N, Enges S, Rather RE, Iskandrian AE. Detection of silent myocardial ischemia in asymptomatic diabetic dubjects: The DIAD study. Diabetes Care 2004; 27: 1954-1961.

38. Veglio M, Chinaglia A, Cavallo-Perin P. QT interval, cardiovascular risk factors and risk of death in diabetes. J Endocrinol Invest 2004; 27: 175-181.

39. Pop-Busui R, Boulton AJM, Feldman EL, Bril V, Freeman R, Malik RA, Sosenko JM, Ziegler D. Diabetic neuropathy: A position statement by the American Diabetes Association. Diabetes Care 2017; 40: 136-154.

40. Dyck PJ, Albers JW, Andersen H, Arezzo JC, Biessels GJ, Bril V, Feldman EL, Litchy WJ, O'Brien PC, Russell JW. Toronto Expert Panel on Diabetic Neuropathy. Diabetic polyneuropathies: update on research definition, diagnostic criteria and estimation of severity. Diabetes Metab Res Rev 2011; 27: 620-628.

41. Albers JW, Pop-Busui R. Diabetic neuropathy: mechanisms, emerging treatments, and subtypes. Curr Neurol Neurosci Rep 2014; 14: 473.

42. Ewing DJ, Martyn CN, Young RJ, Clarke BF. The value of cardiovascular autonomic function tests: 10 years experience in diabetes. Diabetes Care 1985; 8: 491-498.

43. Low PA. Prevalence of orthostatic hypotension. Clin Auton Res 2008; 18 Suppl 1: 8-13.

44. Low PA, Walsh JC, Huang CY, McLeod JC.The sympathetic nervous system in diabetic neuropathy. A clinical and pathological study. Brain 1975; 98: $341-356$.

45. Task Force of the European Society of Cardiology and the North American Society of Pacing and Electrophysiology. Heart rate variability: standards of measurement, physiological interpretation and clinical use. Circulation 1996; 93: $1043-1065$.

46. Pop-Busui R. Cardiac autonomic neuropathy in diabetes. A clinical perspective. Diabetes Care. 2010; 33: 434-441. 
47. Ang L, Jaiswal M, Martin C, Pop-Busui R. Glucose control and diabetic neuropathy: Lessons from recent large clinical trials. Cur Diab Rep 2014; 14: 528 .

48. Martin CL, Albers JW, Pop-Busui R. Neuropathy and related findings in the diabetes control and complications trial/epidemiology of diabetes interventions and complications study. Diabetes Care 2014; 37: 31-38.

49. Ziegler D, Keller J, Maier C, Pannek J; German Diabetes Association. Diabetic neuropathy. Exp Clin Endocrinol Diabetes 2014; 122: 406-415.

50. Bernardi L, Spallone V, Stevens M, Hilsted J, Frontoni S, Pop-Busui R, Ziegler D, Kempler P, Freeman R, Low P, Tesfaye S, Valensi P; Toronto Consensus Panel on Diabetic Neuropathy. Methods of investigation for cardiac autonomic dysfunction in human research studies. Diabetes Metab Res Rev 2011, 27: $654-664$.

51. The Consensus Committee of the American Autonomic Society and the American Academy of Neurology. Consensus statement on the definition of orthostatic hypotension, pure autonomic failure, and multiple system atrophy. Neurology 1996; 46: 1470.

52. Spallone V, Bellarvere F, Scionti L, Maule S, Quadri R, Bax G, Megla P, Viviani GL, Esposito K, Morganti R, Cornelli P. Recommendations for the use of cardiovascular tests in diagnosing diabetic autonomic neuropathy. Nutr Metab Cardiovascular Dis 2011; 21: 69-78.

53. Hage FG, Iskandrian AE. Cardiovascular imaging in diabetes mellitus. J Nucl Cardiol 2011; 18: 959-965.

54. Valensi P, Johnson NB, Maison-Blanche P, Extramania F, Motte G, Coumel P. Influence of cardiac autonomic neuropathy on heart rate dependence of ventricular repolarization in diabetic patients. Diabetes Care 2002; 25: 918-923.

55. Santini V, Ciampittiello G, Gigli F, Bracaglia D, Baroni A, Cicconetti E, Verri C, Gambardella S, Frontosi S. QTc and autonomic neuropathy in diabetes: effects of acute hyperglycaemia and n-3 PUFA. Nutr Metab Cardiovasc Dis 2007; 17: 712-718.

56. Prince CT, Secrest AM, Mackey RH, Arena VC, Kingsley LA, Orchard TJ. Cardiovascular autonomic neuropathy, HDL cholesterol, and smoking correlate with arterial stiffness markers determined 18 years later in type 1 diabetes. Diabetes Care 2010; 33: 652-657.

57. Desouza CV, Bolli GB, Fonseca V. Hypoglycemia, diabetes, and cardiovascular events. Diabetes Care 2010; 33: 1389-1394.

58. Mancia G, De Backer G, Dominiczak A, Cifkova R, Fagard R, Germano G, Grassi G, Heagerty AM, Kjeldsen SE, Laurent S, Narkiewich K, Ruiloppe L, RynkewicA, Schmielder RE, Struijker Bouldier HAJ, Zancheetti A. 2007 ESH-ESC Guidelines for the management of arterial hypertension: the task force for the management of arterial hypertension of the European Society of Hypertension (ESH) and of the European Society of Cardiology (ESC). J Hypertens 2007; 25: 1105-1087.

59. Shakespeare CF, Katritsis D, Crowther A, Cooper IC, Coltart JD, Webb-Peploe MV. Differences in autonomic nerve function in patients with silent and symptomatic myocardial ischaemia. Br Heart J 1994; 71: 22-29. 
60. Veglio M, Borra M, Stevens LK, Fuller JH, Perin PC. The relation between QTc interval prolongation and diabetic complications: the EURODIAB IDDM Complications Study Group. Diabetologia 1999; 42: 68-75.

61. Kahn JK, Sisson JC, Vinik AI. Prediction of sudden cardiac death in diabetic autonomic neuropathy. J Nucl Med 1988; 29: 1605-1606.

62. Stevens M, Dayanikli F, Raffel D, Allman K, Standford T, Feldman E, Wieland D, Corbett J, Schwaiger M. Scintigraphic assessment of regionalized defects in myocardial sympathetic intervation and blood flow regulation in diabetic patients with autonomic neuropathy. J Am Coll Cardiol 1988; 31: 1575-1584.

63. Suarez GA, Clark VM, Norell JE, Kottke TE, Callahan MJ, O'Brien PC, Low PA, Dyck PJ. Sudden cardiac death in diabetes mellitus: risk factors in the Rochester Diabetic Neuropathy Study. J Neurol Neurosurg Psychiatry 2005; 76: 240-245.

64. Burgos LG, Ebert TJ, Assiddao C, Turner LA, Pattison CZ, WangCheng R, Kampline JP. Increased intraoperative cardiovascular morbidity in diabetics with autonomic neuropathy. Anesthesiology 1989; 70: 591-597.

65. Rolim LC, de Souza JST, Dib SA. Tests for early diagnosis of cardiovascular autonomic neuropathy: critical analysis and relevance. Front Endocrinol (Lausanne) 2013; 4: 173.

66. Bernardi L. Clinical evaluation of arterial baroreflex activity in diabetes. Diabetes Nutr Metab 2000; 13: 331-340.

67. Freeman R, Saul JP, Roberts MS, Berger RD, Broadbridge C, Cohen RJ. Spectral analysis of heart rate in diabetic autonomic neuropathy. A comparison with standard tests of autonomic function. Arch Neurol 1991; 48: 185-190.

68. Heart rate variability: standards of measurement, physiological interpretation and clinical use. Task Force of the European Society of Cardiology and the North American Society of Pacing and Electrophysiology. Circulation 1996; 93: 1043-1065.

69. Mogensen UM, JensenT, Kober L, Kelbaek H, Mathiesen AS, Dixen U, Rossing P, Hilsted J, Kofoed KF. Cardiovascular autonomic neuropathy and subclinical cardiovascular disease in normoalbuminuric Type 1 diabetic patients. Diabetes 2012; 61: 1822-1830.

70. Sammito S, Böckelmann I. Reference values for time- and frequencydomain heart rate variability measures. Heart Rhythm 2016; 1309-1316.

71. Bauer A,Malik M, Schmidt G, Barthel P, Bonnemeier H, Cygankiewicz I, Gusik P, Lombardi F, Muller A, Oto A, Schneider R, Watanabe M, Wichterle D, Zareba W. Heart rate turbulence: standards of measurement, physiological interpretation, and clinical use: International Society for Holter and Noninvasive Electrophysiology Consensus. J Am Coll Cardiol 2008; 52: 1353-1365.

72. Balc1oğlu AS, Müderrisoğlu H. Diabetes and cardiac autonomic neuropathy: Clinical manifestations, cardiovascular consequences, diagnosis and treatment. World J Diabetes 2015; 6: 80-91.

73. La Rovere MT, Pinna GD, Maestri R. et al. Prognostic implications of baroreflex sensitivity in heart failure patients in the beta-blocking era. J Am Coll Cardiol 2009; 53: 193-199.

74. Rosengård-Bärlund M, Bernardi L, Fagerudd J, Mäntysaari M,Af Björkesten CG,Lindholm H, Forsblom C, Waden J, Groop PH. FinnDiane Study 
Group. Early autonomic dysfunction in type 1 diabetes: a reversible disorder? Diabetologia 2009; 52: 1164-1172.

75. Hoffman RP, Sinkey CA, Anderson EA. Microneurographically determined muscle sympathetic nerve activity levels are reproducible in insulindependent diabetes mellitus. J Diabetes Complications 1998; 12: 307-310.

76. Hilsted J. Catecholamines and diabetic autonomic neuropathy. Diabet Med 1995; 12: 296-297.

77. Freeman MR, Newman D, Dorian P, Barr A, Langer A. Relation of direct assessment of cardiac autonomic function with metaiodobenzylguanidine imaging to heart rate variability in diabetes mellitus. Am J Cardiol 1987; 80: 247-250.

78. Schnell O, Muhr D, Weiss M, Dresel S, Haslbeck M, Standl E. Reduced myocardial 123I-metaiodobenzylguanidine uptake in newly diagnosed IDDM patients. Diabetes 1996; 45: 801-805.

79. Nagamachi S, Jinnouchi S, Kurose T, Ohnishi T, Flores LG 2nd, Nakahara H, Futami S, Tamura S, Matsukura S. 123I-MIBG myocardial scintigraphy in diabetic patients: relationship with 201Tl uptake and cardiac autonomic function. Ann Nucl Med 1998; 12: 323-331.

80. DeGrado TR, Hutchins GD, Toorongian SA, Wieland DM, Schwaiger M. Myocardial kinetics of carbon-11-meta-hydroxyephedrine (HED): retention mechanisms and effects of norepinephrine. J Nucl Med 1993; 34: 1287-1293.

81. Allman KC, Stevens MJ, Wieland DM, Hutchins GD, Wolfe ER Jr, Greene DA, Schwaiger M. Noninvasive assessment of cardiac diabetic neuropathy by C-11 hydroxyephedrine and positron emission tomography. J Am Coll Cardiol 1993; 22: 1425-1432.

82. Freeman R. Clinical practice. Neurogenic orthostatic hypotension. N Engl J Med 2008; 358: 615-624.

83. Low PA, Denq JC, Opfer-Gehrking TL, Dyck PJ, O'Brien PC, Slezak JM. Effect of age and gender on sudomotor and cardiovagal function and blood pressure response to tilt in normal subjects. Muscle Nerve 1997; 20: 1561-1568.

84. Pop-Busui R, Stevens M. Autonomic neuropathy in diabetes. In Therapy for Diabetes Mellitus and Related Disorders. 6th ed. Umpierrez GE, Ed. Alexandria, VA, American Diabetes Association 2014; 834-863.

85. Ziegler D, Voss A, Rathmann W, Strom A, Perz S, Roden M, Peters A, Meisinger C; KORA Study Group. Increased prevalence of cardiac autonomic dysfunction at different degrees of glucose intolerance in the general population: the KORA S4 survey. Diabetologia 2015; 58: 1118-1128.

86. Lykke JA, TarnowL, Parving HH, Hilsted J. A combined abnormality in heart rate variation and QT corrected interval is a strong predictor of cardiovascular death in type 1 diabetes. Scand J Clin Lab Invest 2008; 68: 654659.

87. Pop-Busui R. What do we know and we do not know about cardiovascular autonomic neuropathy in diabetes. J Cardiovasc Transl Res 2012; 5: $463-478$.

88. Ziegler D, Gries FA, Mühlen H, Rathmann W, Spuler M, Lessmann F. Prevalence and clinical correlates of cardiovascular autonomic and peripheral 
diabetic neuropathy in patients attending diabetes centers. Diabetes Metab 1993; 19: $143-151$.

89. Serhiyenko VA, Serhiyenko AA. Diabetic cardiac autonomic neuropathy: Do we have any treatment perspectives? World J Diabetes 2015; 6: 245-258.

90. Esposito K, Giugliano D. Mediterranean diet and type 2 diabetes. Diabetes Metab Res Rev 2014; 30: Suppl 1: 34-40.

91. Sleiman D, Al-Badri MR, Azar ST. Effect of Mediterranean diet in diabetes control and cardiovascular risk modification: A systematic review. Front Public Health 2015; 3: 69.

92. Carnethon MR, Prineas RJ, Temprosa M, Zhang ZM, Uwaifo G, Molitch ME; Diabetes Prevention Program Research Group. The association among autonomic nervous system function, incident diabetes, and intervention arm in the Diabetes Prevention Program. Diabetes Care 2006; 29: 914-919.

93. Smith AG, Russell J, Feldman EL, Goldstein J, Peltier A, Smith S, Hamwi J, Pollari D, Bixby B, Howard J, Singleton JR. Lifestyle intervention for pre-diabetic neuropathy. Diabetes Care 2006; 29: 1294-1299.

94. Balducci S, Iacobellis G, Parisi L, Di Biase N, Calandriello E, Leonetti F, Fallucca F. Exercise training can modify the natural history of diabetic peripheral neuropathy. J Diabetes Complications 2006; 20: 216-223.

95. Singleton JR, Marcus RL, Jackson JE, K Lessard M, Graham TE, Smith AG. Exercise increases cutaneous nerve density in diabetic patients without neuropathy. Ann Clin Transl Neurol 2014; 1: 844-849.

96. Colberg SR, Sigal RJ, Fernhall B, Regensteiner JG, Blissmer BJ, Rubin RR, Chasan-Taber L, Albright AI, Braun B. Exercise and Type 2 Diabetes: The American College of Sports Medicine and the American Diabetes Association: Joint position statement. Diabetes Care 2010; 33: 2692-2696.

97. Colberg SR, Vinik AI. Exercising with peripheral or autonomic neuropathy: what health care providers and diabetic patients need to know. Phys Sportmed 2014; 42: 15-23.

98. Pagkalos M, Koutlianos N, Kouidi E, Pagkalos E, Mandroukas K, Deligiannis A.Heart rate variability modifications following exercise training in type 2 diabetic patients with definite cardiac autonomic neuropathy. $\mathrm{Br} \mathrm{J}$ Sports Med 2008; 42: 47-54.

99. Epidemiology of Diabetes Interventions and Complications (EDIC). Design, implementation, and preliminary results of a long-term follow-up of the Diabetes Control and Complications Trial cohort. Diabetes Care 1999; 22: 99-111.

100. DCCT/EDIC, Writing Group. Sustained effect of intensive treatment of type 1 diabetes mellitus on development and progression of diabetic nephropathy: the Epidemiology of Diabetes Interventions and Complications (EDIC) study. JAMA 2003; 290: 2159-2167.

101. Cefalu WT, Ratner RE. The diabetes control and complications trial/ epidemiology of diabetes interventions and complications study at 30 years: the "gift" that keeps on giving! Diabetes Care 2014; 37: 5-7.

102. DCCT. The effect of intensive treatment of diabetes on the development and progression of long-term complications in insulin-dependent diabetes mellitus. 
The Diabetes Control and Complications Trial Research Group. N Engl J Med 1993; 329: 977-986.

103. DCCT. Effect of intensive diabetes treatment on nerve conduction in the Diabetes Control and Complications Trial. Ann Neurol 1995; 38: 869-880.

104. Pop-Busui R, Herman WH, Feldman EL, Low PA, Martin CL, Cleary PA, Waberski BH, Lachin JM, Albers JW. DCCTand EDIC studies in type 1 diabetes: lessons for diabetic neuropathy regarding metabolic memory and natural history. Curr Diab Rep 2010; 10: 276-282.

105. DCCT. The effect of intensive diabetes therapy on measures of autonomic nervous system function in the Diabetes Control and Complications Trial (DCCT). Diabetologia 1998; 41: 416-423.

106. Nathan DM, Cleary PA, Backlund JY, Genuth SM, Lachin JM, Orchard TJ, Raskin P, Zinman B. Intensive diabetes treatment and cardiovascular disease in patients with type 1 diabetes. N Engl J Med 2005; 353: 2643-2653.

107. Ohkubo Y, Kishikawa H, Araki E, Miyata T, Isami S, Motoyoshi S, Kojima Y, Furuyoshi N, Shichiri M. Intensive insulin therapy prevents the progression of diabetic microvascular complications in Japanese patients with noninsulin-dependent diabetes mellitus: a randomized prospective 6-year study. Diabetes Res Clin Pract 1995; 28: 103-117.

108. UKPDS. Intensive blood-glucose control with sulphonylureas or insulin compared with conventional treatment and risk of complications in patients with type 2 diabetes (UKPDS 33). UK Prospective Diabetes Study (UKPDS) Group. Lancet 1998; 352: 837-853.

109. Holman RR, Paul SK, Bethel MA, Matthews DR, Neil HA. 10-year follow-up of intensive glucose control in type 2 diabetes. N Engl J Med 2008; 359: $1577-1589$.

110. Duckworth W, Abraira C, Moritz T, Reda D, Emanuele N, Reaven PD, Zieve FJ, Franklin J, Marks J, Davis SN, Hayward R, Warren SR, Goldman S, McCarren M, Vitek ME, Henderson WG, Huang GD, forthe VADT Investigators. Glucose control and vascular complications in veterans with type 2 diabetes. N Engl J Med 2009; 360: 129-139.

111. Albers JW, Herman WH, Pop-Busui R, Feldman EL, Martin CL, Cleary PA, Waberski BH, Lachin JH and for the DCCT/EDIC Research Group. Effect of prior intensive insulin treatment during the Diabetes Control and Complications Trial (DCCT) on peripheral neuropathy in type 1 diabetes during the Epidemogy of Diabetes Interventions and Complications (EDIC) Study. Diabetes Care 2010; 33: 1090-1096.

112. Ismail-Beigi F, Craven T, Banerji MA, Basile J, Calles J, Cohen RM, Cuddihy R, Cushman WC, Genuth S, Gromm RH Jr, Hamilton BP, Hoogwert B, Karl D, Katz L, Krikonian A, O'Connor P, Pop-Busui R, Schubart U, Simmonds D, Taylor H, Thomas A, Weiss D, Hramiak I; ACCORD trial group. Effect of intensive treatment of hyperglycaemia on microvascular outcomes in type 2 diabetes: an analysis of the ACCORD randomised trial. Lancet 2010; 376: 419430 .

113. Charles M, Fleischer J, Witte DR, Ejskjaer N, Borch-Johnsen K, Lauritzen T, Sandbaek A. Impact of early detection and treatment of diabetes on the 6-year prevalence of cardiac autonomic neuropathy in people with screen- 
detected diabetes: ADDITION-Denmark, a cluster-randomised study. Diabetologia 2013; 56: 101-118.

114. Charles M, Ejskjaer N, Witte DR, Borch-Johnsen K, Lauritzen T, Sandbaek A. Prevalence of neuropathy and peripheral arterial disease and the impact of treatment in people with screen-detected type 2 diabetes: the ADDITIONDenmark study. Diabetes Care 2011; 34: 2244-2249.

115. Catapano AL, Graham I, De Backer G, Wiklund O, Chapman MJ, Drexel H, Hoes AW, Jennings CS, Landmesser U, Pedersen TR, Reiner Z, Riccardi G, Taskinen M-R, Tokgozoglu L, Verschuren WMM, Vlachopoulos C, Wood DA, Zamorano JL, Authors/Task Force Members; 2016 ESC/EAS Guidelines for the Management of Dyslipidaemias. Europ Heart J 2016; 37: 2999-3058.

116. Colhoun HM, Betteridge DJ, Durrington PN, Hitman GA, Neil HA, Livigstone SJ, Thomason MJ, Mackness MI, Charlton-Menys V, Fuller JH; CARDS investigators. Primary prevention of cardiovascular disease with atorvastatin in type 2 diabetes in the Collaborative Atorvastatin Diabetes Study (CARDS): multicentre randomised placebo-controlled trial. Lancet 2004; 364: 685-696.

117. Jellinger PS, Handelsman Y, Rosenblit PD, Bloomgarden ZT, Fonseca VA, Garber AJ, Grunberger G, Guerin CK, Bell DSH, Mechanick JI, PessahPollack R, Wyne K, Smith D, Brinton EA, Fazio S, Davidson M. American Association of Clinical Endocrinologists and American College of Endocrinology Guidelines for management of dyslipidemia and prevention of cardiovascular disease. Endocr Pract 2017; 23: S1-S86.

118. Abifadel M, Varret M, Rabes JP, Allard D, Ouguerram K, Devillers M, Cruaud C, Benjannet S, Wickham L, Erlich D, Derre A, Villeger L, Farnier M, Beucler I, Bruckert E, Chambaz J, Chanu B, Lecerf JM, Luc G, Moulin P, Weissenbach J, Prat A, Krempf M, Junien C, Seidah NG, Boileau C. Mutations in PCSK9 cause autosomal dominant hypercholesterolemia. Nat Genet 2003; 34: 154-156.

119. Praluent (alirocumab) prescribing information. Bridgewater, NJ: SanofiAventis US; 2015.

120. Raal FJ, Stein EA, Dufour R, Turner T, Civeira F, Burgess L, Langslet G, Scott R, Olsson AG, Sullivan D, Hovingh GK, Cariou B, Gouni-Berthold I, Somarathe R, Bridges I, Scott R, Wasserman SM, Gaudet D. PCSK9 inhibition with evolocumab (AMG 145) in heterozygous familial hypercholesterolaemia (RUTHERFORD-2): a randomised, double-blind, placebo-controlled trial. Lancet 2015; 385: 331-340.

121. Robinson JG, Farnier M, Krempf M, Bergeron J, Luc G, Averna M, Stroes ES, Langslet G. Efficacy and safety of alirocumab in reducing lipids and cardiovascular events. N Engl J Med 2015; 372: 1489-1499.

122. Stoekenbroek RM, Kastelein JJ, Huijgen R. PCSK9 inhibition: the way forward in the treatment of dyslipidemia. BMC Med 2015; 13: 258.

123. Schwartz GG, Bessac L, Berdan LG, Bhatt DL, Bittner V, Diaz R, Goodman SG. Effect of alirocumab, a monoclonal antibody to PCSK9, on longterm cardiovascular outcomes following acute coronary syndromes: rationale and design of the ODYSSEY outcomes trial. Am Heart J 2014; 168: 682-689.

124. Norata GD, Tibolla G, Catapano AL. Targeting PCSK9 for hypercholesterolemia. Annu Rev Pharmacol Toxicol 2014; 54: 273-293. 
125. Chapman MJ, Redfern JS, McGovern ME, Giral P. Niacin and fibrates in atherogenic dyslipidemia: pharmacotherapy to reduce cardiovascular risk. Pharmacol Ther 2010; 126: 314-345.

126. Thompson A, Danesh J. Associations between apolipoprotein B, apolipoprotein AI, the apolipoprotein B/AI ratio and coronary heart disease: a literature-based meta-analysis of prospective studies. J Intern Med 2006; 259: 481-492.

127. AIM-HIGH Investigators, Boden WE, Probstfield JL, Anderson T, Chaitman BR, Desvignes-Nickens P, Koprowicz K, McBride R, Teo K, Weintraub W. Niacin in patients with low HDL cholesterol levels receiving intensive statin therapy. N Engl J Med 2011; 365: 2255-2267.

128. HPS2-THRIVE Collaborative Group, Landray MJ, Haynes R, Hopewell JC, Parish S, Aung T, Tomson J, Wallendszus K, Craig M, Jiang L, Collins R, Armitage J. Effects of extended-release niacin with laropiprant in high-risk patients. N Engl J Med 2014; 371: 203-212.

129. Ooi CP, Loke SC. Colesevelam for type 2 diabetes mellitus: an abridged Cochrane review. Diabet Med 2014; 31: 2-14.

130. Ahn $\mathrm{CH}$, Choi SH. New drugs for treating dyslipidemia: Beyond statins. Diabetes Met 2015; 39: 87-94.

131. Bell DA, Hooper AJ, Burnett JR. Mipomersen, an antisense apolipoprotein B synthesis inhibitor. Expert Opin Invesg Drugs 2011; 20: 265272.

132. Adiels M, Olofsson SO, Taskinen MR, Boren J. Overproduction of very low density lipoproteins is the hallmark of the dyslipidemia in the metabolic syndrome. Arterioscler Thromb Vasc Biol 2008; 28: 1225-1236.

133. Scott R, O'Brien R, Fulcher G, Pardy C, D'Emden M, Tse D, Taskinen MR, Ehnholm C, Keech A. Effects of fenofibrate treatment on cardiovascular disease risk in 9,795 individuals with type 2 diabetes and various components of the metabolic syndrome: the Fenofibrate Intervention and Event Lowering in Diabetes (FIELD) study. Diabetes Care 2009; 32: 493-498.

134. Taskinen MR, Boren J. New insights into the pathophysiology of dyslipidemia in type 2 diabetes. Atherosclerosis 2015; 239: 483-495.

135. Cholesterol Treatment Trialists' (CTT) Collaboration, Baigent C, Blackwell L, Emberson J, Holland LE, Reith C, Bhala N, Peto R, Barnes EH, Keech A, Simes J, Collins R. Efficacy and safety of more intensive lowering of LDL cholesterol: a meta-analysis of data from 170,000 participants in 26 randomised trials. Lancet 2010; 376: 1670-1681.

136. Sattar N, Preiss D, Murray HM, Welsh P, Buckley BM, de Craen AJ, Seshasai SR, McMurray JJ, Freeman DJ, Jukema JW, Macfarlane PW, Packard CJ, Stott DJ, Westendorp RG, Shepherd J, Davis BR, Pressel SL, Marchioli R, Marfisi RM, Maggioni AP, Tavazzi L, Tognoni G, Kjekshus J, Pedersen TR, Cook TJ, Gotto AM, Clearfield MB, Downs JR, Nakamura H, Ohashi Y, Mizuno K, Ray KK, Ford I. Statins and risk of incident diabetes: a collaborative meta-analysis of randomised statin trials. Lancet 2010; 375: 735-742.

137. Keech A, Simes RJ, Barter P, Best J, Scott R, Taskinen MR, Forder P, Pillai A, Davis T, Glasziou P, Drury P, Kesa.niemi YA, Sullivan D, Hunt D, Colman P, d'Emden M, Whiting M, Ehnholm C, Laakso M. Effects of long-term 
fenofibrate therapy on cardiovascular events in 9795 people with type 2 diabetes mellitus (the FIELD study): randomised controlled trial. Lancet 2005; 366: 18491861.

138. ACCORD Study Group, Ginsberg HN, Elam MB, Lovato LC, Crouse JR 3rd, Leiter LA, Linz P, Friedewald WT, Buse JB, Gerstein HC, Probstfield J, Grimm RH, Ismail-Beigi F, Bigger JT, Goff DC Jr, Cushman WC, Simons-Morton DG, Byington RP. Effects of combination lipid therapy in type 2 diabetes mellitus. N Engl J Med 2010; 362: 1563-1574.

139. Saha SA, Arora RR. Fibrates in the prevention of cardiovascular disease in patients with type 2 diabetes mellitus - a pooled meta-analysis of randomized placebo controlled clinical trials. Int J Cardiol 2010; 141: 157-166.

140. Collins R, Armitage J, Parish S, Sleigh P, Peto R; Heart Protection Study Collaborative Group. MRC/BHF Heart Protection Study of cholesterollowering with simvastatin in 5963 people with diabetes: a randomised placebocontrolled trial. Lancet 2003; 361: 2005-2016.

141. Brugts JJ, Yetgin T, Hoeks SE, Gotto AM, Shepherd J, Westendorp RG, de Craen AJ, Knopp RH, Nakamura H, Ridker P, van Domburg R, Deckers JW. The benefits of statins in people without established cardiovascular disease but with cardiovascular risk factors: meta-analysis of randomised controlled trials. BMJ 2009; 338: b2376.

142. Power RA, Hulver MW, Zhang JY, Dubois J, Marchand RM, Ilkayeva O, Muoio DM, Mynatt RL. Carnitine revisited: potential use as adjunctive treatment in diabetes. Diabetologia 2007; 50: 824-832.

143. Solfrizzi V, Capurso C, Colacicco AM, D'Introno A, Fontana C, Capurso SA, Torres F, Gadaleta AM, Koverech A, Capurso A, et al. Efficacy and tolerability of combined treatment with L-carnitine and simvastatin in lowering lipoprotein(a) serum levels in patients with type 2 diabetes mellitus. Atherosclerosis 2006; 188: 455-461.

144. Kasznicki J, Kosmalski M, Sliwinska A, Mrowicka M, Stanczyk M, Majsterek I, Drzewoski J. Evaluation of oxidative stress markers in pathogenesis of diabetic neuropathy. Mol Biol Rep 2012; 39: 8669-8678.

145. Oyenihi AB, Ayeleso AO, Mukwevho E, Masola B. Antioxidant strategies in the management of diabetic neuropathy. Biomed Res Int 2015; 2015: 15 .

146. Bandeira S de M, da Fonseca LJS, Guedes G da S, Rabelo LA, Goulart MOF, Vasconcelos SML. Oxidative stress as an underlying contributor in the development of chronic complications in diabetes mellitus. Int J Mol Sci 2013; 14: $3265-3284$.

147. Iwaki M, Yamada Y, Nakajima Y, Nakayama O, Makishima M, Matsuda M, Shimomura I. Increased oxidative stress in obesity and its impact on metabolic syndrome. J Clin Invest 2004; 114: 1752-1761.

148. Vincent AM, Russell JW, Low P, Feldman EL. Oxidative stress in the pathogenesis of diabetic neuropathy. Endocr Rev 2004; 25: 612-628.

149. Obrosova IG, Ilnytska O, Lyzogubov VV, Pavlov IA, Mashtair N, Nadler JL, Drel VR. High-fat diet-induced neuropathy of pre-diabetes and obesity: effects of "healthy" diet and aldose reductase inhibition. Diabetes 2007; 56: 25982608. 
150. Negre-Salvayre A, Coatrieux C, Ingueneau C, Salvayre R. Advanced lipid peroxidation end products in oxidative damage to proteins. Potential role in diseases and therapeutic prospects for the inhibitors. Br J Pharmacol 2008; 153: 6-20.

151. Mahmood D, Singh BK, Akhtar M. Diabetic neuropathy: therapies on the horizon. J Pharm Pharmacol 2009; 61: 1137-1145.

152. Kalousov'a M, Skrha J, Zima T. Advanced glycation endproducts and advanced oxidation protein products in patients with diabetes mellitus. Physiol Res 2002; 51: 597-604.

153. King RHM. The role of glycation in the pathogenesis of diabetic polyneuropathy. Mol Pathol 2001; 54: 400-408.

154. Valko M, Leibfritz D, Moncol J, Cronin MTD, Mazur M, Telser J. Free radicals and antioxidants in normal physiological functions and human disease. Int J Biochem Cell Biol 2007; 39: 44-84.

155. Bajaj S, Khan A. Antioxidants and diabetes. Indian J Endocrinol Metab 2012; 16: 267-271.

156. Balarabe SA, Adamu MD, Watila MM, Jiya N. Neuromyelitis optica and myasthenia gravis in a young Nigerian girl. BMJ Case Rep 2015.

157. Hamilton SJ, Chew GT, Watts GF. Therapeutic regulation of endothelial dysfunction in type 2 diabetes mellitus. Diab Vasc Dis Res 2007; 4: 89-102.

158. Packer L, Kraemer K, Rimbach G. Molecular aspects of lipoic acid in the prevention of diabetes complications. Nutrition 2001; 17: 888-895.

159. Ziegler D, Schatz H, Conrad F, Gries FA, Ulrich H, Reichel G. Effects of treatment with the antioxidant alpha-lipoic acid on cardiac autonomic neuropathy in NIDDM patients. A 4-month randomized controlled multicenter trial (DEKAN Study). Deutsche Kardiale Autonome Neuropathie. Diabetes Care 1997; 20: 369373.

160. Bertolotto F, Massone A. Combination of alpha lipoic acid and superoxide dismutase leads to physiological and symptomatic improvements in diabetic neuropathy. Drugs R D 2012; 12: 29-34.

161. McNulty H, Jacob RF, Mason RP. Biologic activity of carotenoids related to distinct membrane physicochemical interactions. Am J Cardiol 2008; 22: $20-29$.

162. Berrone E, Beltramo E, Solimine C, Ape AU, Porta M. Regulation of intracellular glucose and polyol pathway by thiamine and benfotiamine in vascular cells cultured in high glucose. J Biol Chem 2006; 281: 9307-9313.

163. Lazo de la Vega-Monroy ML, Larrieta E, German MS, Baez-Saldana A, Fernandez-Mejia C. Effects of biotin supplementation in the diet on insulin secretion, islet gene expression, glucose homeostasis and beta-cell proportion. $\mathrm{J}$ Nutr Biochem 2013; 24: 169-177.

164. Sheikh-Ali M, Chehade JM, Mooradian AD. The antioxidant paradox in diabetes mellitus. Am J Ther 2011; 18: 266-278.

165. Rahimi R, Nikfar S, Larijani B, Abdollahi M. A review on the role of antioxidants in the management of diabetes and its complications. Biomed Pharmacother 2005; 59: 365-373.

166. Hotta N, Akanuma Y, Kawamori R, Matsuoka K, Oka Y, Shichiri M, Toyota T, Nakashima M, Yoshimura I, Sakamoto N, Shigeta Y. Long-term clinical 
effects of epalrestat, an aldose reductase inhibitor, on diabetic peripheral neuropathy: The 3-year, multicenter, comparative aldose reductase inhibitor-diabetes complications trial. Diabetes Care 2006; 29: 1538-1544.

167. Haupt E, Ledermann H, Köpcke W. Benfotiamine in the treatment of diabetic polyneuropathy-a three-week randomized, controlled pilot study (BEDIP study). Int J Clin Pharmacol Ther 2005; 43: 71-77.

168. Stracke H, Gaus W, Achenbach U, Federlin K, Bretzel RG. Benfotiamine in Diabetic Polyneuropathy (BENDIP): Results of a Randomised, Double Blind, Placebo-controlled Clinical Study. Exp Clin Endocrinol Diabetes 2008; 116: 600-605.

169. Balakumar P, Rohilla A, Krishan P, Solairaj P, Thangathirupathi A. The multifaceted therapeutic potential of benfotiamine. Pharmacol Res 2010;61: 482-488.

170. Stracke H, Lindemann A, Federlin K. A benfotiamine-vitamin B combination in treatment of diabetic polyneuropathy. Exp Clin Endocrinol Diabetes 1996; 104: 311-316.

171. Lonsdale D. Dysautonomia, a heuristic approach to a revised model for etiology of disease. J Evid Based Complementary Altern Med 2009; 6: 3-10.

172. Lonsdale D, Shamberger RJ, Obrenovich ME. Exaggerated autonomic asymmetry: A clue to nutrient deficiency dysautonomia. WebmedCentral ALTERNATIVE MEDICINE 2011; 2: Article ID WMC001854, 13 pages.

173. Barclay LL, Gibson GE, Blass JP. Impairment of behavior and acetylcholine metabolism in thiamine deficiency. J Pharmacol Ex Ther 1981; 217: 537-543.

174. Johnson TS, Young JB, Landsberg L. Sympathoadrenal responses to acute and chronic hypoxia in the rat. J Clin Invest 1983; 71: 1263-1272.

175. Matthews DR, Hosker JP, Rudenski AS, et al. Homeostasis model assessment: insulin resistance and betacell function from fasting plasma glucose and insulin concentrations in man. Diabetologia 1985; 28: 412-419.

176. Bazett HC. An analysis of the time-relations of electrocardiograms. Ann Noninvasive Electrocardiol. 1997; 2: 177-194.

177. Borleffs CJW, Scherptong RWC, Man S-C, et al. Predicting ventricular arrhythmias in patients with ischemic heart disease: clinical application of the ECG-derived QRS-T angle. Circ Arrhythm Electrophysiol 2009; 2: 548-554.

178. TensioClinic TensioMed ${ }^{\mathrm{TM}}$ Arteriograph and TensioMed ${ }^{\mathrm{TM}}$ program. Available from: URL: http//www.arteriograph.nl/?act=download\&fid=173.

179. Polizzi C, Andican G, Cetin E, et al. Increased DNA-glycation in type 2 diabetic patients: the effect of thiamine and pyridoxine therapy. Exp Clin Endocrinol Diabetes 2012; 120: 329-334.

180. Valdes-Ramos R, Guadarrama-Lopez AL, Martinez-Carrillo BE, et al. Vitamins and type 2 diabetes mellitus. Endocr Metab Immune Disord Drug Targets 2015; 15: 54-63.

181. Thornalley PJ, Babaei-Jadidi H, Rabbani N, et al. High prevalence of low plasma thiamine concentration in diabetes linked to a marker of vascular disease. Diabetologia 2007; 50: 164-217. 
182. Babaei-Jadidi R, Karachalias N, Kupich C, et al. High-dose thiamine therapy counters dyslipidaemia in streptozotocin-induced diabetic rats. Diabetologia 2004; 47: 2235-2246.

183. Pácal L, Kuricová K, Kaňková K. Evidence for altered thiamine metabolism in diabetes: is there a potential to oppose gluco- and lipotoxicity by rational supplementation? World J Diabetes 2014; 5: 288-295.

184. Moss CJ, Mathews ST. Thiamin status and supplementation in the management of diabetes mellitus and its vascular comorbidities. Vitamins \& Minerals 2013; 2: 111.

185. Stirban A, Pop A, Tschoepe D. A randomized, double-blind, crossover, placebo-controlled trial of 6 weeks benfotiamine treatment on postprandial vascular function and variables of autonomic nerve function in Type 2 diabetes. Diabet Med 2013; 30: 1204-1208.

186. Ziegler D, Schleicher E, Strom A, et al. Association of transketolase polymorphisms with measures of polyneuropathy in patients with recently diagnosed diabetes. Diabetes Metab Res Rev 2016; 33: e2811.

187. Spallone V. Might genetics play a role in understanding and treating diabetic polyneuropathy? Diabetes Metab Res Rev 2017; 33: 2882-2886.

188. Rabbani N, Thornalley PJ. Glyoxalase in diabetes, obesity and related disorders. Seminars in Cell \& Developmental Biology 2011; 22: 309-317.

189. Luong KV, Nguyen LT. The impact of thiamine treatment in the diabetes mellitus. J Clin Med Res 2012; 4: 153-160.

190. González-Ortiz M, Martínez-Abundis E, Robles-Cervantes JA, et al. Effect of thiamine administration on metabolic profile, cytokines and inflammatory markers in drug-naïve patients with type 2 diabetes. Europ J Nutr 2011; 50: 145149.

191. Katare RG, Caporali A, Emanueli C, Madeddu P. Benfotiamine improves functional recovery of the infarcted heart via activation of pro-survival G6PD/Akt signaling pathway and modulation of neurohormonal response. J Mol Cell Cardiol 2010; 49: 625-638.

192. Bitsch R, Wolf M, Möller J, Heuzeroth L, Grüneklee D. Bioavailability assessment of the lipophilic benfotiamine as compared to a water-soluble thiamin derivate. Ann Nutr Metab 1991; 35: 292-296.

193. Stracke H, Gaus W, Achenbach U, Federlin K, Bretzel RG. Benfotiamine in diabetic polyneuropathy (BENDIP): Results of a randomised, double blind, placebo-controlled clinical study. Exp Clin Endocrinol Diabetes 2008; 116: 600-605.

194. Rašić-Milutinović Z, Gluvić, Peruničić-Peković G, Miličević D, Lačković M, Penčić B. Improvement of heart rate variability with benfothiamine and alpha-lipoic acid in type 2 diabetic patients - Pilot study. J Cardiol Therap 2014; 2: 1-7.

195. Aronson D. Pharmacologic modulation of autonomic tone: implications for the diabetic patient. Diabetologia 1997; 40: 476-481.

196. Page L, Laight D, Cummings MH. Thiamine deficiency in diabetes mellitus and the impact of thiamine replacement on glucose metabolism and vascular disease. Int J Clin Pract 2011; 65: 684-690. 
197. Karachalias N, Babaei-Jadidi R, Rabbani N, Thornalley PJ. Increased protein damage in renal glomeruli, retina, nerve, plasma and urine and its prevention by thiamine and benfotiamine therapy in a rat model of diabetes. Diabetologia 2010; 52: 1506-1516.

198. Valensi P, Avignon A, Sultan A, et al. Atherogenic dyslipidemia and risk of silent coronary artery disease in asymptomatic patients with type 2 diabetes: a cross-sectional study. Cardiovasc Diabetol 2016; 15: 104.

199. Alkhalaf A, Klooster A, van Oeveren W, et al. A double-blind, randomized, placebo-controlled clinical trial on benfotiamine treatment in patients with diabetic nephropathy. Diabetes Care 2010; 33: 1598-1601.

200. Sergienko VA, Segin VB, Samir A, Sergienko AA. The effect of longchain polyunsaturated higher omega-3 fatty acids, benfotiamine and alpha-lipoic acid on the lipid metabolism in patients with diabetes mellitus type 2 and cardiovascular autonomic neuropathy. Zh Nevrol Psikhiatr Im SS Korsakova (Russian) 2013; 113: 54-58.

201. Bozic I, Savic D, Laketa D. et al. Benfotiamine attenuates inflammatory response in LPS stimulated BV-2 microglia. PLoS One 2015; 10: 23 pages. Article ID $\mathrm{e} 0118372$.

202. Yaday UC, Kalariya NM, Srivastava SK,. Ramana KV. Protective role of benfotiamine, a fat soluble vitamin B1 analogue, in the lipopolysaccharideinduced cytotoxic signals in murine macrophages. Free Radic Biol Med 2010; 48: $1423-1434$.

203. Shoeb M, Ramana KV. Anti-inflammatory effects of benfotiamine are mediated through the regulation of the arachidonic acid pathway in macrophages. Free Radic Biol Med 2012; 52: 182-190.

204. Serhiyenko AA, Serhiyenko LM, Serhiyenko VA. Effects of treatment with alpha-lipoic acid and benfotiamine in Type 1 diabetic patients with peripheral neuropathy. Diabetologia 2005; 48: Suppl 1, PS969.

205. Yenilmez M, Ozcifci M, Aydin Y, et al. Protective effect of high-dose thiamine (B1) on rat detrusor contractility in streptozotocin-induced diabetes mellitus. Acta Diabetologica 2006; 43: 103-108.

206. Stepuro AI, Adamchuk RI, Oparin A.Yu, Stepuro II. Thiamine inhibits formation of dityrosine, a specific marker of oxidative injury, in reactions catalyzed by oxoferryl forms of hemoglobin. Biochemistry (Moscow) 2008; 73: 1031-1041.

207. Gioda R, de Oliveira Barreto T, Primola-Gomes TN. et al. Cardiac oxidative stress is involved in heart failure induced by thiamine deprivation in rats. Am J Physiol Heart Circ Physiol 2010; 298: H2039-H2045.

208. Verma S, K. Reddy K, Balakumar P. The defensive effect of benfotiamine in sodium arsenite-induced experimental vascular endothelial dysfunction. Biol Trace Elem Res 2010; 137: 96-109.

209. Valensi P, Cosson E. Diabetic cardiovascular autonomic neuropathy. Eur J Endocrinol 2007; 2: 69-71.

210. Yan H, Guo Y, Zhang J, Ding Z, Ha W, Harding JJ. Effect of carnosine, aminoguanidine, and aspirin drops on the prevention of cataracts in diabetic rats. Mol Vis 2008; 14: 2282-2291. 
211. Urios P, Grigorova-Borsos A-M, Sternberg M. Aspirin inhibits the formation of pentosidine, a cross-linking advanced glycation end product, in collagen. Diabetes Res Clin Pract 2007; 77: 337-340.

212. Shen Q, Pierce JD. Supplementation of Coenzyme Q10 among patients with type 2 diabetes mellitus. Healthcare (Basel) 2015; 3: 296-309.

213. Burnier M. Telmisartan: a different angiotensin II receptor blocker protecting a different population? J Int Med Res 2009; 37: 1662-1679.

214. Ahmed MA, Muntingh GL, Rheeder P. Perspectives on peripheral neuropathy as a consequence of metformin-induced vitamin B12 deficiency in T2DM. Int J Endocrinol 2017; 2017: 2452853.

215. Fragasso G, Palloshi A, Puccetti P, Silipigni C, Rossodivita A, Pala $\mathrm{M}$, Calori G, Alfieri O, Margonato A. A randomized clinical trial of trimetazidine, a partial free fatty acid oxidation inhibitor, in patients with heart failure. J Am Coll Cardiol 2006; 48: 992-998.

216. Lee W-S, Kim J. Diabetic cardiomyopathy: where we are and where we are going. Korean J Intern Med 2017; 32: 404-421.

217. Lee L, Campbell R, Scheuermann-Freestone M, Taylor R, Gunaruwan P, Williams L, Ashrafian H, Horowitz J, Fraser AG, Clarke K, Frenneaux M. Metabolic modulation with perhexiline in chronic heart failure: a randomized, controlled trial of short-term use of a novel treatment. Circulation 2005; 112: 3280-3288.

218. Morrow DA, Scirica BM, Karwatowska-Prokopczuk E, Murphy SA, Budaj A, Varshavsky S, Wolff AA, Skene A, McCabe CH, Braunwald E. Effects of ranolazine on recurrent cardiovascular events in patients with non-ST-elevation acute coronary syndromes: the MERLIN-TIMI 36 randomized trial. JAMA 2007; 297: $1775-1783$.

219. Witteles RM, Fowler MB. Insulin-resistant cardiomyopathy clinical evidence, mechanisms, and treatment options. J Am Coll Cardiol 2008; 51: 93102.

220. Nikolaidis LA, Poornima I, Parikh P, Magovern M, Shen YT, Shannon RP. The effects of combined versus selective adrenergic blockade on left ventricular and systemic hemodynamics, myocardial substrate preference, and regional perfusion in conscious dogs with dilated cardiomyopathy. J Am Coll Cardiol 2006; 47: $1871-1881$.

221. Sytze Van Dam P, Cotter MA, Bravenboer B, Cameron NE. Pathogenesis of diabetic neuropathy: focus on neurovascular mechanisms. Eur $\mathrm{J}$ Pharmacol 2013; 719: 180-186.

222. Tzemos N, Lim PO, MacDonald TM. Nebivolol reverses endothelial dysfunction in essential hypertension: a randomized, double-blind, crossover study. Circulation 2001; 104: 511-514.

223. Zepeda RJ, Castillo R, Rodrigo R, Prieto JC, Aramburu I, Brugere S, Galdames K, Noriega V, Miranda HF. Effect of carvedilol and nebivolol on oxidative stress-related parameters and endothelial function in patients with essential hypertension. Basic Clin Pharmacol Toxicol 2012; 111: 309-316.

224. Su JB. Vascular endothelial dysfunction and pharmacological treatment. World J Cardiol 2015; 7: 719-741. 
225. Kelly AS, Gonzalez-Campoy JM, Rudser KD, Katz H, Metzig AM, Thalin M, Bank AJ. Carvedilol-lisinopril combination therapy and endothelial function in obese individuals with hypertension. J Clin Hypertens (Greenwich) 2012; 14: 85-91.

226. Mak IT, Boehme P, Weglicki WB. Antioxidant effects of calcium channel blockers against free radical injury in endothelial cells. Correlation of protection with preservation of glutathione levels. Circ Res 1992; 70: 1099-1103.

227. Matsubara M, Hasegawa K. Benidipine, a dihydropyridine calcium channel blocker, prevents lysophosphatidylcholine induced injury and reactive oxygen species production in human aortic endothelial cells. Atherosclerosis 2005; 178: $57-66$.

228. Habib JB, Bossaller C, Wells S, Williams C, Morrisett JD, Henry PD. Preservation of endothelium-dependent vascular relaxation in cholesterol-fed rabbit by treatment with the calcium blocker PN 200110. Circ Res 1986; 58: 305-309.

229. Fukao K, Shimada K, Hiki M, Kiyanagi T, Hirose K, Kume A, Ohsaka H, Matsumori R, Kurata T, Miyazaki T, Daida H. Effects of calcium channel blockers on glucose tolerance, inflammatory state, and circulating progenitor cells in non-diabetic patients with essential hypertension: a comparative study between azelnidipine and amlodipine on glucose tolerance and endothelial function -a crossover trial (AGENT). Cardiovasc Diabetol 2011; 10: 79.

230. Celık T, Balta S, Karaman M, Ahmet Ay S, Demırkol S, Ozturk C, Dinc M, Unal HU, Yilmaz MI, Kilıc S, Kurt G, Tas A, Iyısoy A, Quart1-Trevano F, Fic1 F, Grass1 G. Endocan, a novel marker of endothelial dysfunction in patients with essential hypertension: comparative effects of amlodipine and valsartan. Blood Press 2015; 24: 55-60.

231. Fukutomi M, Hoshide S, Mizuno H, Kario K. Differential effects of aliskiren/amlodipine combination and high-dose amlodipine monotherapy on endothelial function in elderly hypertensive patients. Am J Hypertens 2014; 27: 14-20.

232. Okamura T, Tawa M, Geddawy A, Shimosato T, Iwasaki H, Shintaku H, Yoshida Y, Masada M, Shinozaki K, Imamura T. Effects of atorvastatin, amlodipine, and their combination on vascular dysfunction in insulin-resistant rats. J Pharmacol Sci 2014; 124: 76-85.

233. Zhou MS, Tian R, Jaimes EA, Raij L. Combination therapy of amlodipine and atorvastatin has more beneficial vascular effects than monotherapy in salt-sensitive hypertension. Am J Hypertens 2014; 27: 873-880.

234. Jain NK, Patil CS, Singh A, Kulkarni SK. Sildenafil-induced peripheral analgesia and activation of the nitric oxide-cyclic GMP pathway. Brain Res 2001; 909: $170-178$.

235. Patil CS, Singh VP, Singh S, Kulkarni SK. Modulatory effect of the PDE-5 inhibitor sildenafil in diabetic neuropathy. Pharmacology 2004; 72: 190 195.

236. Wang L, Chopp M, Szalad A, Liu Z, Bolz M, Alvarez FM, Lu M, Zhang L, Cui Y, Zhang RL, Zhang ZG. Phosphodiesterase-5 is a therapeutic target for peripheral neuropathy in diabetic mice. Neuroscience 2011; 193: 399-410. 
237. Peixoto CA, Nunes AK, Garcia-Osta A. Phosphodiesterase-5 inhibitors: action on the signaling pathways of neuroinflammation, neurodegeneration, and cognition. Mediators Inflamm 2015; 2015: 940207.

238. Schafer A, Fraccarollo D, Pfortsch S, Flierl U, Vogt C, Pfrang J, Kobsar A, Renne T, Eigenthaler M, Ertl G, Bauersachs J. Improvement of vascular function by acute and chronic treatment with the PDE-5 inhibitor sildenafil in experimental diabetes mellitus. Br J Pharmacol 2008; 153: 886-893.

239. Wang L, Chopp M, Szalad A, Lu X, Jia L, Lu M, Zhang RL, Zhang ZG. Tadalafil promotes the recovery of peripheral neuropathy in type II diabetic mice. PloS One 2016; 11: e0159665.

240. Wang L, Chopp M, Zhang ZG. PDE5 inhibitors promote recovery of peripheral neuropathy in diabetic mice. Neural Regen Res 2017; 12: 218-219.

241. Tse S, Mazzola N. Ivabradine (Corlanor) for heart failure: The first selective and specific If inhibitor.PT 2015; 40: 810-814.

242. DiFrancesco D. The role of the funny current in pacemaker activity. Circ Res 2010; 106: 434-446.

243. Reil JC, Tardif JC, Ford I, Lloyd SM, O'Meara E, Komajda M, Borer JS, Tavazzi L, Swedberg K, Bohm M. Selective heart rate reduction with ivabradine unloads the left ventricle in heart failure patients. J Am Coll Cardiol 2013; 62: 1977-1985.

244. Rienzo M, Melka J, Bize A, Sambin L, Jozwiak M, Su JB, Hittinger L, Berdeaux A, Ghaleh B. Ivabradine improves left ventricular function during chronic hypertension in conscious pigs. Hypertension 2015; 65: 122-129.

245. Jochmann N, Schroter F, Knebel F, Hattasch R, Gericke C, Stangl K, Baumann G, Stangl V. Effect of ivabradine-induced heart rate reduction on flowmediated dilation measured with high sensitivity ultrasound in patients with stable coronary heart disease. Cardiovasc Ultrasound 2014; 12: 5.

246. Nerla R, Di Franco A, Milo M, Pitocco D, Zaccardi F, Tarzia P, Sarullo FM, Villano A, Russo G, Stazi A, Ghirlanda G, Lanza GA, Crea F. Differential effects of heart rate reduction by atenolol or ivabradine on peripheral endothelial function in type 2 diabetic patients. Heart 2012; 98: 1812-1816.

247. Fox K, Ford I, Ferrari R. Ivabradine in stable coronary artery disease. N Engl J Med 2014; 371: 2435.

248. Dhule SS, Gawali SR. Platelet aggregation and clotting time in type II diabetic males. Natl J Physiol Pharm Pharmacol 2014; 4: 121-123.

249. Güven F, Yilmaz A, Aydin H, Korkmaz I. Platelet aggregation responses in type 2 diabetic patients. Health 2010;2: 708-712.

250. Vinik A. Diabetic neuropathy: pathogenesis and therapy. Am J Med 1999; 107: 17S-26S.

251. Jordan J, Shannon JR, Black BK, Ali Y, Farley M, Costa F, Diedrich A, Robertson RM, Biaggioni I, Robertson D. The pressor response to water drinking in humans: a sympathetic reflex? Circulation 2000; 101: 504-509.

252. Singer W, Opfer-Gehrking TL, McPhee BR, Hilz MJ, Bharucha AE, Low PA. Acetylcholinesterase inhibition: a novel approach in the treatment of neurogenic orthostatic hypotension. J Neurol Neurosurg Psychiatry 2003; 74: 1294-1298. 
253. Nobrega AC, dos Reis AF, Moraes RS, Bastos BG, Ferlin EL, Ribeiro JP. Enhancement of heart rate variability by cholinergic stimulation with pyridostigmine in healthy subjects. Clin Auton Res 2001; 11: 11-17. 


\section{ABBREVIATIONS LIST}

ABPM

ACCORD

Diabetes trial

ACE

ADA ambulatory blood pressure monitoring

the Action to Control Cardiovascular Risk in

angiotensin-converting enzyme

American Diabetes Association

ADDITION the Anglo-Danish-Dutch Study of Intensive Treatment in People with Screen Detected Diabetes in Primary Care

AGEs

ALA

AMP

AngII

ANS

$\mathrm{AO}$

ApoA1

ApoB

ARB

ARIs

ASA

ASO advanced glucose end-products

alpha-lipoic acid

adenosine monophosphate

angiotensin II

autonomic nervous system antioxidant

apolipoprotein A1

apolipoprotein B

angiotensin II type 1 receptor blocker

aldose reductase inhibitors

acetylsalicylic acid

antisense oligonucleotide

ASCVD the aggressive lipid lowering on the primary prevention of atherosclerotic cardiovascular disease

ATR

BFT

BMI

BP

BRS

CAD

CAN

CARDs

CARTs

cGMP

CHD

$\mathrm{CoQ}_{10}$

CVD

DCCT

DGAT-2

DGLA

DHLA

DHPG

DIAD the Detection of Ischemia in Asymptomatic Diabetic Subjects study

DLP

DM

DNA

DOPA

DPN

DPP

adenosine triphosphate

benfotiamine

body mass index

blood pressure

baroreflex sensitivity

coronary artery disease

cardiac autonomic neuropathy

the Collaborative Atorvastatin Diabetes Study

cardiovascular autonomic reflex tests

cyclic guanosine monophosphate

coronary heart disease

coenzyme Q10

cardiovascular disease

the Diabetes Control and Complications Trial

diacylglycerol acyltransferase-2

dihomo- $\gamma$-linolenic acid

dihydrolipoic acid

dihydroxyphenylglycol

dyslipidemia

diabetes mellitus

deoxyribonucleic acid

dihydroxyphenylalanine

diabetic polyneuropathy

diabetic prevention program 
DSPN diabetic simmetrial sensory neuropathy

ECG electrocardiography

EDIC the Epidemiology of Diabetes Interventions and Complications study

EF

eNOS

ejection fraction

EURODIAB

Diabetes study

FDA endothelial NO synthase 3

the European Epidemiology and Prevention of

FIELD the Fenofibrate Intervention and Event Lowering in Diabetes study

GLUT4

GPx

GSH

GSR

HbAlc

HDL-C

HED

$\mathrm{HF}$

variability

HMG-CoA

$\mathrm{H}_{2} \mathrm{O}_{2}$

$\mathrm{HPS}_{2}$

HR

HRT

HRV

hs-CRP

IDDM

IL

IR

IRI

6-keto-PG F1 alpha

LA

LDL-C

LDLR

LF

variability

LPO

LV

MDA

MetS

MI

MIBG

MNSI

MSNA

MTP

$\mathrm{NADH}$

$\mathrm{NADPH}$

$\mathrm{NCV}$ the Food and Drug Administration

glucose transporter type 4

glutathione peroxidase

glutathione

glutathione reductase

glycated hemoglobin

high-density lipoprotein cholesterol

[11C]-meta-hydroxyephedrine

the high-frequency component of the heart rate

hydroxy-3-methylglutaryl-coenzymeA

hydrogen peroxide

the Heart Protection Study

heart rate

heart rate turbulence

heart rate variability

high-sensitivity C-reactive protein

Insulin-dependent diabetes mellitus

interleukin

insulin resistance

immunoreactive insulin

lipoic acid

6-keto-prostaglandin F1 alpha

low-density lipoprotein cholesterol

low density lipoprotein receptor

the low-frequency component of the heart rate

lipid peroxidation

left ventricular

malondialdegide

metabolic syndrome

myocardial infarction

[123I]-meta-iodobenzylguanidine

the Michigan Neuropathy Screening Instrument

muscle sympathetic nerve activity microsomal TG transfer protein

nicotinamide adenine dinucleotide

nicotinamide adenine dinucleotide phosphate

nerve conduction velocity 
NF- $\mathrm{BB}$ p65

$\mathrm{NO}$

Nox

NPC1L1

$\mathrm{OH}$

OS

PCSK9

PDE5

PKC intervals differ by more than $50 \mathrm{~ms}$ over $24 \mathrm{~h}$

PNP

POTS

PPAR- $\alpha$

PUFAs

QRS-T angle

QTd

QTi

RAGE

RMSSD intervals

RNS

ROS

SDANN

HR intervals

SMI

SOD Diabetes and Microalbuminuria trial

TD

T1DM

T2DM

TGs

TNF- $\alpha$

TxB2

TZDs

UKPDS

VADT

VDT

VLDL-C

VLF

variability
pNN50 the number of instances per hour in which two consecutive HR

Steno the Intensified Multifactorial Intervention in Patients With Type 2

nuclear transcription factor p65

nitric oxide

NADPH oxidase

Niemann-Pick C1-like protein 1

orthostatic hypotension

oxidative stress

proprotein convertase subtilisin/kexin type 9

phosphodiesterase-5

protein kinase $\mathrm{C}$

peripheral neuropathy

postural tachycardia syndrome

peroxisome proliferator-activated receptor- $\alpha$

polyunsaturated fatty acids

spatial QRS-T angle

QT dispersion

QTc interval

receptor for advanced glucose end-products

the root-mean square of the difference of successive R-R

reactive nitrogen species

reactive oxygen species

the standard deviation of 5-min average of normal

"silent" myocardial ischemia

superoxide dismutase

thiamine deficiency

type 1 diabetes mellitus

type 2 diabetes mellitus

triglycerides

tumour necrosis factor- $\alpha$

thromboxane B2

thiazolidinediones

the United Kingdom Prospective Diabetes Study

the Veteran Affairs Diabetes Trial

vibration detection threshold

very-low-density lipoprotein cholesterol

the very low frequency component of the heart rate 
Victoria Serhiyenko, Alexandr Serhiyenko

\title{
Diabetic cardiovascular neuropathy
}

\author{
Logos Publisher \\ Web-site: http//центр-логос.pф \\ E-mail: logos.centr@mail.ru
}

Подписано в печать 20.01.2018. Формат 60x84 1/16 Бумага офсетная. Гарнитура «Тіmes». Печать цифровая.

Усл. печ. л. 6,45 Заказ № 1091. Тираж 500 экз.

Отпечатано с готового оригинал-макета в типографии издательскополиграфического комплекса СКФУ, г. Ставрополь, пр. Кулакова, 2 